\author{
Aus der Klinik für Ophthalmologie \\ (Direktor: Prof. Dr. J. Roider) \\ im Universitätsklinikum Schleswig-Holstein, Campus Kiel \\ an der Christian-Albrechts-Universität zu Kiel
}

\title{
GLAUKOMSCREENING \\ MIT DEM BLAULICHT-MUSTER-ERG UND DEM OCT - SIND BEIDE VERFAHREN GLEICHWERTIG?
}

\author{
Inauguraldissertation \\ zur \\ Erlangung der Doktorwürde \\ der Medizinischen Fakultät \\ der Christian-Albrechts-Universität zu Kiel
}

vorgelegt von

KATRIN, WOLFF

aus Teterow

Kiel 2013 
1. Berichterstatter:

2. Berichterstatter:

Tag der mündlichen Prüfung:

Zum Druck genehmigt, Kiel, den

gez.
Prof. Dr. J. Roider

Prof. Dr. P. Ambrosch

08.01.2014

08.01 .2014

Prof. Dr. J. Hillenkamp 


\section{Inhaltsverzeichnis}

Verzeichnis der Abkürzungen

Verzeichnis der Abbildungen

Verzeichnis der Tabellen

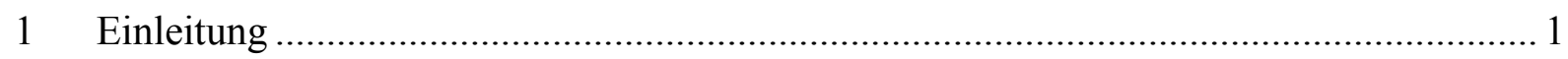

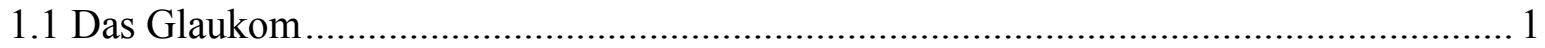

1.2 Die Optische Kohärenztomographie (OCT) ........................................................ 2

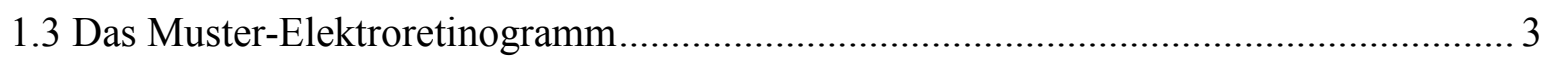

1.4 Der Blausinn und die Hypothese der ,,reduzierten Redundanz“ .................................... 4

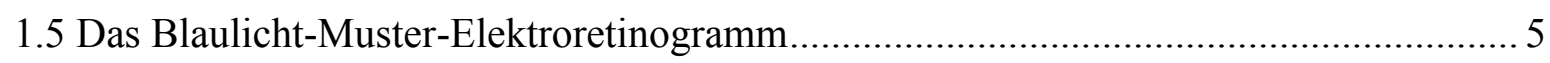

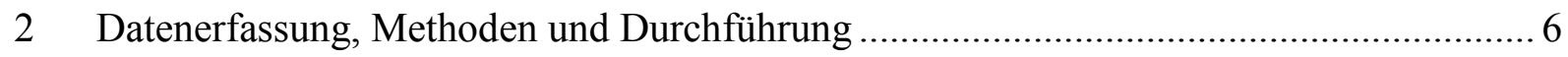

2.1 Ein- und Ausschlusskriterien zur Auswahl der Probanden und Patienten ...................... 6

2.2 Konformität mit der Deklaration des Weltärztebundes von Helsinki/Tokio (1964/1975)

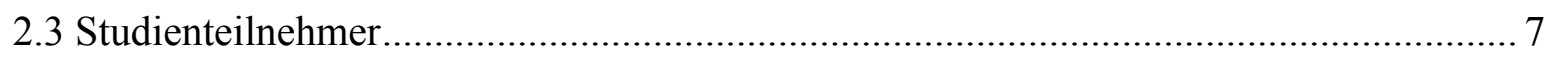

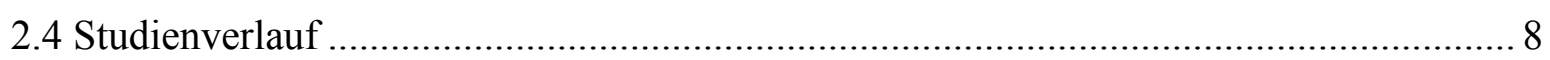

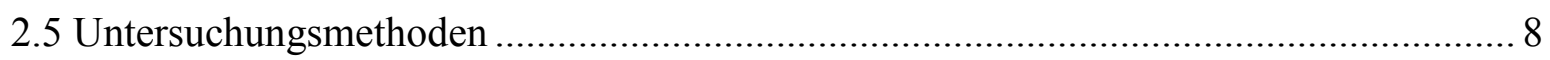

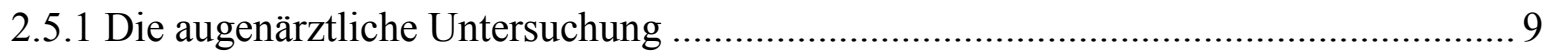

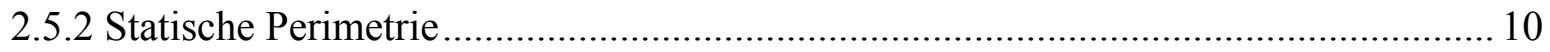

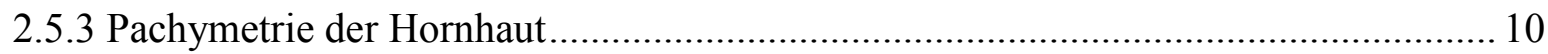

2.5.4 Nervenfaserschichtdickenmessung mittels optischer Kohärenztomographie (OCT) . 11

2.5.5 Muster-Elektroretinogramm (= Pattern-ERG = P-ERG) ...................................... 11

2.5.6 Blaulicht-Muster-Elektroretinogramm (= Blaulicht-P-ERG) .................................. 13

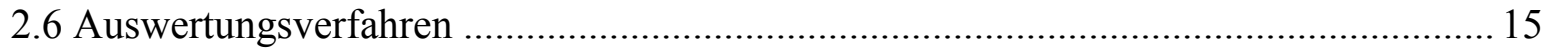

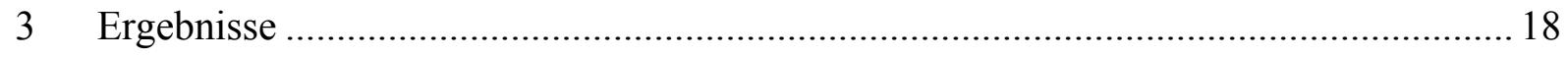

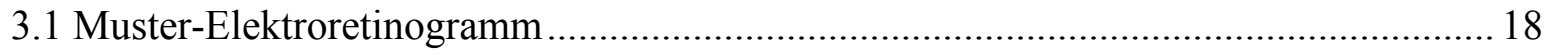

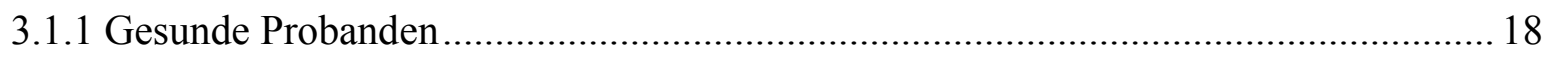

3.1.2 Alterskorrelierte Verteilung des Blaulicht-Muster-ERGs ...................................... 18

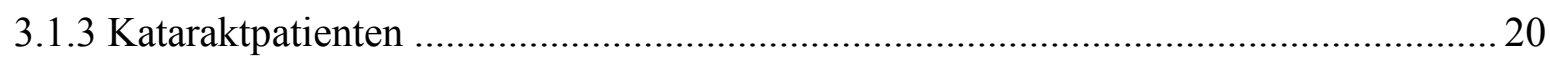

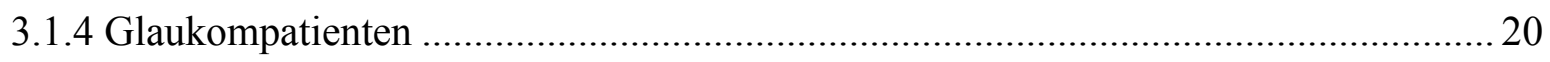

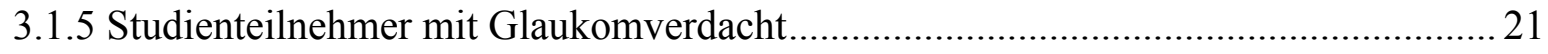




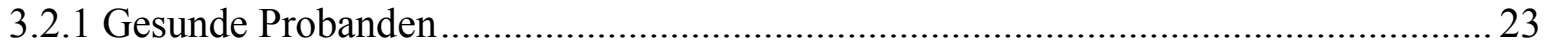

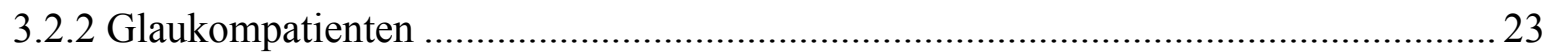

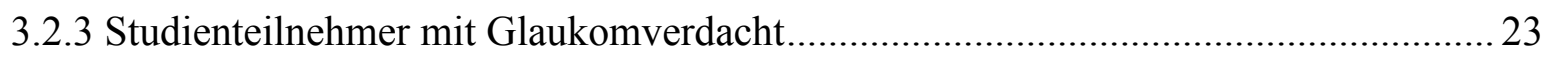

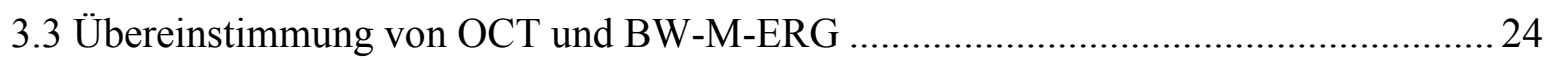

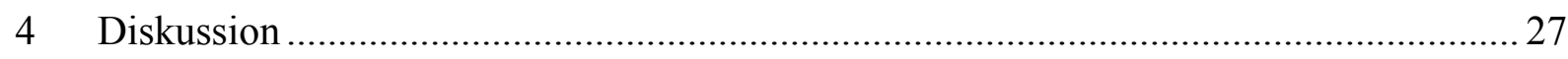

4.1 Das Muster-Elektroretinogramm heute in der medizinischen Anwendung .................. 27

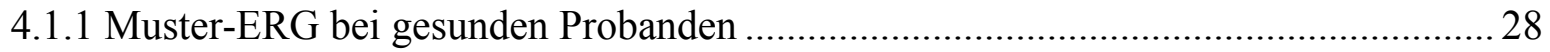

4.1.2 Alterskorrelierte Verteilung des Blaulicht-Muster-ERGs ......................................... 28

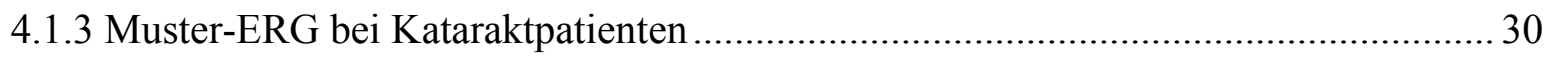

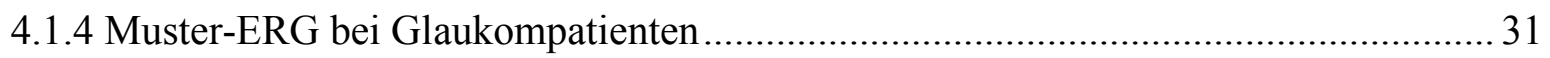

4.1.5 Muster-ERG bei Studienteilnehmern mit Glaukomverdacht ........................................ 32

4.2 Die optische Kohärenztomographie heute in der medizinischen Anwendung .............. 33

4.2.1 Nervenfaserschichtdickenmessung mittels Stratus OCT bei gesunden Probanden .... 33

4.2.2 Nervenfaserschichtdickenmessung mittels Stratus OCT bei Glaukompatienten ........ 35

4.2.3 Nervenfaserschichtdickenmessung mittels Stratus OCT bei Studienteilnehmern mit

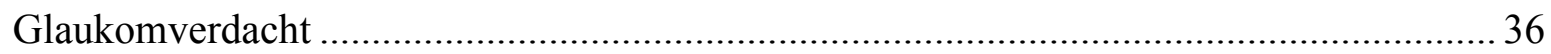

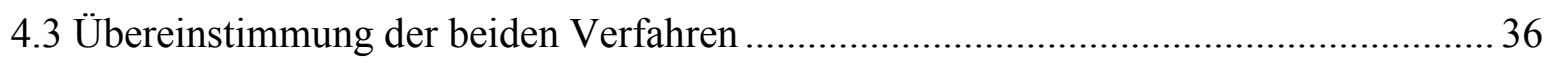

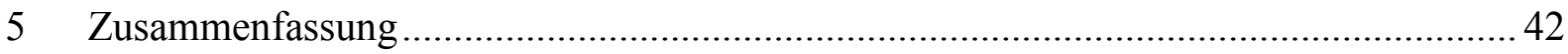

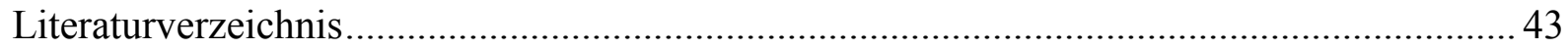

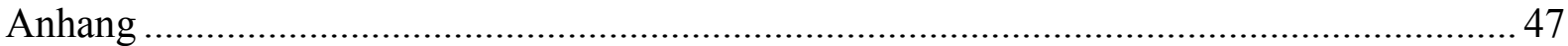

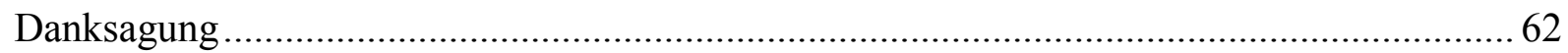

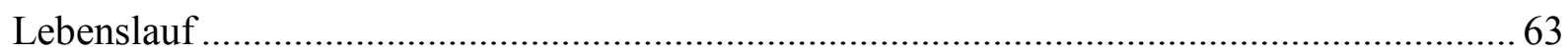




\section{Verzeichnis der Abkürzungen}

\begin{tabular}{|c|c|}
\hline AMD & altersbedingte Makuladegeneration \\
\hline $\mathrm{BH}$ & Bindehaut \\
\hline BW-M-ERG & Blau-zu- Weiß-Muster-Elektroretinogramm \\
\hline Cat. incip. & Cataracta incipiens (= beginnende Linsentrübung) \\
\hline CDR & Cup-/Disc-Ratio \\
\hline CL & Kontaktlinsen \\
\hline $\csc$ & cum sua correctione (= mit eigener [Brillen-] Korrektion) \\
\hline ED & Erstdiagnose \\
\hline ERG & Elektroretinogramm \\
\hline et al. & et alii (= und andere) \\
\hline GF & Gesichtsfeld \\
\hline $\mathrm{HH}$ & Hornhaut \\
\hline ID & Identifikationsnummer \\
\hline inf. & inferior \\
\hline IOD & intraokularer Druck \\
\hline ISNT & inferior-superior-nasal-temporal \\
\hline$\kappa$ & Cohen’s Kappa (= Übereinstimmungsmaß) \\
\hline KT und RT & Kern- und Rindentrübung \\
\hline LB & Lesebrille \\
\hline Mac. & Makula \\
\hline Max & Maximum \\
\hline MD-D und MD-S & Mean deviation dexter und sinister \\
\hline & (= Mittlere Abweichung rechts und links) \\
\hline M-ERG (= P-ERG) & Muster-Elektroretinogramm (= Pattern-Electroretinogramm) \\
\hline mfERG & multifokales Elektroretinogramm \\
\hline Min & Minimum \\
\hline MW & Mittelwert \\
\hline N35 & negativer Ausschlag nach 35 ms im ERG \\
\hline N95 & negativer Ausschlag nach $95 \mathrm{~ms}$ im ERG \\
\hline nas. & nasal \\
\hline OCT & optische Kohärenztomographie \\
\hline
\end{tabular}


OD

Oculus dexter (= rechtes Auge)

OS

Oculus sinister (= linkes Auge)

P50

positiver Ausschlag nach $50 \mathrm{~ms}$ im ERG

PEV

Pigmentepithelverschiebung

POWG (= OAG) primäres Offenwinkelglaukom (= Open-Angle-Glaucoma)

PSD-D und PSD-S Pattern standard deviation dexter und sinister

(= Homogenitätsabweichung rechts und links)

RNFL retinal nerve fibre layer (= retinale Nervenfaserschichtdicke)

rs

randscharf

s. c.

sine sua correctione (= ohne [Brillen-]Korrektur)

SD

Standardabweichung

SD-OCT (= HD OCT) Spektral Domänen-OCT (= high definition OCT)

SSNE

schräger Sehnerveneintritt

S-Typ

Short-Typ (= kurzwelliger Zapfentyp)

sup.

superior

SW

Spannweite

TD-OCT

Time domain-OCT $(\triangleq$ Stratus OCT $)$

temp.

temporal

Th. lt. Brief

Therapie laut Brief 


\section{Verzeichnis der Abbildungen}

Abb. 1: $\quad$ Ausdruck einer Nervenfaserschichtdickenmessung mittels Stratus OCT

Abb. 2: $\quad$ Elektrodenanordnung am Studienteilnehmer

Abb. 3: Goldfolienelektrode im linken Bindehautsack und Referenzelektrode an der linken Schläfe

Abb. 4: $\quad$ Normales Muster-ERG mit Darstellung der N95-Amplitudenmessung [modifiziert aus 26]

Abb. 5: $\quad$ Proband zur Messung des Blaulicht-Muster-ERGs

Abb. 6: Transmissiononsspektrum des verwendeten optischen Filters

Abb. 7: $\quad$ Absorptionsspektrum der drei Farbrezeptoren und der Zapfen [35]

Abb. 8: $\quad$ Berechnung der durchschnittlichen Nervenfaserschichtdicke mittels Stratus OCT

Abb. 9: N95-Amplitude eines Weiß-Muster-ERGs bei einem gesunden Probanden

Abb. 10: N95-Amplitude eines Blaulicht-Muster-ERGs bei einem gesunden Probanden

Abb. 11: P-ERG-Messungen in Abhängigkeit vom Alter bei Normalprobanden

Abb. 12: Alterskorrigierte Eichkurve für die Katarakt erstellt an Normalprobanden

Abb. 13: P-ERG-Messungen in Abhängigkeit vom Alter bei Glaukompatienten

Abb. 14: P-ERG-Messungen in Abhängigkeit vom Alter bei „eher glaukommanifesten“ Glaukomverdachtspatienten

Abb. 15: P-ERG-Messungen in Abhängigkeit vom Alter bei „eher gesunden“ Glaukomverdachtspatienten 


\section{Verzeichnis der Tabellen}

Tabelle 1: $\quad$ Gruppe I (gesunde Probanden)

Tabelle 2: $\quad$ Gruppe II (Kataraktpatienten)

Tabelle 3: Gruppe III (Glaukompatienten)

Tabelle 4: Gruppe IV (Verdachtspatienten)

Tabelle 5: $\quad$ Studienteilnehmer

Tabelle 6: P-ERG- und OCT-Ergebnisse der Gruppe I (gesunde Probanden)

Tabelle 7: Alterskorrelierte Blau- zu Weiß-Muster-ERG-Normwerte

Tabelle 8: $\quad$ P-ERG- und OCT-Ergebnisse der Gruppe II (Kataraktpatienten)

Tabelle 9: $\quad$ P-ERG- und OCT-Ergebnisse der Gruppe III (Glaukompatienten)

Tabelle 10: P-ERG- und OCT-Ergebnisse der Gruppe IV (Verdachtspatienten)

Tabelle 11: RNFL-Ergebnisse der Normalprobanden

Tabelle 12: RNFL-Ergebnisse der Glaukompatienten

Tabelle 13: RNFL-Ergebnisse der Glaukomverdachtspatienten

Tabelle 14: Häufigkeitsverteilung der Studienteilnehmer in der Endauswertung

Tabelle 15: Kreuztabelle von retinaler Nervenfaserschichtdickenmessung und Blau- zu Weiß-Muster-ERG

Tabelle 16: Häufigkeitsverteilung der 72 gesunden Studienteilnehmer gemäß RNFLMessung

Tabelle 17: Häufigkeitsverteilung der 10 erkrankten Studienteilnehmer gemäß RNFLMessung

Tabelle 18: Häufigkeitsverteilung der 66 gesunden Studienteilnehmer gemäß beiden Messungen

Tabelle 19: Häufigkeitsverteilung der 71 gesunden Studienteilnehmer gemäß BWERGMessung

Tabelle 20: Häufigkeitsverteilung der 11 erkrankten Studienteilnehmer gemäß BWERGMessung

Tabelle 21: Häufigkeitsverteilung der 5 erkrankten Studienteilnehmer gemäß beiden Messungen

Tabelle 22: ERG- und RNFL-Ergebnisse im Vergleich zur Realität

Tabelle 23: Cohen's Kappa-Koeffizient $\kappa$

Tabelle 24: Bewertung von $\kappa[39]$ 


\section{Einleitung}

\section{$1 \quad$ Einleitung}

\subsection{Das Glaukom}

Der Begriff „Glaukom“ leitet sich vom griechischen $\gamma \lambda \alpha v \kappa o ́ \varsigma$ (glaukós) „hell“/,leuchtend“ [1] ab. Dies beschrieb die Farbe des Meeres „(grau)bläulich“ wie auch die Irisverfärbung bei chronischer Entzündung. Bis ins 18. Jahrhundert galt das Glaukom als Augenerkrankung, die ohne ersichtliche Ursache zur Sehverschlechterung oder Erblindung führte. Als Synonym etablierte sich im 20. Jahrhundert die Bezeichnung „Grüner Star“, wobei sich „grün“ wiederum von der Übersetzung aus dem Griechischen ableitete. Der Begriff „Star“ ist seit dem 8. Jahrhundert im Deutschen der Ausdruck für eine Linsentrübung. Häufig kam es zu Verwechslungen mit dem „Grauen Star“, der Katarakt. Erst mit Einführung der Kataraktoperation 1753 konnte gezeigt werden, dass nur ein Teil der Erblindungen durch Entfernung der Linse heilbar war, und man begann den grünen und grauen Star zu unterscheiden.

Als Glaukome bezeichnet man heute eine heterogene Gruppe von Augenerkrankungen, die zu einer progredienten Schädigung des Sehnerven mit Verlust visueller Funktion führen [2].

Bei der ophthalmologischen Untersuchung des Augenhintergrundes von Glaukompatienten manifestiert sich ein ausgehöhlter Sehnerv, die Papillenexkavation. Zur Beschreibung der Exkavation kann das Verhältnis der Aushöhlung zum gesamten Papillendurchmesser angegeben werden, die sogenannte Cup-/Disc-Ratio (CDR). Die meisten Menschen haben eine CDR von 0,3 oder weniger [3]. Als sicher glaukomverdächtig gilt eine CDR größer 0,7 [4].

Die glaukomspezifischen Gesichtsfeldeinschränkungen sind Folge der kontinuierlichen Sehnervenschädigung. Mittels Perimeter können diese Ausfälle gemessen werden. Charakteristische Gesichtsfelddefekte sind die sogenannten Bjerrum-Skotome, die vom blinden Fleck ausgehen und bogenförmig in der mittleren Peripherie gelegen sind [3]. Allerdings werden sie meist erst signifikant bei einem bestehenden Nervenzellverlust von mehr als 60\% [5]. Dadurch ist der Begriff des ,präperimetrischen Glaukoms“ entstanden. Dieser beschreibt die Zeit, in der die Erkrankung bereits besteht, jedoch in der zur Diagnostik eingesetzten Gesichtsfelduntersuchung noch nicht auffällt und ist somit insbesondere Ziel von Screening-Tests.

Ein wichtiger Faktor für die Entwicklung von Glaukomschäden ist der Augeninnendruck. Der normale intraokulare Druck (IOD) beträgt 10 bis $21 \mathrm{mmHg}$ und wird beispielsweise mit dem Goldmann-Applanationstonometer gemessen. Werte von mehr als $21 \mathrm{mmHg}$, okuläre 


\section{Einleitung}

Hypertensionen, sind mit einem erhöhten Risiko für die Entwicklung des Glaukoms assoziiert [6]. Der IOD wird aufrecht erhalten durch eine ständige Kammerwasserproduktion und dem geregelten Kammerwasserabfluss. Beim Glaukom entsteht die Steigerung des Augeninnendrucks nicht durch eine Kammerwasserüberproduktion, sondern ausschließlich durch eine Abflussbehinderung.

Die mit Abstand häufigste Glaukomform ist das primär chronische Offenwinkelglaukom (POWG), bei dem der Kammerwinkel durch Ablagerung hyalinen Materials verlegt ist [7; 8]. $\mathrm{Ab}$ dem 40. Lebensjahr beträgt die Prävalenz des POWGs in Europa etwa 2\% und steigt mit dem Alter an [9;10]. Nach Studien von Kass et al. konvertieren 9,5\% der Patienten mit okulärer Hypertension binnen 5 Jahren zum POWG [11]. Allerdings weisen etwa ein Drittel aller Patienten mit einem Offenwinkelglaukom Drücke unter $21 \mathrm{mmHg}$ auf und müssen somit als Normaldruckglaukom klassifiziert werden [9]. Dies bedeutet, dass hier bereits Drücke im Normbereich zu einer Sehnervenschädigung geführt haben. Es ist vorwiegend eine Erkrankung des älteren Menschen und häufiger bei Frauen. Es tritt vermehrt in Kombination mit kardiovaskulären Risikofaktoren, wie beispielsweise Blutdruckschwankungen [3] auf. So beschreiben auch Plange et al., dass vor allem nächtliche Hypotonien durch den relativ zum Perfusionsdruck zu hohen Augeninnendruck zu einer Sehnervenminderperfusion führen [12]. Diese Erkenntnisse verdeutlichen, dass die alleinige IOD-Messung nicht für ScreeningUntersuchungen geeignet ist.

Das Glaukom ist nach der altersbedingten Makuladegeneration (AMD) und der diabetischen Retinopathie die häufigste Erblindungsursache in den Industrienationen. In Deutschland ist es nach der AMD der zweithäufigste Grund für Blindengeldbezug [13; 14].

Auch aus diesem Grund wäre ein Screening der Bevölkerung zum frühzeitigen Erkennen und Behandeln des Glaukoms sozioökonomisch sinnvoll und wünschenswert, jedoch existiert zur Zeit noch kein Verfahren, welches eine angemessene Sensitivität sowie Spezifität garantiert.

\subsection{Die Optische Kohärenztomographie (OCT)}

Die Möglichkeiten der Bildgebung in der Augenheilkunde sind in den letzen 20 Jahren enorm gewachsen. Die Pentacam mit Scheimpflug-Kamera, Lasersysteme mit Wellenfrontanalysegeräten und die optische Kohärenztomographie sind nur einige der Entwicklungen. Anfang der 90iger Jahre erschienen die ersten Artikel über die optische Kohärenztomographie [15; 16]. Im Jahre 1994 patentierte die Firma Carl Zeiss Meditec, Inc. Dublin, California, die Technologie der OCT. 1995 bezeichneten Schuman et al. das OCT als 


\section{Einleitung}

neues Verfahren in der Glaukomdiagnostik [17]. Das erste kommerziell erhältliche OCTGerät kam 1996 auf den Markt. Vier Jahre später erschien das OCT 2000 und nach weiteren zwei Jahren das Stratus OCT (OCT3), welches heute den „Goldstandard“ der Netzhautbildgebung darstellt. Das zur Zeit aktuellste Modell ist seit 2006 das hochauflösende, schnell rasterscannende (HD-)OCT [18].

Die gesamte Technologie beruht auf einem ähnlichen Prinzip wie die Sonographie, mit dem Unterschied, dass hierbei nicht Schall, sondern Licht geringer Kohärenzlänge verwendet wird. In einem Rasterverfahren rund um den Sehnervenkopf wird mit einem Lichtstrahl die Netzhaut abgetastet. Das Untersuchungssignal wird reflektiert und mit einem Referenzsignal überlagert. Aus dem sich ergebenden Interferenzmuster lässt sich die Entfernung messen, ein Tiefenprofil erstellen und ein flächiges Bild oder ein dreidimensionales Volumen rekonstruieren. In der Augenheilkunde findet das OCT vorrangig Anwendung bei der Diagnostik von Makulopathien sowie bei der Beurteilung von Nervenfaserschichten und des Sehnervenkopfes.

Die optische Kohärenztomographie ist ein berührungsfreies, nicht invasives Verfahren, das leicht und schnell durchführbar ist. Es stellt geringe Anforderungen an die Patientencompliance und bietet dennoch wichtige Informationen über die peripapilläre Netzhautdicke, die bedeutende Hinweise in der Glaukomdiagnostik sind. So beschrieb beispielsweise Gyatsho et al. eine Ausdünnung der Nervenfaserschicht bei Glaukompatienten, die mittels OCT detektiert werden konnte [19].

\subsection{Das Muster-Elektroretinogramm}

Das Muster-Elektroretinogramm (engl.: pattern-ERG, daher: P-ERG) ist ein elektrophysiologisches Verfahren, bei dem vorrangig die Ganglienzellfunktion der Retina überprüft wird. 1982 beschrieben May et al. das erste auffällige P-ERG eines Patienten mit klinisch signifikantem Sehnervenschaden [20]. 1984 folgte ein Bericht von Papst et al. über die ersten pathologischen Muster-ERGs bei Glaukompatienten [21]. Die Gruppe von Arden et al. postulierten 1988 bereits die Entdeckung des ,präperimetrischen Glaukoms“ durch das P-ERG [22]. Heute beweisen diverse Studien, dass der retinale Ganglienzelluntergang sehr gut mittels Muster-ERG dargestellt werden kann [23; 24; 25].

Die Untersuchung wird standardisiert durchgeführt gemäß den Richtlinien der International Society for Clinical Electrophysiology of Vision (ISCEV) [26]. Mit Hilfe eines alternierenden Schachbrettmusters wird mit weißen und schwarzen Flächen bei konstanten 


\section{Einleitung}

Lichtverhältnissen dem menschlichen Auge auf einem Computermonitor ein ständig wechselnder Musterreiz dargeboten. Die gemessenen Reizantworten werden über Elektroden neben dem Auge sowie über eine Hornhautelektrode abgeleitet. Da die Potentiale im $\mu \mathrm{V}$ Bereich liegen, werden jeweils Summenpotentiale aus 100 bis 150 Messungen gemittelt [27]. Dennoch führt jegliche Beeinträchtigung der retinalen Bilddarstellung zu einer deutlichen Reduktion der Amplituden im Muster-ERG [28]. Die wichtigste Vorraussetzung ist der ungetrübte und bestmögliche Visus des Patienten. Testpersonen mit fortgeschrittener Linsentrübung, einer Sehschärfe kleiner 50\% oder sonstigen Visusminderungen werden per se nur noch geringe bis gar keine Reizantworten darbieten.

\subsection{Der Blausinn und die Hypothese der „reduzierten Redundanz“}

Für das menschliche Auge ist Licht im Wellenlängenbereich von 390 bis etwa $700 \mathrm{~nm}$ sichtbar. Es existieren zwei unterschiedliche Systeme von visuellen Rezeptoren: Stäbchen zur Hell-Dunkelwahrnehmung und Zapfen zur Farbdifferenzierung, wobei man diese unterteilt in jene mit einem Absorptionsmaximum im kurz- (S-Typ), mittel- (M-Typ) und langwelligen Bereich (L-Typ). Der S-Typ hat sein Absorptionsmaximum bei etwa $420 \mathrm{~nm}$ und ist für blaues Licht empfindlich, den Blausinn. Die durch Licht der Wellenlänge von etwa 390 - 500 nm ausgelösten Aktionspotentiale werden hauptsächlich von den Blauzapfen über das koniozelluläre Ganglienzellsystem in die interlaminären Schichten des Corpus geniculatum laterale geleitet [29]. Diese Ganglienzellgruppe hat einen Anteil von 10\% aller retinalen Ganglienzellen und ist ausschließlich mit S-Zapfen verbunden, die wiederum ebenfalls nur einen 10tel aller Zapfen darstellen [30]. Demzufolge ist das koniozelluläre Ganglienzellsystem hoch spezialisiert und wird im Rahmen der Hypothese der „reduzierten Redundanz“ für die Diagnostik interessant. Der Hintergrund dieser Annahme ist, dass man davon ausgeht, dass kleine Zellpopulationen bei Störungen weniger in der Lage sind, Funktionsausfälle zu kompensieren [29]. Folglich zeigt dieses Defizit bei geeigneten Tests früher pathologische Veränderungen als es bei größeren Zellgruppen der Fall wäre.

Beim Glaukom wird von einem progredienten Schaden insbesondere der Ganglienzellen ausgegangen [19; 28]. Daher kann man mit einem selektiven Test einer kleinen Ganglienzellpopulation (wie den koniozellulären Ganglienzellen) bereits frühzeitig glaukomassoziierte Nervenschäden aufzeigen [31]. 


\section{Einleitung}

\subsection{Das Blaulicht-Muster-Elektroretinogramm}

Das Blaulicht-Muster-ERG ist eine im Rahmen dieser Studie von uns neu entwickelte Methode, die zur Glaukomdiagnostik beitragen soll. Die theoretische Grundlage dafür bildeten zwei Hypothesen der Glaukomfrüherkennung: Erstens werden retinale Ganglienzellen geschädigt, was zweitens besonders eindrücklich messbar ist bei kleinen Zellpopulationen wie den koniozellulären Ganglienzellen. Die Funktion der retinalen Ganglienzellen wird mittels Muster-Elektroretinogramm berechnet. Das koniozelluläre System kann durch kurzwelliges Licht stimuliert werden. Beides wird im Blaulicht-MusterERG kombiniert.

Es gilt zu überprüfen, ob die Kombination der vorstehend genannten Verfahren in der Glaukomfrühdiagnostik dem einzelnen Verfahren überlegen ist.

Ziel dieser Studie ist es, das Blaulicht-Muster-Eletroretinogramm auf Tauglichkeit zur Früherkennung zu überprüfen und es mit einem weitläufig angewandtem Verfahren in der Glaukomdiagnostik, der optischen Kohärenztomographie, zu vergleichen. 


\section{Datenerfassung, Methoden und Durchführung}

\section{Datenerfassung, Methoden und Durchführung}

Im Rahmen einer prospektiven Studie wird das neue Verfahren des Blaulicht-MusterElektroretinogramms in der Augenklinik des Universitätsklinikums Kiel an 30 gesunden Probanden auf Funktionsfähigkeit geprüft. Nach ersten konstruktiven Erkenntnissen erfolgt die Ausweitung der Versuchsreihen auf 20 Glaukomverdachtspatienten und 20 manifest Glaukomerkrankte, die entsprechend den Standardmaßnahmen beim Glaukom untersucht werden. Neben anderen Methoden wird insbesondere die übliche Nervenfaserschichtdickenmessung mittels optischer Kohärenztomographie (OCT) dem neuen Verfahren des Blaulicht-Muster-ERGs gegenübergestellt und auf Korrelationen getestet.

Ziel ist es zu erfahren, welche diagnostische Aussagekraft das Blau-P-ERG in Relation zum bereits in die Glaukomdiagnostik eingeführten OCT hat.

Des weiteren wird in einer kleinen Gruppe der Einfluss der Operation des grauen Stars auf die Ergebnisse der elektrophysiologischen Untersuchungen evaluiert. Erwartet werden höhere Potentiale im Elektroretinogramm nach Entfernung der Katarakt.

\subsection{Ein- und Ausschlusskriterien zur Auswahl der Probanden und Patienten}

Eingeschlossen wurden Teilnehmer, die älter als 18 Jahre waren, mindestens einen Fernvisus von 0,5 erreichten und eine weitgehend unauffällige Allgemeinanamnese angaben. Es wurden keine Patienten mit Diabetes mellitus in die Studie eingeschlossen, da Veränderungen der Farbwahrnehmung hier mehrfach nachgewiesen wurden [32;33]. Ein therapeutisch gut eingestellter arterieller Hypertonus war kein Ausschlusskriterium.

\subsection{Konformität mit der Deklaration des Weltärztebundes von Helsinki/Tokio} (1964/1975)

Alle Probanden und Patienten waren über die Natur der Studie, ihren Zweck und mögliche Risiken und Komplikationen in allen Einzelheiten informiert und gaben schriftlich ihr Einverständnis zu den Untersuchungen. Die Ethik-Kommission der Medizinischen Fakultät der Christian-Albrechts-Universität zu Kiel genehmigte diese Untersuchungen. 


\section{Datenerfassung, Methoden und Durchführung}

\subsection{Studienteilnehmer}

30 gesunde Studenten und Mitarbeiter der Universitäts-Augenklinik, 6 Männer und 24 Frauen, bilden die Gruppe der Normalprobanden, die Gruppe I (Tabelle 1 im Anhang). Sie sind im Durchschnitt 34 Jahre (21-60 Jahre) alt und weisen weder bei der Gesichtsfelduntersuchung Skotome noch bei den ophthalmologischen Untersuchungen hypertone intraokulare Drücke (IOD) oder glaukomverdächtige Papillen auf. Von diesen 30 Probanden sind 13 emmetrop. Die verbleibenden 17 nehmen mit bestkorrigierenden Brillengläsern an der Studie teil.

Als Nebenarm der Studie werden in Gruppe II (Tabelle 2 im Anhang) vier Patienten von durchschnittlich 63,5 Jahren mit bekanntem grauen Star mittels Standard- und BlaulichtMuster-ERG untersucht. Zwei der Patienten sind gesund, während die anderen zwei bekanntermaßen am Glaukom erkrankt sind.

Die Glaukompatienten, Gruppe III (Tabelle 3 im Anhang), werden rekrutiert aus Patienten, die zur Erstellung eines Tagesdruckprofils in der Universitäts-Augenklinik Kiel stationär aufgenommen werden. Es werden von den Patienten in dieser Gruppe in mindestens zwei von drei Punkten die klassischen Glaukomkriterien gemäß den Leitlinien des Berufsverbands der Augenärzte Deutschlands und der Deutschen Ophthalmologischen Gesellschaft [13; 14] erfüllt:

1) typischer Gesichtsfeldausfall (13/20);

2) Druckerhöhung zeitweise über $21 \mathrm{mmHg}(2 / 20)$;

3) glaukomtypischer Sehnervenschaden (20/20).

$\mathrm{Zu}$ den glaukomtypischen Sehnervenschäden gehören eine vertikale C/D-Ratio der Papille größer als 0,6 (unter Berücksichtigung der Papillengröße), eine diffuse oder fokale Verdünnung der neuroretinalen Randzone (insbesondere am unteren oder oberen Pol, ISNTRegel), Randblutungen der Papille, eine Seiten-Asymmetrie glaukomtypischer Papillenveränderungen zwischen beiden Augen, diffuse/fokale Ausfälle der Nervenfaserschicht (z.B. Defekte der retinalen Nervenfaserschicht) bei Untersuchung im rotfreiem Licht sowie eine bajonettförmige Abknickung der Gefäße am Papillenrand [14].

Von den 20 Glaukompatienten sind acht männlich und zwölf weiblich bei durchschnittlich 66 Jahren (48-83 Jahre). Es wurden nur Glaukompatienten mit einem gonioskopisch offenen 


\section{Datenerfassung, Methoden und Durchführung}

Kammerwinkel in diese Studie einbezogen. Bei 2 der 20 Patienten lag ein Normaldruckglaukom vor. Da die Diagnose des Glaukoms bei diesen Patienten eindeutig belegt ist, sind alle mit einer augeninnendrucksenkenden Therapie eingestellt.

Die 20 Teilnehmer mit Glaukomverdacht, die Gruppe IV (Tabelle 4 im Anhang), sind ebenfalls Patienten, die zur Glaukomdiagnostik in die Augenklinik kamen. Das Durchschnittsalter beträgt 43 Jahre (18-69 Jahre). Es nehmen neun Männer und elf Frauen teil. Der Glaukomverdacht wird gestellt durch das Vorhandensein mindestens eines der Kriterien Gesichtsfeldeinschränkungen (6/20), erhöhter Augeninnendruck (3/20) oder auffällige Papillenexkavationen (15/20). Da es sich bei diesen Patienten um einen Glaukomverdacht handelt, bekommen die meisten Patienten keine ophthalmologische Medikation. Sechs Patienten erhielten jedoch vorsorglich antiglaukomatöse Augentropfen.

Tabelle 5: Studienteilnehmer

\begin{tabular}{|l|c|c|c|}
\hline & $\mathrm{n}=$ & $\mathrm{M}: \mathrm{F}$ & Alter \\
\hline I) gesunde Probanden & 30 & $6: 24$ & 33,7 \\
II) Kataraktpatienten & 4 & $0: 4$ & 63,5 \\
III) Glaukompatienten & 20 & $8: 12$ & 65,9 \\
IV) Verdachtspatienten & 20 & $9: 11$ & 43,0 \\
\hline
\end{tabular}

\subsection{Studienverlauf}

$\mathrm{Zu}$ Beginn werden hauptsächlich gesunde Probanden mittels Standard- und Blaulicht-MusterERG untersucht, um zu prüfen, ob das neue Verfahren plausible Ergebnisse liefert. Nach ersten entsprechenden Erkenntnissen werden weitere diagnostische Verfahren ergänzt.

\subsection{Untersuchungsmethoden}

30 Normalprobanden und je 20 Glaukom- sowie Glaukomverdachtspatienten durchlaufen in dieser Studie die Basisdiagnostik des Glaukoms, wozu folgende Verfahren gehören [13; 14]:

1. die Erhebung einer augenärztlichen Anamnese und eingehende Untersuchung

2. eine Gesichtsfeldmessung mit dem Perimeter (30-2er Programm des Humphrey Projektionsperimeter HFA II) 


\section{Datenerfassung, Methoden und Durchführung}

3. Pachymetrie der Hornhaut

4. eine Messung der Nervenfaserschichtdicke mit dem OCT (Stratus OCT Version 4.0).

Zusätzlich wird durchgeführt:

5. eine Testung der Netzhaut mit dem Muster-ERG

6. eine Überprüfung der Blauzapfenfunktion mit dem Blaulicht-Muster-ERG.

\subsubsection{Die augenärztliche Untersuchung}

Die augenärztliche Untersuchung erfolgt durch einen Arzt der Kieler Universitätsklinik für Augenheilkunde. Diese beinhaltet jeweils monokular eine:

- Refraktionsbestimmung

- Visusprüfung

- Spaltlampenuntersuchung

- Funduskopie

- Augeninnendruckmessung

Nach der subjektiven Refraktionsbestimmung wird der Fernvisus mittels Optotypen in $5 \mathrm{~m}$ Entfernung geprüft.

An der Spaltlampe wird der vordere Augenabschnitt und die Linse beurteilt. Patienten mit einer Visusminderung unter 0,5, einer starken Linsentrübung oder pathologischen Veränderungen des vorderen Augenabschnittes werden nicht für die Studie zugelassen.

Bei der Funduskopie wird die gesamte Netzhaut untersucht und der Untersucher beurteilt die Exkavation des Sehnervens.

Es wird weiterhin der Augeninnendruck mit Hilfe der Applanationstonometrie nach Goldmann gemessen. Hierbei wird nach Betäuben und Anfärben der Hornhautoberfläche mit Thilorbin ${ }^{\circledR}$-Augentropfen (Wirkstoffe: Oxybuprocain-HCl, Fluorescein-Natrium) die Kraft gemessen, die für eine definierte mechanische Abplattung der Hornhaut erforderlich ist. Eine Augeninnendruckerhöhung ist ab einem intraokularen Druck von $22 \mathrm{mmHg}$ definiert.

Bei allen Patienten wird des weiteren eine Gonioskopie durchgeführt, wenn dies nicht schon im Rahmen von früheren Untersuchungen geschehen war. Dafür wird auf die ebenso betäubte Hornhaut ein Goldmann-Dreispiegelkontaktglas aufgesetzt. In dieses Kontaktglas sind drei 


\section{Datenerfassung, Methoden und Durchführung}

Spiegel mit unterschiedlichen Neigungswinkeln eingearbeitet. Jene mit einem Neigungswinkel von $66^{\circ}$ und $73^{\circ}$ dienen der Untersuchung der hinteren Augenabschnitte. Der dritte mit einem Neigungswinkel von $59^{\circ}$ erlaubt an der Spaltlampe einen spiegelbildlichen Einblick in den ansonsten nicht einsehbaren Kammerwinkel. Durch Drehen des Kontaktglases kann der Kammerwinkel zirkulär beurteilt werden. Damit wird ein Engwinkel- von einem Offenwinkelglaukom unterschieden, wobei für diese Studie nur Patienten mit einem Offenwinkelglaukom ausgewählt wurden.

\subsubsection{Statische Perimetrie}

Die Gesichtsfelduntersuchung erfolgt standardgemäß bei allen Kandidaten durch Schwellenwertperimetrie des zentralen $30^{\circ}$-Gebrauchsgesichtfeldes mit dem Projektionsperimeter HFA II der Firma Carl Zeiss Meditec, Jena. Dabei wird für jedes Auge getrennt die Sensitivität auf einzelne Lichtpunkte getestet und die mittlere Abweichung (= Mean Deviation $=$ MD) eines altersentsprechenden Normwertes angegeben. Als Normwert gilt eine generalisierte Absenkung der Empfindlichkeit von $+2 \mathrm{~dB}$ bis $\mathrm{zu}-2 \mathrm{~dB}$. Niedrigere Werte können auf einen Gesichtsfeldausfall hindeuten. Weiterhin wird die „Pattern standard deviation“ (= PSD) angegeben, welche die Homogenität des Gesichtsfeldes beschreibt. Die Normwerte liegen hier zwischen 1,8 $\pm 0,4 \mathrm{~dB}$. Die Untersuchung des Gesichtsfeldes ist stark abhängig von der bestmöglichen Visuseinstellung. Eine eventuell vorgesetzte Linse zur Korrektur einer Fehlsichtigkeit muss exakt positioniert sein, damit das Auge auch in der Peripherie noch in allen Bereichen durch die Linse blickt.

\subsubsection{Pachymetrie der Hornhaut}

Die Messung der Hornhautdicke, die Pachymetrie, erfolgt mit einem Ultraschallsensor, der auf die ebenfalls betäubte Hornhaut gesetzt wird. Der Schallkopf sendet, eine hochfrequente Impulswelle aus, die an der Hornhautvorder- und -rückfläche reflektiert wird. Der hoch empfindliche Sensor misst die zeitliche Differenz der reflektierten Impulswelle. Hierdurch kann der Abstand zwischen Vorder- und Rückfläche, die Hornhautdicke, berechnet werden. 


\section{Datenerfassung, Methoden und Durchführung}

\subsubsection{Nervenfaserschichtdickenmessung mittels optischer Kohärenztomographie (OCT)}

Die optischen Kohärenztomographie liefert als eine nichtinvasive Methode detaillierte Darstellungen der Makula und der Sehnervenregion, bei dem Licht geringer Kohärenzlänge mit Hilfe eines Interferometers zur Entfernungsmessung reflektiver Materialien eingesetzt wird. Diese Untersuchung erfolgt bei allen Patienten in Mydriasis.

In dieser Studie wird das Stratus OCT in der Version 4.0 der Firma Carl Zeiss Meditec, Jena, verwendet. Das Programm „Fast Retinal Nerve Fiber Layer“ misst mit kreisförmigen Scans von 3,4 mm Durchmesser je dreimal pro Auge an 256 Punkten die retinale Nervenfaserschichtdicke (RNFL) im peripapillären Bereich und beurteilt diese durch den Vergleich mit einer normativen, alterskorrelierten Datenbank. Die Ausgabe der Daten erfolgt in Ampelfarben, wobei „grün“ 90\% der Normalbevölkerung in dem jeweiligen Alter darstellt, „gelb“ grenzwertig bedeutet und „rot“ bei einem Prozent der Normalbevölkerung auftritt und somit unterhalb der Normgrenze liegt (Abb. 1 im Anhang).

\subsubsection{Muster-Elektroretinogramm (= Pattern-ERG = P-ERG)}

Das Muster-Elektroretinogramm, findet in einem abgedunkelten Raum mit standardisierter Restausleuchtung mit einem Multiliner $^{\circledR}$ Vision Gerät der Firma Toennies statt. Zur Geräteeinheit zählt ein Monitor, auf dem sich von einem Schachbrettmuster die hellen und dunklen Flächen abwechseln. Das Muster alterniert zwei mal pro Sekunde, was einer Wiederholungsrate von $1 \mathrm{~Hz}$ entspricht. Weiterhin ist an den Monitor ein Computer angeschlossen. Der Patient oder Proband sitzt $70 \mathrm{~cm}$ von dem Monitor entfernt. Die Haut an Ohrläppchen und Schläfen wird mit einer Abrasionspaste gereinigt. Als Nullelektrode bekommt die Person zwei Clipelektroden an die Ohrläppchen und zwei weitere zur Referenzbestimmung an die Schläfen (Abb. 2).

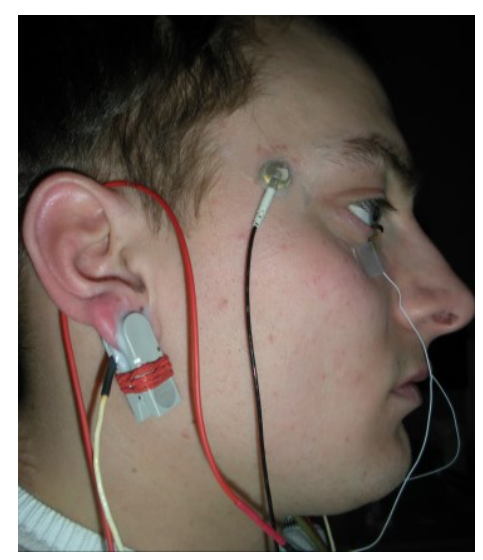

Abb. 2: Elektrodenanordnung rechts am Studienteilnehmer 


\section{Datenerfassung, Methoden und Durchführung}

Mit Ophtocain ${ }^{\circledR}-\mathrm{N}$-Augentropfen (Wirkstoff: Tetracain-HCl) wird die Hornhaut betäubt, damit je eine Goldfolienelektrode in den linken und rechten Bindehautsack gelegt werden kann (Abb. 3). Um einen direkten Kontakt der Goldfolienelektroden an der Hornhaut zu gewährleisten, dürfen bei dieser Untersuchung keine Kontaktlinsen getragen werden.

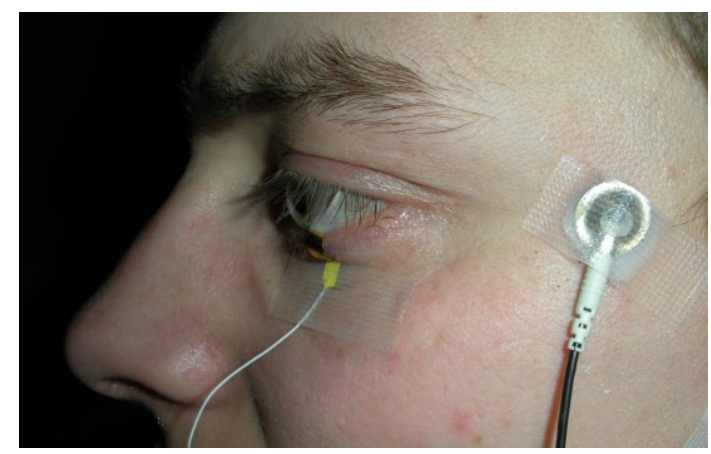

Abb. 3: Goldfolienelektrode im linken Bindehautsack und Referenzelektrode an der linken Schläfe

Es erfolgt eine automatische Impedanzmessung durch das Computerprogramm. Falls diese Messung mehr als $10 \mathrm{k} \Omega$ ergibt, müssen die Haut- oder Korneaelektroden überprüft und gegebenenfalls neu angebracht werden. Sobald der Grenzwert unterschritten wird, kann die Untersuchung gestartet werden.

Zur Messung fixiert die Person für circa 60 Sekunden einen Fixierpunkt in der Mitte des Monitors, wobei sich im Hintergrund die schwarzen und weißen Bereiche eines Schachbrettmusters umkehren. Da sich die beleuchteten Flächen während der Untersuchung nicht in ihrer Anzahl, sondern nur in ihrer Lokalisation vertauschen, bleibt die Gesamthelligkeit konstant. Damit ist die Reizantwort nicht durch die Helligkeit ausgelöst, sondern durch den Musterwechsel. Dieses Signal kann den Ganglienzellen zugeordnet werden, da durch die konstante Gesamthelligkeit On- und Off-Signale der Rezeptoren sich gegenseitig aufheben.

Der Kurvenverlauf eines normalen Muster-ERGs besteht aus einer kleinen negativen Welle nach etwa 35 ms (N35), gefolgt von einem deutlich größeren positiven Ausschlag nach etwa 40 bis 65 ms (P50), der nach etwa 95 ms in eine negative Komponente (N95) wechselt.

Zur Auswertung der Muster-ERGs wird die N95-Amplitude von der Spitze der P50Komponente zum Tal des N95-Auschlages gemessen. Hierbei beinhaltet die N95-Amplitude die Amplituden der P50- und der N35-Welle. Gemäß der International Society for Clinical 


\section{Datenerfassung, Methoden und Durchführung}

Electrophysiology of Vision (ISCEV) ist diese Messung ebenso üblich wie jene von einer selbstgewählten Nulllinie aus [26].

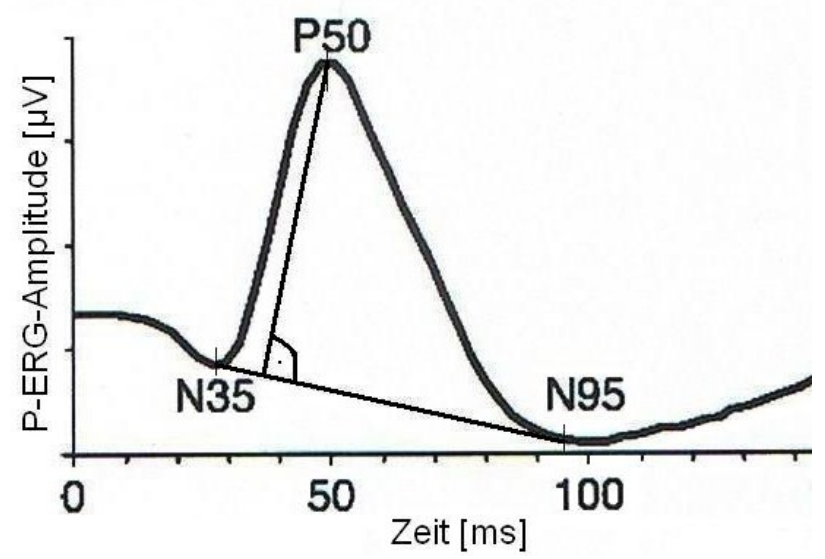

Abb. 4: Normales Muster-ERG mit Darstellung der N95-Amplitudenmessung [modifiziert aus 26]

Das Tal der N35-Komponente wird mit jenem der N95-Welle manuell verbunden und das Lot zum P50-Ausschlag gemessen. Die Angabe in mm wird gemäß der Skalierung in $\mu \mathrm{V}$ umgerechnet und ergibt die N95-Amplitude.

Die gemessenen Reizantworten sind sehr klein und anfällig gegenüber Störungen, wie z. B. Augenbewegungen, und werden daher über 128 Messungen durch ein komplexes computergestütztes Mittelungsverfahren berechnet. Anschließend kann der Kurvenverlauf durch Verrechnungen des Computerprogramms geglättet werden.

\subsubsection{Blaulicht-Muster-Elektroretinogramm (= Blaulicht-P-ERG)}

Das Blaulicht-Muster-Elektroretinogramm kombiniert eine Hypothese der Pathologie des Glaukoms mit einem Routineverfahren der Glaukomdiagnostik:

1) Eine glaukomassoziierte Schädigung der Nervenzellen macht sich frühzeitig in der Sensibilität der Blauzapfen bemerkbar [34]

2) Das Muster-ERG ist ein sensitiver Indikator für Nervenzellschäden [25].

Das Blaulicht-Muster-ERG wird ebenso wie das (Weiß-) Muster-ERG standardisiert gemäß den ISCEV-Richtlinien [26] durchgeführt und lediglich durch einen Blaulichtfilter ergänzt (Abb. 1). Dies ist ein blaues Glas, welches in dieser Studie aus einem Tischkoordinator nach Cüppers, einem orthoptischem Gerät zur Prüfung foveolarer Fixation, stammt. 


\section{Datenerfassung, Methoden und Durchführung}

Normalsichtigen Studienteilnehmern wird ein Brillengestell mit eingeschobenem blauen Glas auf der jeweiligen Seite aufgesetzt und Brillenträgern wird das gefärbte Glas direkt auf die Brille geklebt. Die Untersuchung erfolgt monokular, wobei das zweite Auge jeweils mit einer Papiervorlage abgedunkelt wird.

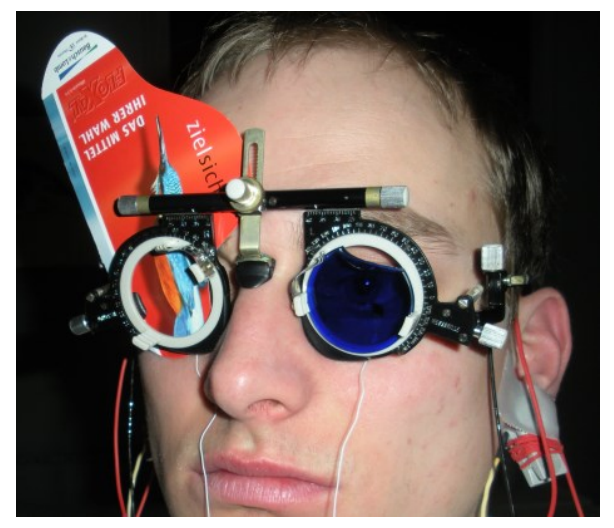

Abb. 5: Proband zur Messung des Blaulicht-Muster-ERGs

Das blaue Glas wirkt als optisches Filter. Es ist selektiv-transparent für Licht der Wellenlänge von etwa 350 bis $450 \mathrm{~nm}$. Es trifft daher annähernd monochromatisches violettes bis blaues Licht auf das Auge.

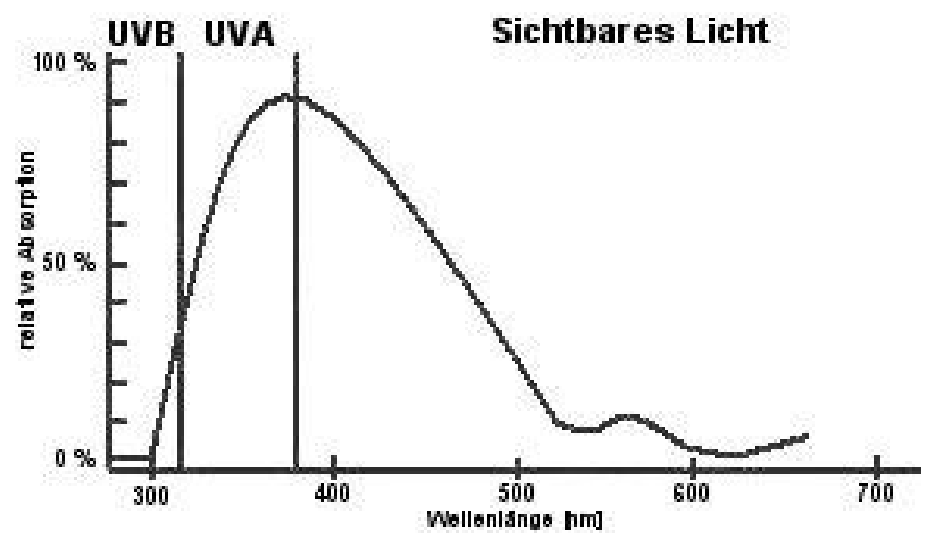

Abb. 6: Transmissiononsspektrum unseres verwendeten optischen Filters

Die mittels Spektrometer gemessene Wellenlänge unseres blaugefärbten Glases, welches zur Erzeugung monochromatischen Lichts genutzt wird.

Durch das verwendete Blaufilter erreicht man, dass nahezu selektiv Zapfen erregt werden, die den kurzwelligen Blaubereich des sichtbaren Farbspektrums absorbieren. Ein glaukombedingter selektiver Schaden des Blausinnes kann somit frühzeitig detektiert werden. 


\section{Datenerfassung, Methoden und Durchführung}

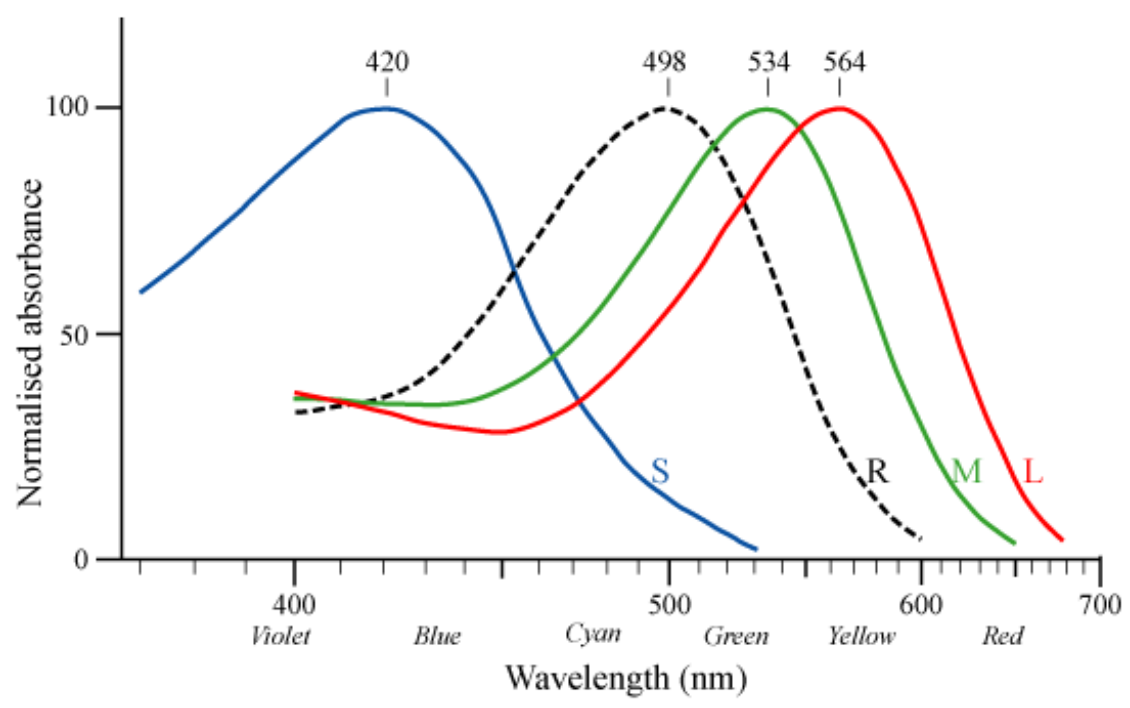

Abb. 7: Absorptionsspektrum der drei Farbrezeptoren und der Zapfen [35]

Spektrale Empfindlichkeit der Stäbchen (R) und Zapfen im kurzwelligen (S) Blau-, im mittelwelligen (M) Grünund im langwelligen (L) Rotbereich des Lichtes, jeweils relativ auf die maximale Empfindlichkeit der Sehzelle bezogen.

Solch ein im Blaulicht-P-ERG erkennbarer selektiver Blauzapfenschaden würde ebenfalls bei einer Farbfehlsichtigkeit für den Blausinn auffallen. Eine Blauschwäche (Tritanomalie) oder Blaublindheit (Tritanopie) ist sehr selten und kommt bei 0,002\% der Männer und 0,001\% der Frauen vor [36]. Demnach sind in der deutschen Bevölkerung nur etwa 1.600 Menschen betroffen. Während dieser Studie wird bei den Teilnehmern eine Blauschwäche oder -blindheit dadurch ausgeschlossen, dass sie die blauspezifischen Velhagen-Farbtafeln vorlesen müssen. Diese Tafeln wurden von allen Studienteilnehmern korrekt erkannt.

\subsection{Auswertungsverfahren}

Zur Auswertung werden die Ergebnisse der durchschnittlichen Nervenfaserschichtdicke im OCT der Amplitudenveränderung des Blaulicht-Muster-ERGs als dichotome Merkmale „gesund“ oder „erkrankt/pathologisch“ gegenübergestellt. Im OCT gilt jemand als ,erkrankt“, wenn die RNFL kleiner als 5\% (= gelb), bzw. kleiner als 1\% (= rot) bezugnehmend auf die Normalbevölkerung klassifiziert wird (Abb. 8). Dies bezieht sich sowohl auf die Auswertung der Einzelsegmente (Kreisdiagramme in Abb. 8) als auch auf den Mittelwert der Gesamtnervenfaserschichtdicke (Angabe der „Avg. Thickness” in Abb. 8).

Beispielsweise ist folglich eine durchschnittliche Nervenfaserschichtdicke von 76,54 $\mu \mathrm{m}$ wie auch jene von $80,65 \mu \mathrm{m}$ bei einem 18-jährigen Studienteilnehmer im alterskorrelierten Vergleich als ,pathologisch“ zu bewerten (Abb. 8; ID 202). 


\section{Datenerfassung, Methoden und Durchführung}

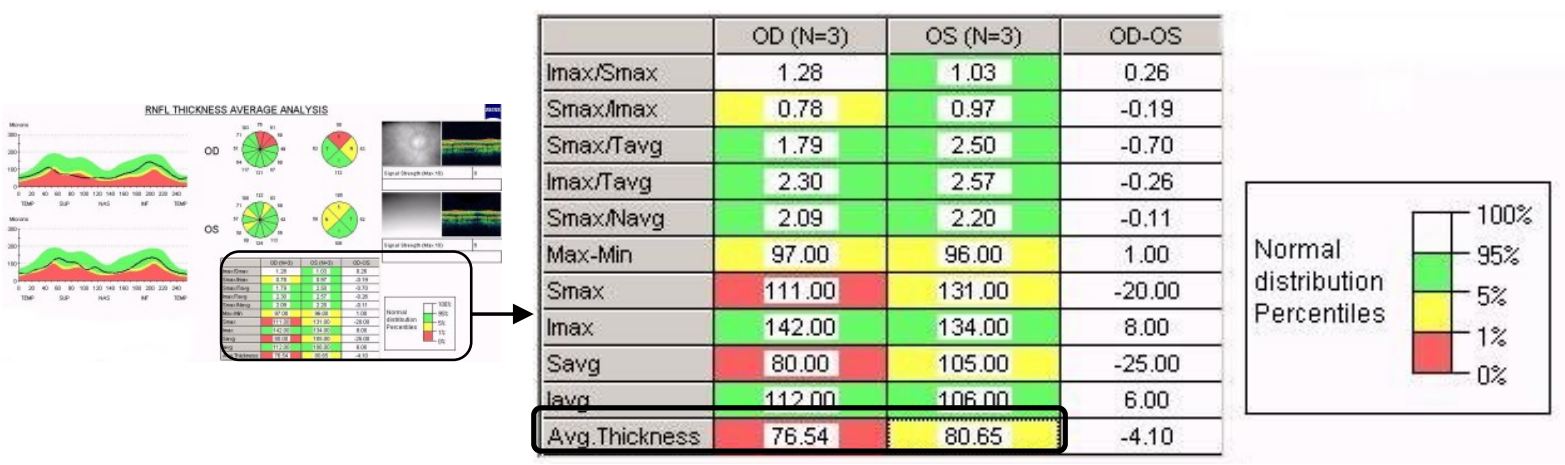

\section{Abb. 8: Berechnung der durchschnittlichen Nervenfaserschichtdicke mittels Stratus OCT}

Die altersbezogene Farbkodierung der durchschnittlichen Nervenfaserschichtdicke (Avg. Thickness) zeigt an, dass die durchschnittliche Nervenfaserschichtdicke dieses Patienten als ,pathologisch" zu werten ist ( $\mathrm{OD}=$ rot $=$ unterhalb der $1 \%$ Perzentile, OS $=$ gelb $=$ unterhalb der 5\% Perzentile) (ID 202).

Beim Blaulicht-Muster-ERG erfolgt zunächst die Bestimmung der Relation zum MusterERG. Hierbei wird die Reduktion der Amplitude durch den Blaulichtfilter berechnet (Abb. 9 und 10). Dieses Auswertungsverfahren ist somit interindividuell vergleichbar.

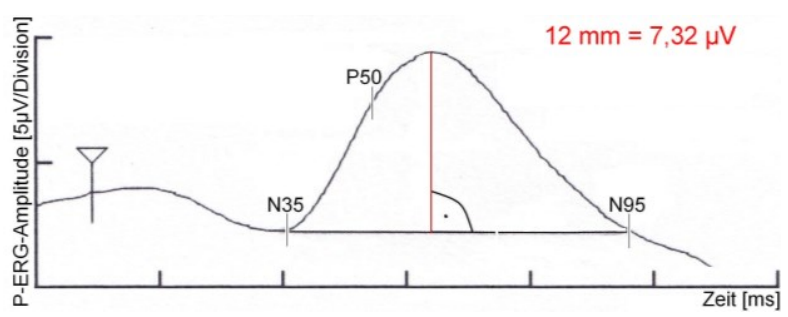

Abb. 9: N95-Amplitude eines Weiß-Muster-ERGs bei einem gesunden Probanden

Der Abstand von einem Teilstrich zum folgenden der y-Achse beträgt im Original 8,2 mm und entspricht $5 \mu \mathrm{V}$. Demnach ergeben $12 \mathrm{~mm}$ der N95-Amplitude ein Weiß-P-ERG-Potential von 7,32 $\mu \mathrm{V}$ (ID 15).

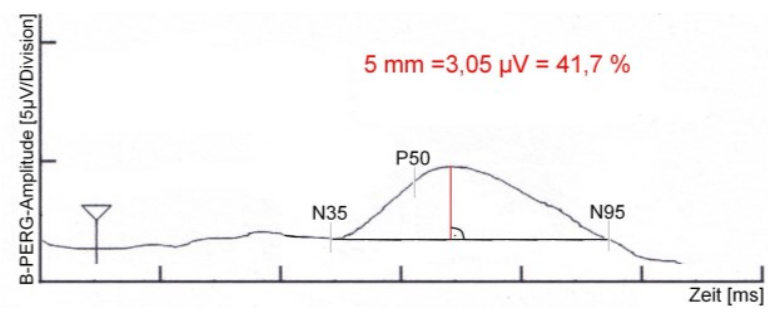

Abb. 10: N95-Amplitude eines Blaulicht-Muster-ERGs bei einem gesunden Probanden

Die N95-Amplitude des Blaulicht-P-ERGs $(3,05 \mu \mathrm{V})$ ist gegenüber dem Weiß-P-ERG auf 41,7\% reduziert (ID 15).

Da wir eine Abnahme der Amplitude des Blaulicht-Muster-ERGs durch die senile Katarakt erwarten, analysieren wir die Ergebnisse des Blaulicht-Muster-ERGs von 30 


\section{Datenerfassung, Methoden und Durchführung}

Normalprobanden mittels $\mathrm{R}$ [37] und berechnen eine Regressionsgerade, um die physiologische Abnahme des Blaulicht-Muster-ERGs durch Linsentrübung in Abhängigkeit vom Alter darzustellen. Weiterhin werden Ober- und Untergrenzen eines 90\%Referenzbereiches berechnet, in dem sich etwa 90\% der gesunden Normalbevölkerung befinden. Daraus ergeben sich für jedes Alter geschätzte Normbereiche, anhand derer die Entscheidung „gesund“ oder „pathologisch“ laut Blaulicht-Muster-ERG getroffen werden kann.

Um das Blaulicht-Muster-ERG mit der optischen Kohärenztomographie zu vergleichen, wurden die Ergebnisse beider Tests in einer Kreuztabelle einander gegenübergestellt. Als Maß für die Übereinstimmung der Verfahren wurde Cohens Kappa-Koeffizient [38] mittels SPSS für Windows, Version 16.0, berechnet. 


\section{Ergebnisse}

\subsection{Muster-Elektroretinogramm}

\subsubsection{Gesunde Probanden}

Die Muster-ERG-Amplituden der 60 Augen von den 30 gesunden Studienteilnehmern wiesen eine große Spannweite (SW 1,8-9,8 $\mu \mathrm{V}, \mathrm{MW} 5,3 \mu \mathrm{V}, \mathrm{SD} \pm 1,8 \mu \mathrm{V}$ ) auf. Die Kurven des Blaulicht-Muster-ERGs zeigten deutlich geringere Amplituden (SW 0-5,8 $\mu \mathrm{V}$, MW 2,7 $\mu \mathrm{V}$, $\mathrm{SD} \pm 1,4 \mu \mathrm{V})($ Abb. 11, Tabelle 6 im Anhang).

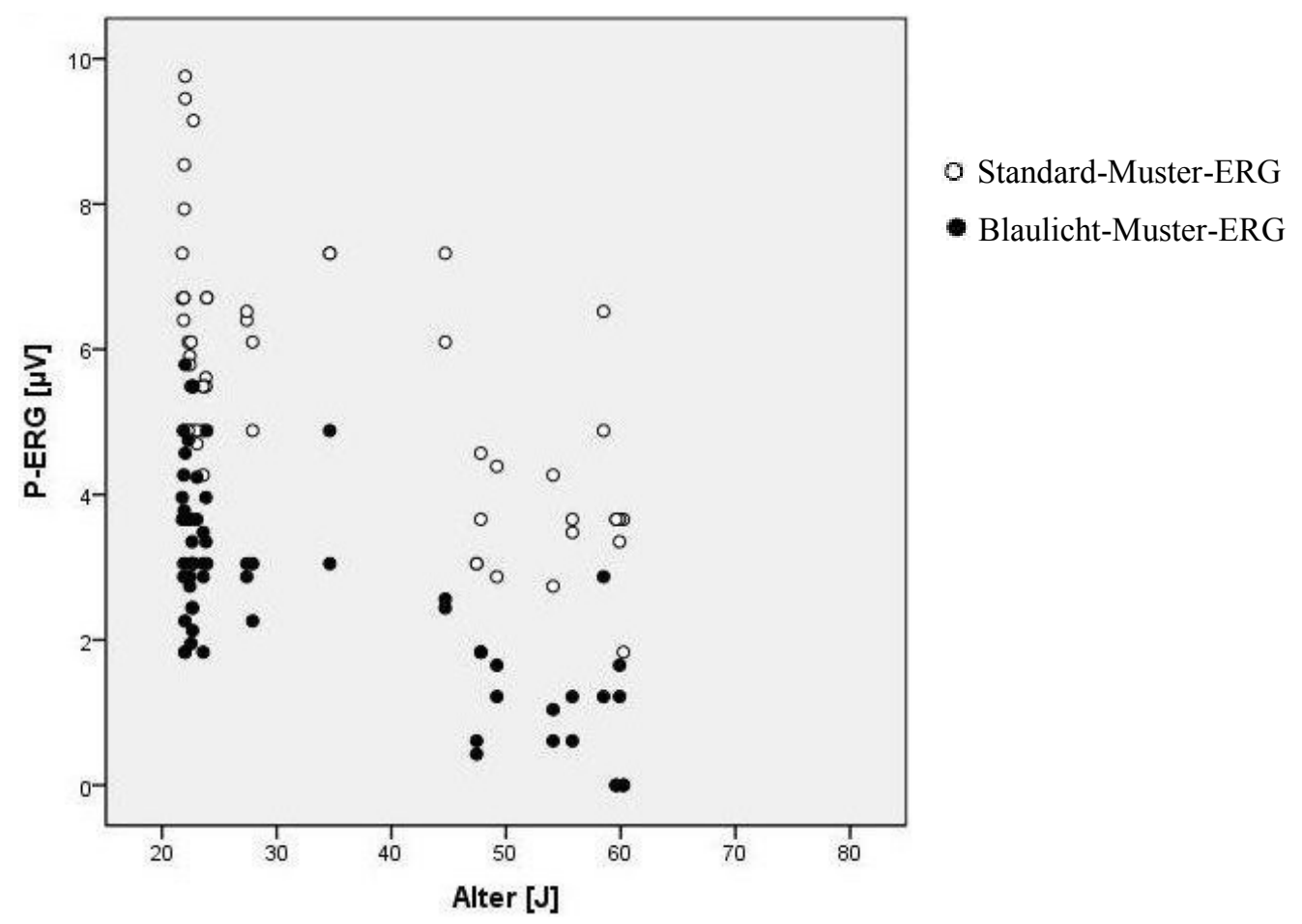

\section{Abb. 11: P-ERG-Messungen in Abhängigkeit vom Alter bei Normalprobanden}

Die Amplitude des Standard-(weiß-)Muster-ERGs (०) ist in allen Altersklassen messbar, wohingegen die des Blaulicht-Muster-Elektroretinogramms $(\bullet)$ tendenziell mit zunehmendem Alter sinkt bis sie ganz erlischt.

\subsubsection{Alterskorrelierte Verteilung des Blaulicht-Muster-ERGs}

Die prozentuale Reduktion des Blaulicht-P-ERGs verglichen mit dem Standard-P-ERG, das Blau- zu Weiß-Muster-ERG (=BW-M-ERG), nimmt mit steigendem Alter ab. Dieser näherungsweise negativ lineare Zusammenhang wird durch eine Regressionsgerade veranschaulicht (Abb. 12) und erlaubt die statistische Berechnung einer alterskorrigierten Verteilungskurve. 


\section{Ergebnisse}

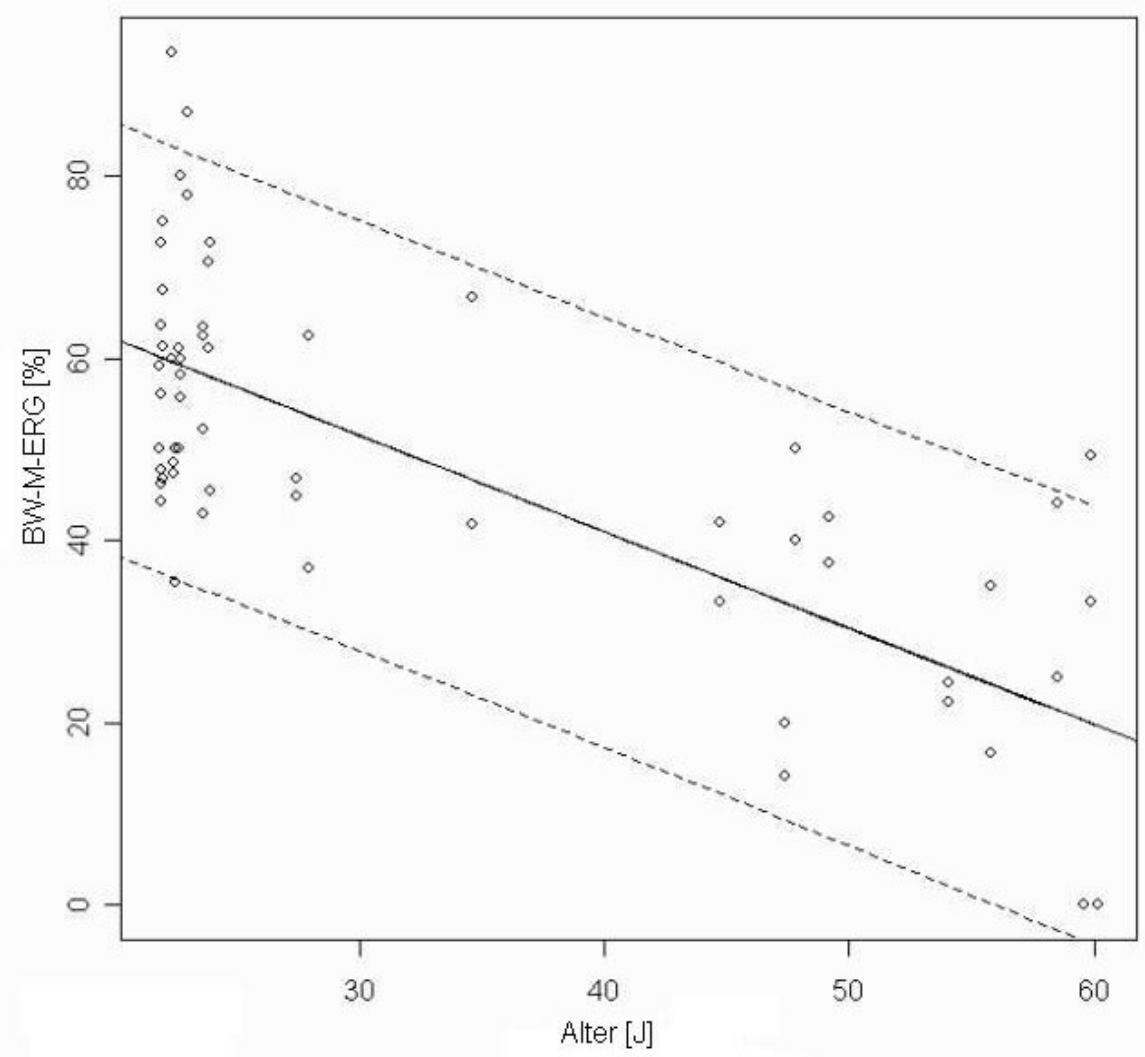

Abb. 12: Alterskorrigierte Eichkurve für die Katarakt erstellt an Normalprobanden

Das Potential des Blau-zu-Weiß-Muster-Elektroretinogramms (BW-M-ERG) sinkt stetig mit zunehmendem Alter durch die physiologische Trübung der Linse. Mittels Regressionsgerade und 90\%-Referenzbereich ergeben sich Grenzwerte für die Beurteilung des Blaulicht-Muster-ERGs.

Unter Beachtung der Regressionsanalyse kann für jedes Alter ein konkreter Blau-zu-WeißMuster-ERG-Normalwert geschätzt werden. Aufgrund der großen interindividuellen Schwankungen wurde ein 90\%-Referenzbereich gewählt, in dem somit etwa $90 \%$ der gesunden Normalbevölkerung liegen. Unter Zuhilfenahme der Programmiersprache R [37] wurden für jedes Alter zwischen 20 und 60 Jahren die Ober- und Untergrenzen eines so definierten physiologischen BW-M-ERG-Wertes unter Annahme eines linearen Modells geschätzt (Tab. 7). Da ab einem Alter von 56 Jahren die physiologische Trübung der Linse teilweise soviel blaues Licht absorbiert, dass das Blaulicht-P-ERG auch bei sicher gesunden Studienteilnehmern erniedrigt ist, legten wir die Grenze zur Auswertung der BlaulichtMuster-ERGs auf maximal 56 Jahre fest. Somit schieden vier gesunde Probanden aufgrund ihres Alters aus. Die verbleibenden 26 Normalprobanden wurden alle als ,gesund“ laut BWM-ERG klassifiziert. 


\section{Ergebnisse}

\subsubsection{Kataraktpatienten}

Nachdem der Einfluss der physiologischen Linsentrübung auf die ERG-Untersuchung deutlich wurde, untersuchten wir in einem Nebenarm der Studie zwei gesunde wie auch zwei Glaukompatienten vor beziehungsweise nach ihrer Kataraktoperation (Tabelle 8 im Anhang).

\subsubsection{Gesunde Kataraktpatienten}

Eine gesunde 53-jährige Patientin (auf dem rechten Auge pseudophak, links vor Kataraktoperation) zeigt bei der ersten Untersuchung rechts einen deutlichen Kurvenverlauf mit einer Amplitudenreduktion von initial 2,7 $\mu \mathrm{V}$ im Standard-P-ERG auf 2,3 $\mu \mathrm{V}$ im Blaulicht-P-ERG und links ein erloschenes ERG sowohl im Standard- wie auch im BlaulichtP-ERG. Bei der zweiten Untersuchung 4 Monate nach der Kataraktoperation des linken Auges zeigt sich auch im Blaulicht-P-ERG ein klarer Kurvenverlauf. Die Amplitude von 3,1 $\mu \mathrm{V}$ des Standard-P-ERGs sinkt auf 2,1 $\mu \mathrm{V}$ im Blaulicht-PERG.

Im Alter von 60 Jahren erreicht die zweite gesunde Kataraktpatientin postoperativ im Standard-P-ERG rechts 5,2 $\mu \mathrm{V}$, links $6,7 \mu \mathrm{V}$ sowie im Blaulicht-P-ERG rechts $1,2 \mu \mathrm{V}$, links $2,4 \mu \mathrm{V}$.

\subsubsection{Glaukomerkrankte Kataraktpatienten}

Zwei einseitig pseudophake Glaukompatienten zeigen im Standard-MusterElektroretinogramm erniedrigte bis normale Kurven bis $4,9 \mu \mathrm{V}$, jedoch im Blaulicht-MusterERG in beiden Fällen nicht mehr messbare Potentiale.

\subsubsection{Glaukompatienten}

In der Gruppe der 20 Glaukompatienten ist das Blaulicht-Muster-Elektroretinogramm (SW 0$2,1 \mu \mathrm{V}, \mathrm{MW} 0,3 \mu \mathrm{V}, \mathrm{SD} \pm 0,6 \mu \mathrm{V})$ meist vollständig erloschen und das Standard-MusterERG (SW 0-5,5 $\mu \mathrm{V}, \mathrm{MW} 2,5 \mu \mathrm{V}, \mathrm{SD} \pm 1,5 \mu \mathrm{V}$ ) deutlich kleiner als in der Gruppe der gesunden Studienteilnehmer (Abb. 13, Tabelle 9 im Anhang). 


\section{Ergebnisse}

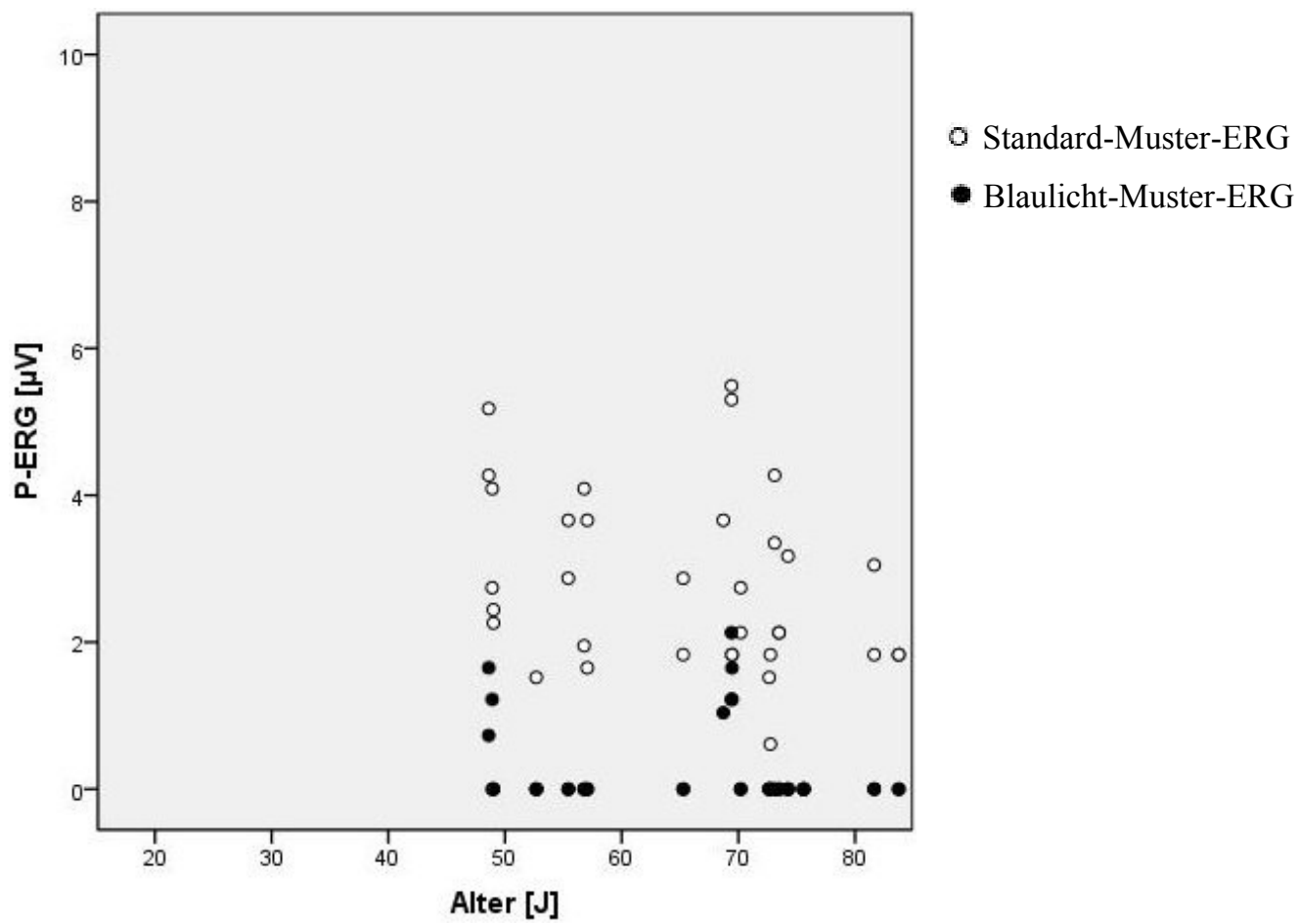

Abb. 13: P-ERG-Messungen in Abhängigkeit vom Alter bei Glaukompatienten

Das Standard-Muster-ERG (०) ergibt Amplituden im gut messbaren Bereich, während das Blaulicht-MusterERG $(\bullet)$ sehr geringe bis gar keine Ausschläge zeigt.

\subsubsection{Studienteilnehmer mit Glaukomverdacht}

Die Ergebnisse der Muster-ERG-Messungen bei den 20 Glaukomverdachtspatienten zeigten ein sehr heterogenes Bild, sodass eine Unterteilung in „eher glaukommanifeste“ und „eher gesunde" Glaukomverdachtspatienten erfolgte (Abb. 14 und 15, Tabelle 10 im Anhang). Das Muster-ERG ergab durchschnittlich Amplituden von 4,3 $\mu \mathrm{V}(\mathrm{SW} 0-10,4 \mu \mathrm{V}, \mathrm{SD} \pm 2,0 \mu \mathrm{V})$ und jene des Blaulicht-Muster-ERGs von 1,6 $\mu \mathrm{V}(\mathrm{SW} 0-3,7 \mu \mathrm{V}, \mathrm{SD} \pm 1,2 \mu \mathrm{V})$. 


\section{Ergebnisse}

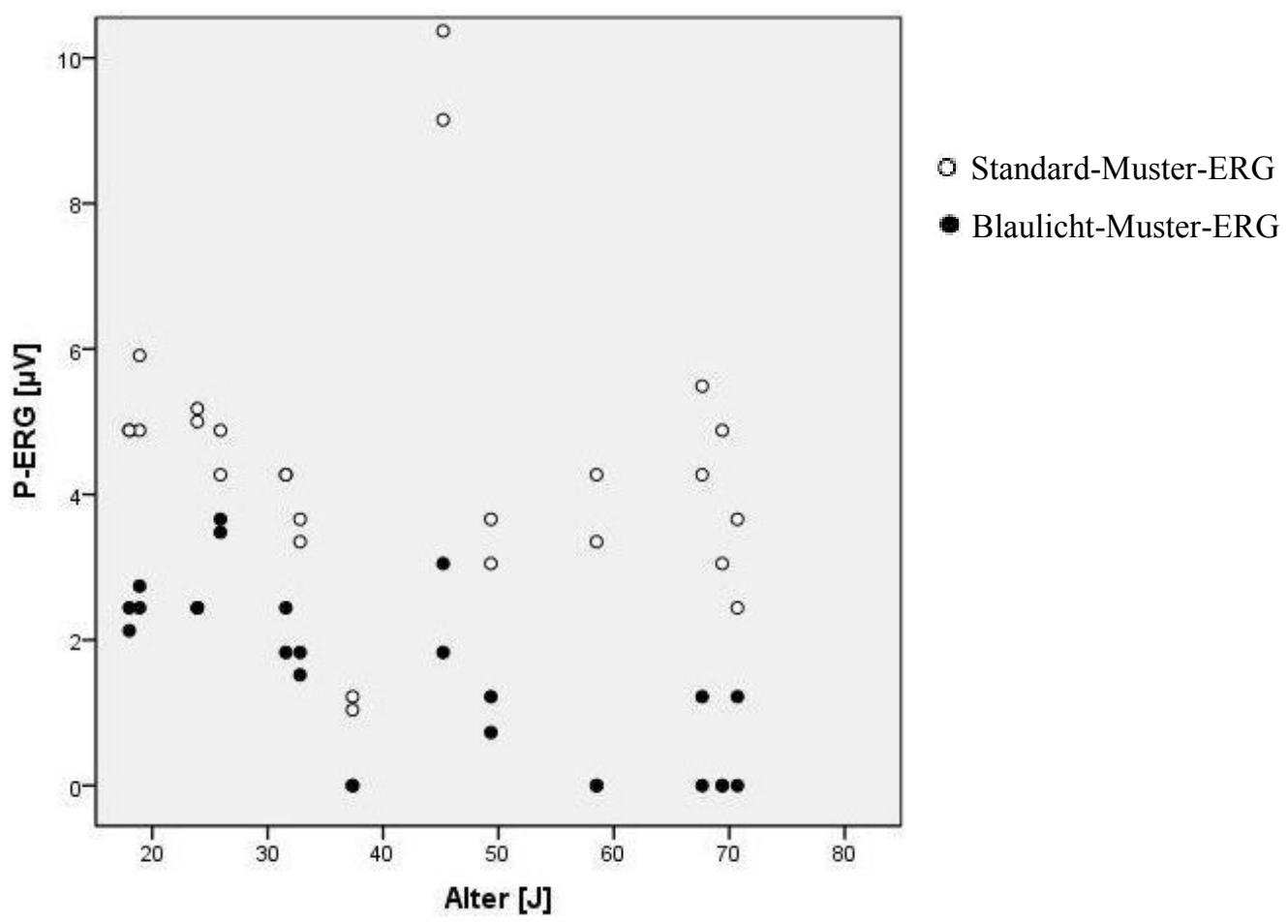

Abb. 14: P-ERG-Messungen in Abhängigkeit vom Alter bei „eher glaukommanifesten“ Glaukomverdachtspatienten

Es ergibt sich eine heterogene Verteilung der Amplituden des Standard-Muster-ERGs (O) und des BlaulichtMuster-ERGs $(\bullet)$, wobei sich eine Tendenz zu eher geringeren Blaulicht-Muster-ERG-Amplituden abzuzeichnen scheint.

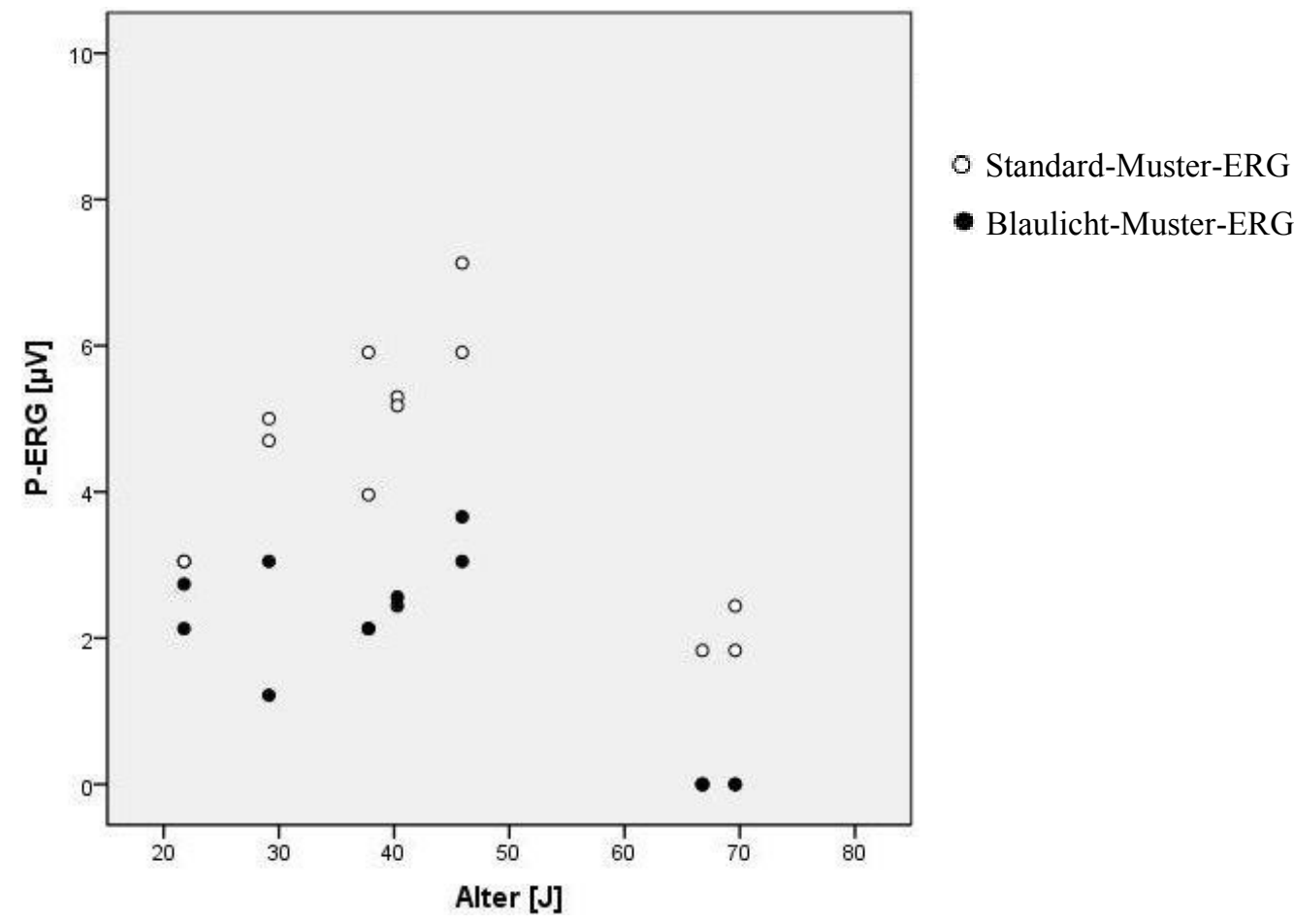

Abb. 15: P-ERG-Messungen in Abhängigkeit vom Alter bei „eher gesunden“ Glaukomverdachtspatienten

Die Amplituden des Standard-Muster-ERGs (०) und des Blaulicht-Muster-ERGs (•) liegen graphisch zwischen dem Bild eines Normalprobanden (Abb. 11) und dem eines Glaukompatienten (Abb. 13), wobei die P-ERGAmplituden hier eher zu ,gesunden Werten“ tendieren. 


\section{Ergebnisse}

\subsection{Nervenfaserschichtdickenmessung mittels Stratus OCT}

\subsubsection{Gesunde Probanden}

Für die OCT-Messung werden fünf der 30 gesunden Normalprobanden ausgeschlossen, drei aufgrund eines schrägen Sehnerveneintrittes und zwei weitere absolvierten nicht alle notwendigen Untersuchungen. Die Sehnervenfaserschichtdicke wird folglich in 50 Augen von 25 gesunden Studienteilnehmern gemessen und beträgt durchschnittlich 99,06 $\mu \mathrm{m}$ ( $\mathrm{SD} \pm$ 9,68 $\mu \mathrm{m})$. Die RNFL wurde bei jedem Probanden mit „grün“ kodiert und lag somit im Normbereich verglichen mit der altersbezogenen Datenbank des systemeigenen Programms. Infolgedessen wurden alle Normalprobanden bezugnehmend auf die retinale Nervenfaserschichtdicke als ,gesund“ identifiziert (Tabelle 11, Tabelle 6 im Anhang).

Tabelle 11: RNFL-Ergebnisse der Normalprobanden

\begin{tabular}{|c|c|c|c|c|c|}
\cline { 2 - 6 } \multicolumn{1}{l|}{} & $\mathrm{n}=$ & Minimum & Maximum & Mittelwert & Standardabweichung \\
\hline RNFL & 50 & $82,58 \mu \mathrm{m}$ & $120,97 \mu \mathrm{m}$ & $99,06 \mu \mathrm{m}$ & $9,68 \mu \mathrm{m}$ \\
\hline
\end{tabular}

\subsubsection{Glaukompatienten}

Von den 20 Teilnehmern der Gruppe III ist eine Patientin bereits an einem Auge pseudophak, sodass in die Auswertung dieser Ergebnisse nur das nicht operierte glaukomerkrankte Auge einbezogen wird. Von den somit relevanten 39 Augen ist die RNFL 18mal als „grenzwertig“ (= gelb) oder ,pathologisch“ (= rot) klassifiziert worden (Tabelle 12, Tabelle 9 im Anhang). Mit 76,15 $\mu \mathrm{m}(\mathrm{SD} \pm 14,74 \mu \mathrm{m})$ ist die durchschnittliche Nervenfaserschichtdicke dieser Gruppe sichtlich geringer als jene des Normalkollektivs mit annähernd $100 \mu \mathrm{m}$.

Tabelle 12: RNFL-Ergebnisse der Glaukompatienten

\begin{tabular}{|c|c|c|c|c|c|c|c|}
\cline { 2 - 7 } \multicolumn{1}{c|}{} & $\mathrm{n}=$ & Minimum & Maximum & Mittelwert & Standardabweichung & Gesund & Pathologisch \\
\hline RNFL & 39 & $44,24 \mu \mathrm{m}$ & $110,37 \mu \mathrm{m}$ & $76,15 \mu \mathrm{m}$ & $14,74 \mu \mathrm{m}$ & $21(53,8 \%)$ & $18(46,2 \%)$ \\
\hline
\end{tabular}

\subsubsection{Studienteilnehmer mit Glaukomverdacht}

Bei den 40 Augen der 20 Glaukomverdachtspatienten ergibt auch die Nervenfaserschichtdicken-Messung sehr unterschiedliche Werte (MW 92,83, 


\section{Ergebnisse}

$\mathrm{SD} \pm 11,83 \mu \mathrm{m}) .31$ Augen (=77,5\%) wurden als ,gesund“ und 9 Augen als ,pathologisch“ bewertet (Tabelle 13).

Tabelle 13: RNFL-Ergebnisse der Glaukomverdachtspatienten

\begin{tabular}{|c|c|c|c|c|c|c|c|}
\cline { 2 - 7 } \multicolumn{1}{c|}{} & $\mathrm{n}=$ & Minimum & Maximum & Mittelwert & Standardabweichung & Gesund & Pathologisch \\
\hline RNFL & 40 & $56,69 \mu \mathrm{m}$ & $112,66 \mu \mathrm{m}$ & $92,83 \mu \mathrm{m}$ & $11,83 \mu \mathrm{m}$ & $31(77,5 \%)$ & $9(22,5 \%)$ \\
\hline
\end{tabular}

\section{3 Übereinstimmung von OCT und BW-M-ERG}

Von den anfangs 70 Studienteilnehmern (140 Augen) konnten schließlich noch 82 Augen eingeschlossen werden. Sechs Augen entfielen bei schrägem Sehnerveneintritt, vier, da kein OCT durchführbar war und 48 weitere Augen wurden später aus der Wertung genommen, da die Teilnehmer älter als 56 Jahre waren und ab dann eine Differenzierung zwischen glaukomtypisch und durch die Linsentrübung bedingt erloschenem BW-M-ERG nicht möglich ist.

Knapp zwei Drittel der 82 untersuchten Fälle sind gesund, während das andere Drittel aus Glaukompatienten und aus Patienten, bei denen ein Glaukom eher manifest zu sein scheint, besteht (Tabelle 14).

Tabelle 14: Häufigkeitsverteilung der Studienteilnehmer in der Endauswertung

\begin{tabular}{|l|c|c|}
\cline { 2 - 3 } \multicolumn{1}{c|}{} & $\mathrm{n}=$ & $\%$ \\
\hline I) Augen gesunder Probanden & 44 & 53,7 \\
\hline II) Augen von Glaukompatienten & 10 & 12,2 \\
\hline III) Augen von Verdachtspatienten & & \\
a) "eher Glaukom manifest" & 18 & 22,0 \\
b) "eher kein Glaukom" & 10 & 12,2 \\
\hline Gesamt & 82 & 100,0 \\
\hline
\end{tabular}

Zum Vergleich des neuen Verfahrens des Blaulicht-Muster-ERGs mit der bekannten Methode der Nervenfaserschichtdickenmessung mittels OCT wurden die Ergebnisse (,gesund“ oder „pathologisch“) beider Verfahren in einer Kreuztabelle gegenübergestellt (Tabelle 15). 


\section{Ergebnisse}

Tabelle 15: Kreuztabelle der diagnostischen Urteile mittels retinaler Nervenfaserschichtdickenmessung und Blau-zu Weiß-Muster-ERG

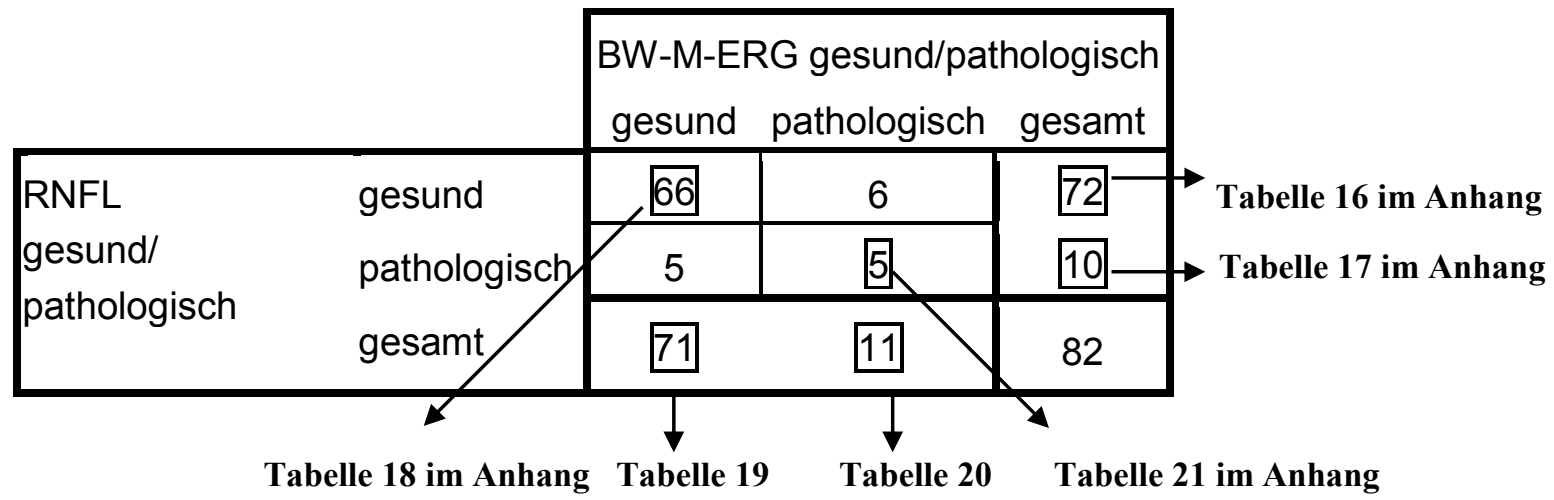

Weiterhin erfolgt die Zuordnung der Enddiagnosen der beiden Verfahren zu der zum Zeitpunkt der Untersuchung geltenden Gruppenzugehörigkeit (,gesund“, „pathologisch“, „eher Glaukom manifest“, „eher kein Glaukom“) (Tabelle 22).

Tabelle 22: ERG- und RNFL-Ergebnisse im Vergleich zum klinischen Befund

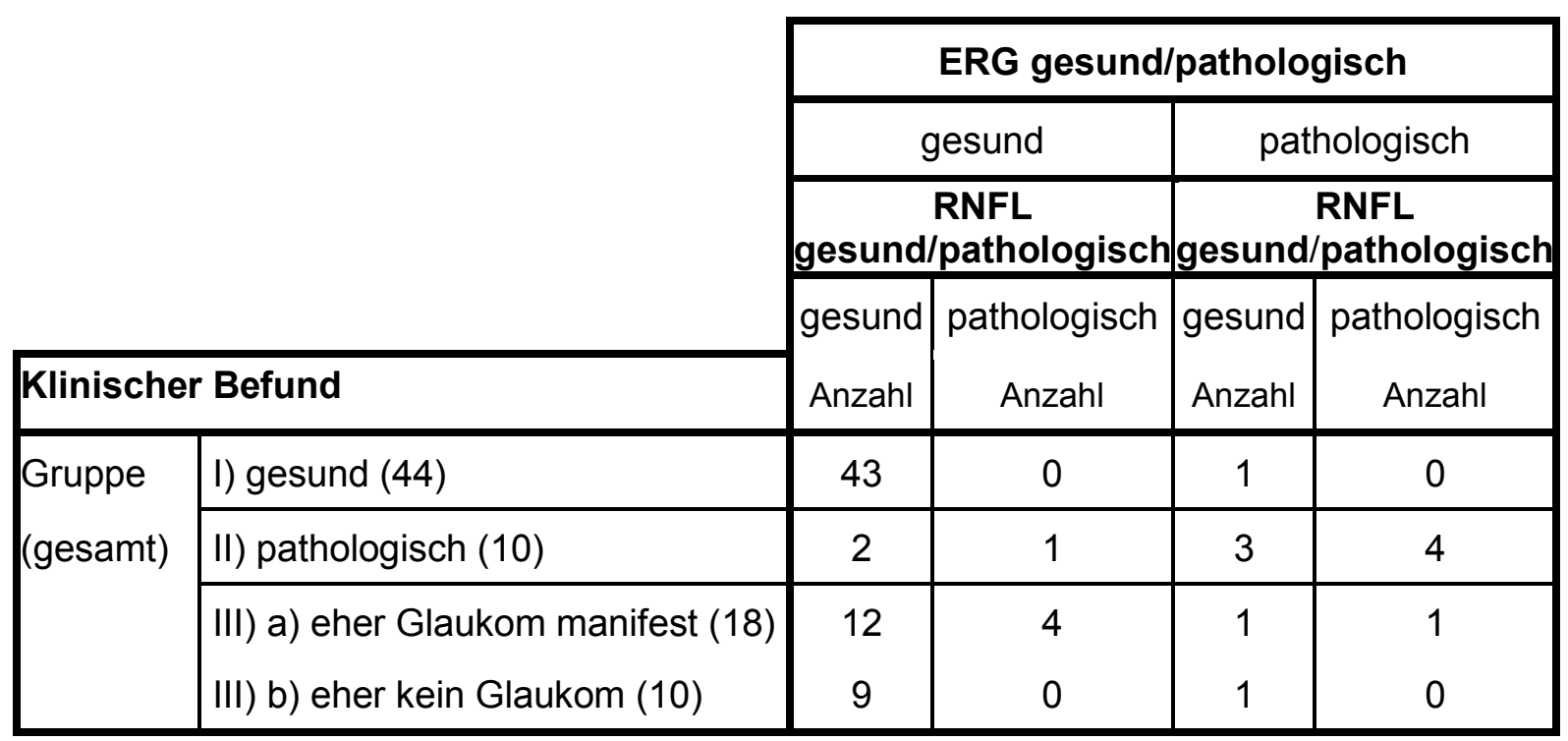

Dadurch lassen sich Sensitivität und Spezifität der Verfahren schätzen. Das ERG-Verfahren erkannte 7 von 10 richtig als ,pathologisch“, demnach ergab sich eine geschätzte Sensitivität von $70 \%$. Die RNFL-Messung bewertete hingegen nur 5 von den 10 als ,pathologisch“, sodass hier eine Sensitivität von 50\% geschätzt werden konnte. Das ERG klassifizierte 43 von 44 Augen als ,gesund“ und hatte somit eine geschätzte Spezifität von 97,7\%. Die OCTUntersuchung bezeichnete alle 44 gesunden Augen korrekt, folglich errechnete sich hier eine geschätzte Spezifität von 100\%. 


\section{Ergebnisse}

Um das Blaulicht-Muster-ERG der RNFL-Messung mittels Stratus OCT hinsichtlich ihrer endgültigen Aussagen abschließend gegenüber zu stellen, wird Cohens Kappa-Koeffizient $\kappa$ als Maß für die Übereinstimmung beider Verfahren bestimmt. Dieser Wert berücksichtigt die zufällig zu erwartende Kongruenz für die Bewertung der beobachteten Übereinstimmung. Für den Fall dichotomer Merkmale, wie in dieser Studie ,gesund“ und „pathologisch“, erfolgt entsprechend statistischer Auswertung eine Schätzung für das $\kappa$ aus den Werten der Tabelle 15 [39].

Tabelle 23: Cohens Kappa-Koeffizient $\kappa$

\begin{tabular}{|c|c|c|c|}
\hline & & Wert & $\begin{array}{l}\text { Asymptotischer } \\
\text { Standardfehler }\end{array}$ \\
\hline Maß der Übereinstimmung & $\begin{array}{l}\text { Kappa } \\
\text { Anzahl der gültigen Fälle }\end{array}$ & $\begin{array}{c}0,399 \\
82\end{array}$ & 0,148 \\
\hline
\end{tabular}

Mit Hilfe des angegebenen Standardfehlers von 0,148 wird das asymptotische 95\%-Konfidenzintervall für $\kappa$ bestimmt [39]:

Obergrenze:

$\kappa+1,96 \cdot 0,148=0,69$

Untergrenze:

$\kappa-1,96 \cdot 0,148=0,11$

0,11 und 0,69 bilden Unter- und Obergrenzen des 95\%-Konfidenzintervalls für das Übereinstimmungsmaß Cohens Kappa. 


\section{Diskussion}

\section{Diskussion}

\subsection{Das Muster-Elektroretinogramm heute in der medizinischen Anwendung}

Das Muster-ERG ist ein gängiges, in die Augenheilkunde eingeführtes Verfahren. Es wird sowohl in der Differentialdiagnostik von Optikopathien, beispielsweise beim Glaukom, als auch bei Makulopathien eingesetzt.

Bach et al. untersuchten 2006 das Muster-Elektroretinogramm als frühen Glaukomindikator bei Patienten mit okulären Hypertensionen [25]. Sie werteten über einen durchschnittlichen Zeitraum von 8,2 Jahren die Gesichtsfelder und das P-ERG von 95 Augen von 54 Patienten mit einem IOD $\geq 25 \mathrm{mmHg}$, normalem Gesichtsfeld, physiologisch exkavierter Papille und einer Sehschärfe $\geq 0,8$ aus. Bach und seine Mitarbeiter setzten die Ergebnisse von MusterElektroretinogrammen mit unterschiedlichen Schachbrettmustergrößen zueinander in Beziehung. Im Ergebnis entwickelten acht Augen (fünf Patienten) glaukomtypische Gesichtsfeldausfälle. Rückblickend berechneten sie für das P-ERG ein Jahr vor der Glaukomerkrankung eine Sensitivität von $80 \%$ und eine Spezifität von $71 \%$.

Weiterhin wird dieses elektrophysiologische Verfahren bei neurologischen Erkrankungen angewendet.

So schrieb die Arbeitsgruppe um Nowacka 2010 über Untersuchungen bei Parkinsonpatienten [40]. Diese entwickeln häufig visuelle Fehlwahrnehmungen sowohl bedingt durch eine SiccaSymptomatik der Hornhaut als auch durch ein Glaukom. Elektrophysiologische Analysen lassen hier die Differentialdiagnose eines Glaukoms feststellen.

Ebenso werteten Krasodomska et al. 2010 unter anderem das P-ERG bei Patienten mit Morbus Alzheimer aus [41]. Diese Patienten beklagen oft Sehverschlechterungen, die mit routinemäßigen ophthalmologischen Untersuchungen nicht erklärbar sind. Die häufigste Ursache sind neuropathologische Veränderung im visuellen Kortex. Dessen ungeachtet können Erkrankungen der Retina oder des Sehnerven nicht ausgeschlossen werden. Das Muster-ERG (und andere Untersuchungen wie das Muster-VEP) zeigte eine reduzierte N95Amplitude sowie eine gesteigerte P100-Latenz bei 17 von 30 Alzheimerpatienten, sodass Krasodomska et al. einen Ganglienzell- und Sehnervenschaden im frühen Stadium der Alzheimererkrankung vorhanden sahen.

Einen interessanten Ansatz fanden Bubl und Kollegen 2010 in ihrer Studie „Seeing gray when feeling blue? Depression can be measured in the eye of the diseased" [42]. An der 


\section{Diskussion}

Untersuchung nahmen 80 Personen teil, 40 davon mit Major Depression (20 Personen unter antidepressiver Medikation, 20 Patienten ohne) und 40 gesunde Teilnehmer. Mittels MusterElektroretinogramm wurde die Reaktion der Netzhaut auf sich ändernde Schwarz-WeißKontraste des Schachbrettes gemessen. Die Ergebnisse zeigten deutlich, dass alle depressiven Patienten (unabhängig von einer vorhandenen oder nicht vorhandenen Therapie) eine signifikant geringere Kontrastwahrnehmung hatten als gesunde Personen aus der Kontrollgruppe. So konnte mit objektiven Methoden nachgewiesen werden, dass Menschen mit einer Depression tatsächlich in einer grauen Welt leben.

\subsubsection{Muster-ERG bei gesunden Probanden}

In dieser Studie zeigen die Ergebnisse im Standard-Muster-ERG bei Normalprobanden im Mittel eine Amplitudenreduktion von initial 5,3 $\mu \mathrm{V}$ auf 2,6 $\mu \mathrm{V}$ im Blaulicht-Muster-ERG.

Dies bedeutet, dass sich allein durch die Verwendung eines Blaufilters die Potenziale des P-ERGs in gesunden Augen etwa halbieren. Dies entspricht über alle Altersklassen gemittelt einer physiologischen, reduzierten Antwort der Ganglienzellen auf 49\%.

Dies ist begründet durch das verwendete optische Blaufilter, welches nur für Licht der Wellenlänge 350 - $450 \mathrm{~nm}$ transparent ist und somit überwiegend Blauzapfen erregt, während beim Standard-(Weiß-)Muster-ERG alle Fotorezeptoren erregt werden. Das Absorptionsmaximum der Blauzapfen liegt bei $420 \mathrm{~nm}$ (Abb. 6, [35]). Der Bereich von 350 bis $450 \mathrm{~nm}$ erfasst jedoch auch einen Teil der Rot- und Grünzapfen sowie der Stäbchen. Demnach ergibt sich insgesamt ein höheres Potential als es der alleinige Blauzapfenanteil von 10\% bezogen auf die Anzahl aller Zapfen [30] erwarten lassen würde.

\subsubsection{Alterskorrelierte Verteilung des Blaulicht-Muster-ERGs}

Die Potentiale des Standard-P-ERGs betragen bei Probanden von 25 Jahren oder jünger 5,9 $\mu \mathrm{V}$. Bei Teilnehmern, die älter als 50 Jahren sind, liegen sie bei 3,8 $\mu \mathrm{V}$, was einer Herabsetzung auf $2 / 3$ des Wertes entspricht. Das Blaulicht-P-ERG erreicht bei den jüngeren Probanden eine Amplitude von 3,4 $\mu \mathrm{V}$, während es bei den älteren nur noch $0,9 \mu \mathrm{V}$ sind. Dies entspricht einer Verminderung auf $1 / 4$ der Amplitude im Vergleich zu den jüngeren Probanden. Dies bedeutet, dass mit steigendem Alter das Blaulicht-Muster-ERG deutlich stärker reduziert ist als das Standard-P-ERG. 


\section{Diskussion}

Begründet werden kann dies mit der Trübung der Linse. Die normale Linse ist transparent. Im Zuge des physiologischen Alterungsprozesses nimmt der Wassergehalt der Linse ab, sodass sie an Transparenz verliert und trüb wird. Die Farbe des Kerns hängt vom Alter der Katarakt $\mathrm{ab}$ und variiert von transparent - grau - graugelb - bernsteinfarben - braun zu schwarz [3; 8]. Eine trübe, gelb gefärbte Linse filtert insbesondere den blauen Anteil des Lichtes stärker [8], sodass weniger blaues Licht die Retina erreicht. Bei Verwendung eines Blaufilters wie in dieser Studie trifft nur blaues Licht zunächst auf die Linse, die je nach Ausprägung der Katarakt weniger und insbesondere kein dem Blauspektrum zugehöriges Licht passieren lässt. Daher ergeben sich dem Stadium der Linsentrübung entsprechend im Blaulicht-P-ERG nur minimale bis nicht mehr nachweisbare Amplituden.

Der Beginn der physiologischen Trübung der Linse ist individuell sehr unterschiedlich und wird subjektiv auf vielerlei Weise wahrgenommen. Keiner der Normalprobanden fühlt sich persönlich durch eine beginnende Katarakt eingeschränkt. Dennoch sind in den elektrophysiologischen Untersuchungen deutliche Amplitudenreduktionen vor allem bei Studienteilnehmern, die älter als 45 Jahre sind, erkennbar.

Demzufolge wurde eine Alterskorrelation der BW-M-ERG-Werte notwendig. Dies wurde mittels Regressionsanalyse umgesetzt, sodass Grenzwerte festgelegt werden konnten. In diese Analyse wurden jeweils beide Augen eines Probanden einzeln einbezogen. Die Schätzung der Standardabweichung aus diesen Daten könnte somit bei bedeutenden intraindividuellen Abhängigkeiten zu niedrig ausfallen. Eine Beschränkung der Regressionsanalyse auf ein zufällig ausgewähltes Auge pro Proband ergab aber keine wesentliche Änderung des geschätzten Referenzbereiches. In jedem Fall ist die Probandenzahl gering, sodass die Schätzung des Referenzbereichs als vorläufig zu betrachten ist. Mit einer besseren Schätzung aufgrund einer größeren Datenbasis lässt sich möglicherweise die Sensitivität des aus diesen altersabhängigen Schwellenwerten resultierenden diagnostischen Tests noch steigern.

Da diese Regressionsanalyse aus den Werten der 30 potentiell gesunden Studienteilnehmern gewonnen wurde, liegen folglich fast alle BW-M-ERG-Werte im errechneten Normbereich. Einer von den 60 gesunden Fällen lag statistisch gesehen unterhalb des Normbereiches (ID 19 OD). Ein Grund lässt sich hier bisher nicht finden. 


\section{Diskussion}

\subsubsection{Muster-ERG bei Kataraktpatienten}

Um nicht nur statistisch die Auswirkungen von Alter und Linsentrübung herauszurechnen, entsteht ein weiterer Studienzweig, der Kataraktpatienten mittels Standard-(weiß-) und Blaulicht-Muster-ERG untersucht.

Dass eine Trübung der optischen Medien elektrophysiologische Untersuchungen beeinflusst, ist lange bekannt und wurde auch bereits von der Arbeitsgruppe um P. Trüb genutzt. Sie untersuchten elf pseudophake Patienten, die einen Nachstar entwickelten, mittels PatternERG. In neun von elf Fällen ergaben sich gleichbleibende bis erniedrigte Amplituden. Der Annahme folgend, dass eine Medientrübung Amplitudenreduktionen im P-ERG auslöst, wandten sie das Muster-Elektroretinogramm als eine objektive Methode zur Erfassung des Nachstars an [43].

\subsubsection{Gesunde Kataraktpatienten}

Bei einer 53-jährigen Patientin (ID 50) bestehen präoperativ von Artefakten nicht mehr zu unterscheidende Ausschläge im Standard- sowie im Blaulicht-Muster-ERG der betroffenen Seite. Vier Monate nach Entfernung der trüben Linse und Ersatz durch eine Kunstlinse zeigt sich ein deutlicher Kurvenverlauf in beiden Muster-Elektroretinogrammen. Die Amplitude von 3,1 $\mu \mathrm{V}$ im Standard-P-ERG fällt nur auf 2,1 $\mu \mathrm{V}$ im Blaulicht-P-ERG, was mit 69,8\% über dem in dieser Studie bestimmten Normbereich für Blau-zu-Weiß-MusterElektroretinogramme liegt.

Die zweite Patientin (ID 51) wurde nur postoperativ untersucht. Die Amplitude des BlaulichtMuster-ERGs reduziert sich rechts auf 23,6\% und links auf 36,4\%. Unter Berücksichtigung des Patientenalters von 60 Jahren sind dies normale BW-M-ERG-Werte.

Diese Patientenbeispiele zeigen, dass bei einer Trübung der Linse das MusterElektroretinogramm vollständig erloschen sein kann. Nach einer operativen Kataraktentfernung sind jedoch wieder elektrophysiologische Potentiale messbar.

Diese Ergebnisse wurden in Bezug auf das herkömmliche P-ERG in ähnlicher Weise bereits von anderen Autoren beschrieben. Wördehoff und Kollegen veröffentlichten 2004 ihre Studie zum Einfluss der Katarakt auf das multifokale ERG sowohl prä- als auch postoperativ [44]. Sie postulierten, dass Trübungen der optischen Medien einen kleinen aber signifikanten Einfluss auf das multifokale ERG hätten und dass nach der Kataraktoperation die Amplitude im zentralen $4^{\circ}$-Radius ansteige. 


\section{Diskussion}

Ratanapakorn et al. untersuchten 2010 den Einfluss der Katarakt auf das Elektroretinogramm und kamen zu dem Ergebnis, dass die meisten der ERG-Kurven nach einer Kataraktoperation etwas größer waren als präoperativ, der Unterschied jedoch nicht statistisch signifikant war [45].

\subsubsection{Glaukomerkrankte Kataraktpatienten}

Die beiden von uns untersuchten Glaukompatientinnen (ID 60; ID 61) weisen nach erfolgreicher und komplikationsloser Kataraktoperation im Standard-P-ERG Amplituden von einerseits 4,9 $\mu \mathrm{V}$ und andererseits $2,1 \mu \mathrm{V}$ auf. Während der Wert der einen Patientin etwas hoch normal erscheint, reiht sich der Wert der zweiten Patientin in das Kollektiv der Glaukompatienten ohne Kataraktoperation ein.

Beim Blaulicht-Muster-ERG sind in beiden Fällen keine Amplituden messbar. Die Ausschläge sind vom stets vorhandenen Störungsrauschen nicht mehr zu unterscheiden, sodass sich annähernd eine Nulllinie ergibt. Dies entspricht unseren Erwartungen, da bei diesen Patienten das Glaukom bereits lange bekannt war und Sehnervenschäden in Form von Gesichtsfeldausfällen manifest waren, sodass auch eine Verbesserung der optischen Medien, wie der Ersatz der trüben Linse, keine Steigerung der P-ERG-Werte erzielen kann.

\subsubsection{Muster-ERG bei Glaukompatienten}

Das Standard-Muster-ERG ist mit durchschnittlich 2,3 $\mu \mathrm{V}$ deutlich geringer als in der Gruppe der gesunden Studienteilnehmer. Das Blaulicht-Muster-ERG ist in der Gruppe der Glaukompatienten meist vollständig erloschen oder nicht mehr messbar, da die minimalen Ausschläge von Artefakten nicht mehr zu unterscheiden sind.

Dies entspricht unserer Annahme, da Patienten mit einem manifesten Glaukom per definitionem an einer progredienten Sehnervenschädigung leiden [2]. Demnach sind bereits Schäden der Sehnervenzellen und ihren Verschaltungen aufgetreten und es werden somit nur noch wenige bis nicht mehr messbare Potentiale im Elektroretinogramm generiert. Insbesondere im Blaulicht-Muster-ERG macht sich dies bemerkbar, wo nur eine kleine Zellpopulation untersucht wird und Schäden frühzeitig auffallen. Folglich kann man postulieren, dass das Blau-P-ERG sensitiver als das Standard-P-ERG ist.

Bereits 1984 beschrieben Papst et al., dass bei Glaukompatienten mit Papillenschaden und Gesichtsfelddefekten eine signifikant erniedrigte Amplitude im P-ERG auffiel [21]. 


\section{Diskussion}

Ebenfalls Parisi und Mitarbeiter schrieben 2006, dass bei den 84 von ihnen untersuchten Offenwinkel-Glaukompatienten die P50- zu N95-Amplitude in allen 84 (100\%) Augen reduziert war, sodass sie postulierten: ,bei Patienten mit Offenwinkelglaukom hat im P-ERG die P50- zu N95-Amplitude eine Sensitivität von 100\%” [46].

\subsubsection{Muster-ERG bei Studienteilnehmern mit Glaukomverdacht}

Unter den Studienteilnehmern mit dem Verdacht auf ein Glaukom befanden sich demzufolge potentiell Gesunde sowie möglicherweise Patienten, die gerade ein Glaukom entwickeln. Demnach fielen die Ergebnisse der elektrophysiologischen Untersuchung sehr unterschiedlich aus.

Es erfolgt eine erneute Unterteilung dieser Gruppe in „eher Glaukom manifest“ und „eher kein Glaukom“. Das Entscheidungskriterium hierfür ist die aus dem klinischen Befund abgeleitete Therapieempfehlung, die laut ärztlichem Entlassungsbefund getroffen wurde.

Kritisch anzumerken ist hier, dass in 2/20 Fällen bereits zwei von drei Glaukom-Kriterien erfüllt waren (Gesichtsfeldausfall und auffällige Papillenexkavataion). In Zusammenschau aller Befundergebnisse konnte jedoch ein Glaukom daraufhin noch nicht gesichert diagnostiziert werden, sodass die Patienten zunächst als glaukomverdächtig eingestuft wurden.

In der Gruppe der „eher Glaukom-manifesten“ hatte laut unseren Grenzwerten einer von acht Teilnehmern ein „pathologisches“ Blaulicht-Muster-ERG. Von den sechs „eher kein Glaukom“-Patienten wurden alle als ,gesund“ identifiziert. Somit bestätigt dieses Verfahren anhand der Ober- und Untergrenzen alle potentiell gesunden Studienteilnehmer auch als gesund. „Eher Glaukom-manifeste“ Verdachtspatienten werden aber nicht in jedem Fall erkannt.

Ein Grund für diese schwache Korrelation scheint insbesondere der breite Referenzbereich zu sein. Vor allem im Alter schwanken die ERG-Werte sehr stark, was im jüngeren Kollektiv deutlich weniger zu beobachten ist. Demzufolge scheinen die Ober- und Untergrenzen insbesondere bei jüngeren Patienten sehr weit gefasst.

In Folge der Durchführung zahlreicher P-ERGs entwickelt sich ein Blick für normale Blau-PERGs oder scheinbar reduzierte Ergebnisse. Rechnerisch ergeben sich dann beispielsweise Reduktionen auf etwa 48\% des Standard-P-ERGs. Diese sind aber insbesondere bei jungen Menschen zu niedrig. Vergleicht man die Werte mit jenen der Gruppe der 20 bis 25-jährigen 


\section{Diskussion}

Normalprobanden, so reduziert sich bei ihnen das Blaulicht-Muster-ERG nur auf $58 \%$. So scheint es, dass zum Beispiel bei einer 23-jährigen „eher Glaukom manifesten“ Patientin (ID 201) ein BW-M-ERG von 47,1\% vielmehr zu niedrig ist, obwohl der Referenzbereich in diesem Alter zwischen 35,3\% und 82,6\% liegt. Der Mittelwert von 58,9\% zeigt hier deutlicher an, wo der Normwert liegen könnte. Falsch negative Aussagen über das BW-MERG sind die Folge.

Des weiteren kann der Fehler jedoch auch auf Seiten der getroffenen Therapieempfehlung liegen. Bei positiver Familienanamnese, zentral tief exkavierter Papille und grenzwertigen Augeninnendrücken (ID 203) ist jeder Ophthalmologe im Zweifelsfall eher geneigt, eine Therapie anzusetzen, um eventuelle Schäden abzuwenden. Das BW-M-ERG schien in diesem Fall völlig unauffällig, doch lässt sich zurzeit nicht sicher vorhersagen, ob dieser Patient ein Glaukom entwickeln wird oder nicht.

\subsection{Die optische Kohärenztomographie heute in der medizinischen Anwendung}

Das OCT zählt heute mit zu den wichtigsten bildgebenden Diagnostikgeräten in der Augenheilkunde und ist in vielen ophthalmologischen Praxen und Kliniken vorhanden. Neben dem Anwendungsgebiet bei Erkrankungen der Makula können mittels OCT Aussagen zur peripapillären Nervenfaserschichtdicke getroffen werden, welche zur Beurteilung eines Glaukomes von Bedeutung ist.

\subsubsection{Nervenfaserschichtdickenmessung mittels Stratus OCT bei gesunden Probanden}

In dieser Studie fallen aus der Auswertung der OCT-Ergebnisse drei Probanden aufgrund eines schrägen Sehnerveneintrittes (SSNE oder tilted disc syndrome) heraus. Oft sind es kurzsichtige Patienten, bei denen der Augenarzt bedingt durch den längeren Bulbus einen schrägen Eintritt des Sehnerven in das Auge beobachtet. Auch bei unseren Untersuchungen sind zwei (ID 18, ID 24) der drei Studienteilnehmer mit SSNE myop.

Die Papille erscheint hierbei oval und gekippt, sodass der untere Pol näher an der Makula liegt. Dies ist zunächst nicht krankhaft, erschwert jedoch die Papillendiagnostik [47]. Es führt bei der zirkulären Nervenfaserschichtdickenmessung mit dem OCT gehäuft zu niedrigen Dickenwerten, sodass im Vergleich mit der alterskorrelierten Datenbank die RNFL als zu niedrig eingestuft wird. Demzufolge werden viele Augen mit einem schrägen 


\section{Diskussion}

Sehnerveneintritt gemäß Nervenfaserschichtdickenmessung mittels Stratus OCT häufig als „erkrankt“ eingeordnet.

Sehr deutlich wird dieser Sachverhalt bei der Probandin mit der ID 18. Bei einer Myopie von $-6,75 \mathrm{dpt}$ rechts und -7,25 dpt links ist insbesondere superior die Nervenfaserschicht sowohl rechts als auch links mit $78 \mu \mathrm{m}$ im Stratus OCT absolut zu niedrig (Tabelle 1 und 6 im Anhang). Verglichen mit der altersbezogenen Datenbank liegen diese Werte im kritischen Bereich („rot“ im Kreisdiagramm/Sektor), folglich unter einem Prozent der Normalbevölkerung. Dies bedeutet, die alleinige Auswertung des OCTs würde den Ophthalmologen alarmieren und eventuell einen Nervenfaserschichtuntergang vermuten lassen.

Moschos et al. haben dies 2010 in ihrer Studie über das tilted disc syndrome untersucht [48]. Sie verglichen 21 Augen von 12 Patienten mit einem schrägen Sehnerveneintritt mit 40 Augen von 20 Probanden einer alters- und geschlechtsentsprechenden Kontrollgruppe mittels OCT und multifokalem Elektroretinogramm. Sie berichten über eine insbesondere superior signifikant erniedrigte retinale Nervenfaserschichtdicke und eine signifikant herabgesetzte Amplitude foveal und parafoveal im mfERG bei SSNE-Patienten. Nach Meinung dieser Arbeitsgruppe suggerieren diese Ergebnisse beim tilted disc syndrome eine Dysfunktion der Makula und des Sehnerven, die noch keine visuelle Beeinträchtigung herbei führten und mit anderen Untersuchungsmethoden noch nicht entdeckt wurden.

Bei zwei Normalprobanden ließ sich eine OCT-Untersuchung im Rahmen dieser Studie nicht mehr durchführen, somit entfielen vier weitere Augen für die endgültige Auswertung.

Alle weiteren Sehnervenfaserschichtdicken der 50 Augen der 25 gesunden Studienteilnehmer liegen gemäß der geräteeigenen normativen Datenbank im altersbezogenen Normbereich.

2010 veröffentlichten Mwanza und Mitarbeiter eine Studie der University of Miami zur Differenzierung zwischen gesunden und glaukomerkrankten Augen mittels Cirrus HD-OCT [49]. Sie führten die retinale Nervenfaserschichtdickenmessung sowie eine Analyse des Sehnervenkopfes an 73 Glaukompatienten und 146 gesunden altersentsprechenden Kontrollprobanden durch. Ihre durchschnittlich gemessenen retinalen Nervenfaserschichtdicken der gesunden $(90,21 \mu \mathrm{m} \pm 9,13)$ bzw. der mild glaukomerkrankten Augen $(70,21 \mu \mathrm{m} \pm 12,72)$ sind etwa $10 \%$ dünner als jene unserer Studienteilnehmer (gesund: 99,06 $\mu \mathrm{m} \pm$ 9,68; glaukomerkrankt: 76,15 $\mu \mathrm{m} \pm 14,74$ ). Erklärbar sind diese Unterschiede zum einen dadurch, dass es sich um unterschiedliche ethnische Bevölkerungsgruppen handelt. Zum anderen kann das gewählte Untersuchungsverfahren 


\section{Diskussion}

ebenfalls Unterschiede herbeiführen. Das hier verwendete HD-OCT ist ein Spectral-Domain OCT, eine Weiterentwicklung des von uns benutzten Stratus OCTs, einem Time-Domain OCT. Die axiale Auflösung des SD-OCTs ist mit 5-7 $\mu \mathrm{m}$ genauer als jene des TD-OCTs mit $10 \mu \mathrm{m}$ [18], sodass sich bereits hier Unterschiede ergeben können. Dennoch sind die Differenzen vergleichbar, sodass die von uns gemessenen Ergebnisse durchaus plausibel erscheinen.

\subsubsection{Nervenfaserschichtdickenmessung mittels Stratus OCT bei Glaukompatienten}

Die durchschnittliche retinale Nervenfaserschichtdicke bei Patienten mit einem Glaukom ist in dieser Studie in 46\% der Fälle grenzwertig beziehungsweise deutlich erniedrigt.

Eine weitere Studie zur Messung der RNFL wurde beispielsweise von Mansoori und Mitarbeitern 2010 veröffentlicht [50]. Sie quantifizierten die RNFL in 66 normalen, 55 okulär hypertensiven (OHT) und 51 glaukomatösen Augen in der indischen Bevölkerung. Die durchschnittliche Nervenfaserschicht war mit 93,45 $\mu \mathrm{m}( \pm 16,9 \mu \mathrm{m})$ in glaukomatösen Augen signifikant erniedrigt im Vergleich zu 110,09 $\mu \mathrm{m}( \pm 10,9 \mu \mathrm{m})$ bei Patienten mit OHT und $112,48 \mu \mathrm{m}( \pm 6,8 \mu \mathrm{m})$ in gesunden Augen. Sie erkannten mittels Spectral-Domain OCT deutliche Unterschiede zwischen glaukomatösen und normalen Augen. Limitiert sahen sie jedoch diese Untersuchungsmethode in der Differenzierung zwischen gesunden Augen und solchen mit okulärem Hochdruck, da es hier häufig zu Überschneidungen kam.

Verglichen mit den im Rahmen unserer Studie gewonnenen Mittelwerten, sind die Nervenfaserschichtdicken der indischen Bevölkerung im Schnitt etwa $15 \mu \mathrm{m}$ dicker. Dies kann auch hier populationsbedingt der Fall sein. So berichteten bereits Samarawickrama und Kollegen in einer australischen Studie von 2010 über ethnische Unterschiede zwischen europäischen kaukasischen und ostasiatischen Kindern bezüglich der retinalen Nervenfaserschichtdicke [51]. Sie fanden heraus, dass ostasiatische Kinder im Schnitt dickere RNFLs und eine größere Cup-disc-ratio aufwiesen als kaukasische Kinder.

Mansoori verwendete ebenfalls wie Mwanza und Kollegen das SD-OCT. Die Zahlenwerte sind mit unserem Stratus OCT nicht zu 100\% identisch. Eine Tendenz stellt sich jedoch dar: Glaukompatienten können eine reduzierte Nervenfaserschichtdicke aufweisen. 


\section{Diskussion}

\subsubsection{Nervenfaserschichtdickenmessung mittels Stratus OCT bei Studienteilnehmern mit Glaukomverdacht}

Wie die Muster-ERG-Messungen zeigen auch die Nervenfaserschichtdicken in der Gruppe IV sehr unterschiedliche Ergebnisse, sodass die Gruppeneinteilung in „eher Glaukom manifest“ und „eher kein Glaukom“ auch hier beibehalten wird. Dadurch zeichnet sich ein deutlicher Trend ab: In der Gruppe der „eher Glaukom manifesten“ Patienten werden neun von 26 Augen als „grenzwertig“ oder „pathologisch“ bewertet. In der „eher gesunden“ Gruppe sind alle 14 Augen der sieben Teilnehmer im Grünbereich der RNFL-Messung und werden somit laut OCT als ,gesund“ bestätigt.

Aufschlussreich ist hierzu im Vergleich die Studie von 2010 von Kim et al. [52] aus Korea. Sie untersuchten 60 Augen von Patienten mit präperimetrischem Glaukom und 60 gesunde Augen mittels Laser-Polarimetrie (GDx VCC) und optischer Kohärenztomographie (Stratus OCT) bezüglich der Nervenfaserschichtdicke. Zur Gegenüberstellung mit unseren Resultaten sind jene der OCT interessant. Im Ergebnis dieser Arbeitsgruppe erreichten Patientenaugen mit präperimetrischem Glaukom Dickenwerte von 86,43 $\mu \mathrm{m}( \pm 20,49 \mu \mathrm{m})$ und gesunde Augen 106,61 $\mu \mathrm{m}( \pm 9,57 \mu \mathrm{m})$. Wie diese Gruppe das präperimetrische Glaukom konkret ermittelt, ist aus der Publikation nicht zu entnehmen. Aller Wahrscheinlichkeit nach wird es jedoch ein Glaukom sein, was unserem „Verdachtsglaukom“ ähnlich ist: keine Gesichtsfeldausfälle, aber auffällige Befunde der Papille oder im Tagesdruckprofil. Daher sind die Werte auch denen in unserer Studie gefundenen sehr ähnlich. Die Werte der gesunden Teilnehmer liegen nur $6 \mu \mathrm{m}$ über unserem Mittelwert und die der präperimetrischen Glaukompatienten entsprechen etwa unseren „eher Glaukom erkrankten“ Nervenfaserschichtdicken mit $88 \mu \mathrm{m}$. Folglich kann sich bei der Entwicklung eines Glaukoms wie auch bei einem bereits manifesten Glaukom ein Sehnervenschaden in der Nervenfaserschichtdickenmessung zeigen. Er ist jedoch nicht in jedem Fall vorhanden.

\section{3 Übereinstimmung der beiden Verfahren}

Beim Vergleich der Ergebnisse des Blaulicht-Muster-Elektroretinogramms und der optischen Kohärenztomographie können übereinstimmende Aussagen z.B. im Fall der Patientin (ID 206) getroffen werden, bei der ein komplett erloschenes Blaulicht-P-ERG und eine stark erniedrigte Nervenfaserschichtdicke (OS) auffallen. Gleichzeitig imponiert auch der Fall mit 


\section{Diskussion}

der ID 218 bezeichneten Patientin mit großer Amplitude im B-P-ERG und normaler Nervenfaserschichtdicke.

Dennoch finden sich widersprüchliche Daten, wie diejenigen des Patienten (ID 202) mit einer hoch normalen Relation des Blau- zu Weiß-Muster-ERGs (OD 46,4\%; OS 50,0\%) und einer laut OCT deutlich erniedrigten Nervenfaserschichtdicke (OD 76,54 $\mu \mathrm{m}$; OS 80,65 $\mu \mathrm{m}$ ). Bei diesem jungen Patient bestand der Verdacht auf ein Glaukom. Bei ihm waren seit fünf Wochen unklare Gesichtsfelddefekte bekannt, er hatte einen grenzwertig erhöhten Augeninnendruck (OD $22 \mathrm{mmHg}$; OS $21 \mathrm{mmHg}$ ) und wurde zunächst antiglaukomatös therapiert. Da die P-ERG Untersuchung bereits im Februar 2009 erfolgte, konnte dieser Patient auch im Verlauf beobachtet werden. Laut Arztbriefen wurde er aufgrund von rezidivierenden Gesichtsfeldausfällen weitere dreimal stationär aufgenommen. Neurologische Untersuchungen ergaben keinen Hinweis auf ZNS-Erkrankungen. Molekulargenetische Untersuchungen schlossen die hereditäre Leber'sche Optikusatrophie aus. Ein juveniles Glaukom wurde letztendlich aufgrund untypischen Verlaufs ausgeschlossen, wie es das Muster-ERG anfangs bereits vermuten ließ. Abschließend konnte dem Patienten eine Simulation aus beruflichen Gründen nachgewiesen werden. Sicher ist dieser Patient kein gewöhnlicher Studienteilnehmer, aber er entspricht dem klinischen Alltag. Anfangs bestand nur der auffällige Befund in der Perimetrie und die Patientenanamnese. Es war Aufgabe des Augenarztes $\mathrm{zu}$ entscheiden, ob es sich um ein beginnendes Glaukom oder Schwerwiegenderes handelte. Verständlicherweise geht man zunächst den sicheren Weg und therapiert frühzeitig trotz eines weiteren diagnostischen Werdeganges. Das Muster-ERG hätte hier als ein Diagnosebaustein gewertet werden können, um eine Pathologie auszuschließen.

Zum Vergleich des Blaulicht-Muster-ERGs mit dem Stratus OCT wird eine Kreuztabelle erstellt (siehe Ergebnisteil Tabelle 15). Demnach kommen beide Verfahren in 87\% der Fälle (71 von 82) zur gleichen Entscheidung. In etwa 80\% (66 von 82) der Fälle wurden die untersuchten Augen anhand beider Verfahren übereinstimmend als ,gesund“ bewertet. Somit haben beide Tests in 43 von 44 Fällen potenziell gesunde Augen als gesund bestätigt, was einer hohen Spezifität entspricht, und neun von zehn Verdachtspatienten in der Gruppe „eher kein Glaukom“ zusätzlich bestätigt. Umgekehrt wurden fünf Augen von beiden Methoden als pathologisch eingeschätzt, von denen vier Augen bereits ein manifestes Glaukom hatten und das fünfte zur Gruppe der „eher Glaukom manifesten“ gehörte. 


\section{Diskussion}

Die Gegenüberstellung der ERG-Ergebnisse und der Ergebnisse der RNFL-Messungen in Bezug zum klinischen Befund (siehe Ergebnisteil Tabelle 22) erlaubt eine Schätzung von Sensitivität und Spezifität der beiden Verfahren. Insbesondere für die Sensitivität ist jedoch kritisch anzumerken, dass wir nur eine geringe Fallzahl betrachten und die Schätzung somit unpräzise ist.

Die ERG-Untersuchung hat mit 97,7\% ähnlich wie die OCT-Messung mit 100\% eine hohe geschätzte Spezifität. Welche der beiden Methoden die höhere Spezifität hat, konnten wir dennoch nicht feststellen, da nur in einem einzigen Fall beide Verfahren zu einem unterschiedlichen Ergebnis kamen.

Um ein rechnerisches Maß für die Übereinstimmung der beiden Verfahren zu finden, wird Cohens Kappa-Koeffizient bestimmt. Die Stärke einer Übereinstimmung nimmt mit steigendem $\kappa$ (von null bis eins) zu. Die Tabelle 24 stellt eine grobe Interpretationshilfe dar. Tatsächlich nimmt die Übereinstimmung mit steigendem Kappa kontinuierlich zu.

Tab. 24: Bewertung von $\kappa[39 ; 53]$

\begin{tabular}{|c|c|}
\hline$\kappa$ & Übereinstimmung \\
\hline$<0$ & Schlecht (poor) \\
$0-0,20$ & Etwas (slight) \\
$0,21-0,40$ & Ausreichend (fair) \\
$0,41-0,60$ & Mittelmäßig (moderate) \\
$0,61-0,80$ & Beachtlich (substantial) \\
$0,81-1,00$ & (Fast) Vollkommen ((almost) perfect) \\
\hline
\end{tabular}

Ein Cohens Kappa-Koeffizient von 0,4 weist auf eine ausreichende an der Grenze zur moderaten Übereinstimmung beider Verfahren hin [39; 53]. Das 95\%-Konfidenzintervall veranschaulicht dagegen die Ungenauigkeit dieser Schätzung: Ein $\kappa$ von 0,1 beschreibt etwas Übereinstimmung, wohingegen eines von 0,7 eine beachtliche Übereinstimmung bedeutet [39; 53]. Demnach können wir mit 95\% Konfidenz sagen, dass mindestens etwas Übereinstimmung über die zufällig erwartete hinaus besteht.

In den untersuchten Daten sind von den 82 eingeschlossenen Fällen nur zehn Augen gemäß OCT und elf laut BW-M-ERG als pathologisch klassifiziert worden. Daraus ergeben sich nur geringe Randsummen. Dies ist für die Berechnung des Cohen's Kappa-Koeffizienten von großer Bedeutung und führt in dieser Studie dazu, dass die Schätzung des $\kappa$ ungenau wird. Demnach wird ein gesunder Patient in beiden Verfahren meist als „gesund“ erkannt, ob 


\section{Diskussion}

jedoch ein Erkrankter gleichfalls nach beiden Methoden als „pathologisch“ erkannt wird, muss präziser in einer Studie untersucht werden, an der Glaukompatienten in großer Zahl teilnehmen.

Auch Falsini und Mitarbeiter prüften 2008 Struktur-Funktionsbeziehung bei Patienten mit okulären Hypertensionen und Glaukom [24]. Sie untersuchten die retinale Nervenfaserschichtdicke und die retinale Ganglienzellaktivität von 31 OHT-, 34 Glaukompatienten und 16 gesunden Kontrollprobanden mittels Stratus OCT und MusterERG. Im Ergebnis sahen sie erniedrigte Amplituden im P-ERG sowie ausgedünnte RNFLs bei OHT- und Glaukompatienten. Es bestand eine positive Korrelation zwischen beiden Untersuchungsverfahren bei Patienten mit einem manifesten Glaukom. Bei Personen mit erhöhtem Augeninnendruck war die RNFL jedoch meist normal und nur die P-ERGAmplitude erniedrigt, sodass sie hier den Früherkennungswert des MusterElektroretinogramms bestätigt sahen.

In unserer Studie wurde weniger die Überlegenheit eines Verfahrens gegenüber dem anderen deutlich, sondern vielmehr die verbesserte Aussagekraft beider Messmethoden in der Kombination. Die Verwendung des Blaufilters war jedoch ein entscheidender Faktor in der Methode des Muster-Elektroretinogramms. Zwar sind auch bereits die Potentiale im Standard-P-ERG deutlich erniedrigt, doch ist eine viel augenscheinlichere Reduktion bei Verwendung des optischen Filters sichtbar geworden. Während im Standard-P-ERG auch Glaukompatienten oftmals noch sichtbare Kurvenverläufe zeigten, was für die Durchführbarkeit der Methode spricht, waren im Blaulicht-Muster-ERG insbesondere die Potentiale der Patienten mit manifestem Glaukom erloschen. Wenn man davon ausgeht, dass mit diesem Verfahren tatsächlich eine Nervenpopulation untersucht wird, hier die Blauzapfen und das koniozelluläre Ganglienzellsystem, die aufgrund ihrer geringen Anzahl bei Schäden zuerst betroffen ist, kann die These der reduzierten Redundanz bestätigt werden.

Kritisch betrachtet werden muss hier allerdings die extreme Empfindlichkeit des MusterElektroretinogramms gegen Störfaktoren, was sich in falsch zu niedrigen Potentialen äußert. Ein trockenes Auge ist hierbei ebenso hinderlich wie zuviel Tränenfluss durch eine nicht ausreichende Analgesie der Hornhaut. Ein unzureichend motivierter oder unaufmerksamer Proband wird schlechtere Ergebnisse liefern, als wenn er ausgeruht und kooperativ zu einem späteren Zeitpunkt untersucht wird. Ebenfalls ist die bestmögliche Refraktion für optimale Resultate unerlässlich. Das Kontrastempfinden ist früh eingeschränkt und so zeigen sich falsch-niedrige Amplituden im ERG, die nach Visuskorrektur reversibel sind. Möglichst 


\section{Diskussion}

ungetrübte optische Medien sind eine wichtige Voraussetzungen für diese Untersuchung. Daher kommt der Katarakt eine bedeutende Rolle zu, wie bereits aufgeführt wurde. Auch durch die Katarakt war das Standard-P-ERG erniedrigt, das B-P-ERG hingegen noch deutlicher. Als ergänzende Überlegung kann man diskutieren, ob die Katarakt ein natürlicher Schutzmechanismus des Auges ist. Viele Studien berichten über Schäden der Makula und der Netzhaut durch ultraviolettes oder kurzwelliges $(<500 \mathrm{~nm})$ Licht [54; 55].

Studien wie die von Margrain und Mitarbeitern untersuchten, ob Blaulichtfilter Schutz vor der altersbedingten Makuladegeneration bieten [56]. Sie zeigten, dass kurzwelliges Licht oxidativen Stress erzeugt, der zur Entwicklung der AMD beiträgt. Im Ergebnis konnten sie jedoch noch nicht beweisen, sondern nur vermuten, dass blaues Licht ein Risikofaktor für die Entstehung der AMD ist.

Aus dieser Überlegung heraus gibt es seit den 1990iger Jahren UV-absorbierende Intraokularlinsen, die in der Kataraktchirurgie zum Einsatz kommen.

Henderson et al. haben 2010 Studien von 1962 bis 2009 hinsichtlich von Beeinträchtigungen dieser Intraokularlinsen verglichen. Sie schreiben, dass der Nutzen der UV-Blockade in Bezug auf die AMD weiterhin unklar bleibt, sich diese Linsen jedoch nicht nachteilig auf Sehstärke, Farbwahrnehmung und Kontrastempfinden auswirken [57].

Folglich könnte man auch bei kataraktoperierten Patienten das Verfahren des BlaulichtMuster-ERGs anwenden. Nach Entfernung der trüben Linse ist ein normales Muster-ERG zu erwarten. Ist dies nicht der Fall und bestehen weitere Verdachtspunkte für ein Glaukom, muss dies als Diagnose in Erwägung gezogen werden. Patienten mit ihrer natürlichen Linse sind subjektiv nicht immer durch die Katarakt eingeschränkt. Doch sind sie laut unseren Studienergebnissen etwa ab dem 56. Lebensjahr nicht mehr sicher von Glaukompatienten zu unterscheiden. Sollten demnach gesunde ältere Patienten reduzierte Potentiale im BlaulichtMuster-ERG aufweisen, ist dies nicht zwangsläufig hinweisend auf ein Glaukom.

Das Alter oder vielmehr die Trübung der optischen Medien, wie auch die benötigte bestmögliche Mitarbeit der Patienten bedeuten limitierende Faktoren dieses elektrophysiologischen Verfahrens. Erst unter für das visuelle System des Patienten optimalen Bedingungen liefert das B-P-ERG valide und verlässliche Ergebnisse.

Der Vorteil dieser Untersuchungsmethode ist, dass sie schnell und kostengünstig durchführbar ist. Eine elektrophysiologische Untersuchungseinheit steht in sämtlichen Kliniken aber auch in zahlreichen Augenarztpraxen zur Verfügung. Diese Untersuchungseinheit muss lediglich um ein optisches Filter mit einem 


\section{Diskussion}

Transmissionsspektrum im Blaubereich ergänzt werden. Jede elektrophysiologisch ausgebildete Fachkraft kann damit ein Blaulicht-Muster-ERG aufzeichnen lassen und dies zu dem gewohnten Standard-P-ERG in Beziehung setzen. Die von uns erstellte Tabelle (Tabelle 7 im Anhang) gibt Richtwerte für gesunde BW-M-ERG-Potentiale.

In dieser Arbeit wurde nur ein kleines Kollektiv untersucht. Wenn in weiteren Studien mit größeren Fallzahlen und längerer Beobachtungsdauer gezeigt werden könnte, dass Patienten mit Glaukomverdacht, die auffällig im BW-M-ERG sind, tatsächlich ein Glaukom entwickeln, könnte dieses Verfahren eine neue, einfach durchzuführende Methode zur Früherkennung des Glaukoms im präperimetrischen Stadium werden. 


\section{$5 \quad$ Zusammenfassung}

Diese Studie untersuchte an insgesamt 70 Teilnehmern das neue Verfahren des BlaulichtMuster-Elektroretinogramms zum Glaukomscrenning. Dies misst die Funktion der koniozellulären Ganglienzellen, ein System retinaler Ganglienzellen, welches nur einen geringen Anteil an der Netzhaut ausmacht und somit bei Nervenzellschäden frühzeitig Defekte aufweist. Anhand der elektrophysiologischen Potentiale von 30 gesunden Studienteilnehmern wurden alterskorrigierte Normwerte geschätzt, da die physiologische Linsentrübung durchschnittlich ab dem Alter von 56 Jahren eine sichere Differenzierung zwischen gesund und glaukomverdächtig verhinderte. Mittels dieser Grenzwerte wurden Katarakt-, Glaukom- und Glaukomverdachtspatienten als „gesund“ oder „erkrankt“ klassifiziert. Diese Einteilung wurde mit jenen Ergebnissen der optischen Kohärenztomographie verglichen, einem Verfahren, welches die peripapilläre Netzhautdicke misst, die bei Glaukompatienten häufig reduziert ist. Eine Übereinstimmung der Endresultate wurde in $87 \%$ der Fälle (71 von 82) festgestellt. In beiden Verfahren werden Gesunde meist auch als ,gesund“ bestätigt und manifest Glaukomerkrankte ebenfalls korrekt als ,erkrankt“ erkannt. Jedoch fallen nicht alle „eher Glaukom manifesten“ Verdachtspatienten immer durch pathologische BW-M-ERGs oder reduzierte Nervenfaserschichtdicken im OCT auf. Entweder wurde die Diagnose „Glaukom“ fälschlich getroffen und dieser Patient wird in Wirklichkeit nie ein Glaukom entwickeln oder er befindet sich noch in einem so frühen Stadium der Erkrankung, dass der Nervenzellschaden weder mittels OCT noch mit dem P-ERG gemessen werden kann.

Das Blaulicht-Muster-ERG ist eine einfache und kostengünstige Methode als zusätzliches Verfahren in der Glaukomdiagnostik. Es kann einen Glaukomverdacht erhärten oder ihn abschwächen. In Kombination mit dem OCT hat es eine valide Aussagekraft. Somit kann das Muster-Elektroretinogramm mit einem Blaulichtfilter schnell und günstig vor allem bei Verdachtsfällen hinzugezogen werden. Jedoch müssen weitere Studien folgen, um die alterskorrigierten Normwerte enger einzugrenzen und weitere mögliche Einflussfaktoren zu überprüfen. 


\section{Literaturverzeichnis}

\section{Literaturverzeichnis}

1. Gemoll W, ed. Griechisch-Deutsches Schul- und Handwörterbuch. Vol. 10. Auflage. München: Oldenbourg Schulbuchverlag, 2006.

2. Pfeiffer N. Glaukom und okuläre Hypertension. Vol. 2. Stuttgart: Georg Thieme Verlag KG, 2005.

3. Kanski JJ. Klinische Ophthalmologie. Vol. 5. München: Elsevier, 2004.

4. Foster PJ, Buhrmann R, Quigley HA, Johnson GJ. The definition and classification of glaucoma in prevalence surveys. Br J Ophthalmol 2002;86(2):238-42.

5. Quigley HA, Dunkelberger GR, Green WR. Retinal ganglion cell atrophy correlated with automated perimetry in human eyes with glaucoma. Am J Ophthalmol 1989;107(5):453-64.

6. Kitazawa Y, Horie T, Aoki S, et al. Untreated ocular hypertension. A long-term prospective study. Arch Ophthalmol 1977;95(7):1180-4.

7. Sharts-Hopko NC, Glynn-Milley C. Primary open-angle glaucoma. Am J Nurs 2009;109(2):40-7; quiz 8.

8. Grehn F. Augenheilkunde. Vol. 29. Heidelberg: Springer Medizin Verlag, 2006.

9. Klein BE, Klein R, Sponsel WE, et al. Prevalence of glaucoma. The Beaver Dam Eye Study. Ophthalmology 1992;99(10):1499-504.

10. Quigley HA, Broman AT. The number of people with glaucoma worldwide in 2010 and 2020. Br J Ophthalmol 2006;90(3):262-7.

11. Kass MA, Heuer DK, Higginbotham EJ, et al. The Ocular Hypertension Treatment Study: a randomized trial determines that topical ocular hypotensive medication delays or prevents the onset of primary open-angle glaucoma. Arch Ophthalmol 2002;120(6):701-13; discussion 829-30.

12. Plange N, Kaup M, Daneljan L, et al. 24-h blood pressure monitoring in normal tension glaucoma: night-time blood pressure variability. J Hum Hypertens 2006;20(2):137-42.

13. Berufsverband der Augenärzte Deutschlands e.V. DOGeV. Leitlinie Nr. 15 a. 2006 www.augeninfo.de/leit/leit15a.pdf

14. Berufsverband der Augenärzte Deutschlands e.V. DOGeV. Leitlinie Nr. 15 c. 2006 www.augeninfo.de/leit/leit15c.pdf 


\section{Literaturverzeichnis}

15. Huang D, Swanson EA, Lin CP, et al. Optical coherence tomography. Science 1991;254(5035):1178-81.

16. Alper J. OCT: images of coherence. Science 1993;261(5121):555.

17. Schuman JS, Hee MR, Arya AV, et al. Optical coherence tomography: a new tool for glaucoma diagnosis. Curr Opin Ophthalmol 1995;6(2):89-95.

18. Schuman JS. Spectral domain optical coherence tomography for glaucoma (an AOS thesis). Trans Am Ophthalmol Soc 2008;106:426-58.

19. Gyatsho J, Kaushik S, Gupta A, et al. Retinal nerve fiber layer thickness in normal, ocular hypertensive, and glaucomatous Indian eyes: an optical coherence tomography study. J Glaucoma 2008;17(2):122-7.

20. May JG, Ralston JV, Reed JL, Van Dyk HJ. Loss in pattern-elicited electroretinograms in optic nerve dysfunction. Am J Ophthalmol 1982;93(4):418-22.

21. Papst N, Bopp M, Schnaudigel OE. Pattern electroretinogram and visually evoked cortical potentials in glaucoma. Graefes Arch Clin Exp Ophthalmol 1984;222(1):2933.

22. Berninger TA, Arden GB. The pattern electroretinogram. Eye (Lond) 1988;2 Suppl:S257-83.

23. North RV, Jones A, Drasdo N, et al. Electrophysiological evidence for early functional damage in glaucoma and ocular hypertension. Invest Ophthalmol Vis Sci 2009.

24. Falsini B, Marangoni D, Salgarello T, et al. Structure-function relationship in ocular hypertension and glaucoma: interindividual and interocular analysis by OCT and pattern ERG. Graefes Arch Clin Exp Ophthalmol 2008;246:1153-1162.

25. Bach M, Unsoeld AS, Philippin H, et al. Pattern ERG as an early glaucoma indicator in ocular hypertension: a long-term, prospective study. Invest Ophthalmol Vis Sci 2006;47(11):4881-7.

26. Holder GE, Brigell MG, Hawlina M, et al. ISCEV standard for clinical pattern electroretinography--2007 update. Doc Ophthalmol 2007;114(3):111-6.

27. RetinaScience. Muster-Elektroretinogramm. 2009.

28. Bach M, Hoffmann MB. Update on the pattern electroretinogram in glaucoma. Optom Vis Sci 2008;85(6):386-95.

29. Erb C, Gobel K. Functional glaucoma diagnosis. Ophthalmologe 2009;106(4):375-85; quiz 86.

30. Gouras P. The role of S-cones in human vision. Doc Ophthalmol 2003;106(1):5-11. 


\section{Literaturverzeichnis}

31. Spry PG, Johnson CA, Mansberger SL, Cioffi GA. Psychophysical investigation of ganglion cell loss in early glaucoma. J Glaucoma 2005;14(1):11-9.

32. Kurtenbach A, Flogel W, Erb C. Anomaloscope matches in patients with diabetes mellitus. Graefes Arch Clin Exp Ophthalmol 2002;240(2):79-84.

33. Malukiewicz G, Lesiewska-Junk H, Kazmierczak K. Changes in the colour vision and contrast sensitivity in diabetic patients without retinopathy. Klin Oczna 2009;111(79):221-3.

34. Greenstein VC, Hood DC, Ritch R, et al. S (blue) cone pathway vulnerability in retinitis pigmentosa, diabetes and glaucoma. Invest Ophthalmol Vis Sci 1989;30(8):1732-7.

35. H.J.A. BJKaD. Visual pigments of rods and cones in a human retina. J. Physiol. 1980;298:501-11.

36. Arden G, Wolf J, Berninger T, et al. S-cone ERGs elicited by a simple technique in normals and in tritanopes. Vision Res 1999;39(3):641-50.

37. Ihaka R. G, R. A language for data analysis and graphics. J Comput Graph Stat 1996;5(3):299-314.

38. Cohen J. A Coefficient of Agreement for Nominal Scales. Educ Psychol Meas 1960;20:37-46.

39. Lothar Sachs JH. Angewandte Statistik. Vol. 13: Springer, 2009.

40. Nowacka B, Lubinski W, Karczewicz D. Ophthalmological and electrophysiological features of Parkinson's disease. Klin Oczna 2010;112(7-9):247-52.

41. Krasodomska K, Lubinski W, Potemkowski A, Honczarenko K. Pattern electroretinogram (PERG) and pattern visual evoked potential (PVEP) in the early stages of Alzheimer's disease. Doc Ophthalmol 2010;121(2):111-21.

42. Bubl E, Kern E, Ebert D, et al. Seeing gray when feeling blue? Depression can be measured in the eye of the diseased. Biol Psychiatry 2010;68(2):205-8.

43. Trub P, Wildberger H, Hendrickson P, Robert Y. Discrete media clouding and its effect of contrast transfer coefficients, the pattern ERG and contrast sensitivity. Klin Monbl Augenheilkd 1992;200(5):444-6.

44. Wordehoff UV, Palmowski AM, Heinemann-Vernaleken B, et al. Influence of cataract on the multifocal ERG recording--a pre- and postoperative comparison. Doc Ophthalmol 2004;108(1):67-75. 


\section{Literaturverzeichnis}

45. Ratanapakorn T, Patarakittam T, Sinawat S, et al. Effect of cataract on electroretinographic response. J Med Assoc Thai 2010;93(10):1196-9.

46. Parisi V, Miglior S, Manni G, et al. Clinical ability of pattern electroretinograms and visual evoked potentials in detecting visual dysfunction in ocular hypertension and glaucoma. Ophthalmology 2006;113(2):216-28.

47. Park CY, Kim YT, Kee C. Evaluation of the influence of tilt of optic disc on the measurement of optic disc variables obtained by optical coherence tomography and confocal scanning laser ophthalmoscopy. J Glaucoma 2005;14(3):210-4.

48. Moschos MM, Triglianos A, Rotsos T, et al. Tilted disc syndrome: an OCT and mfERG study. Doc Ophthalmol 2009;119(1):23-8.

49. Mwanza JC, Oakley JD, Budenz DL, Anderson DR. Ability of Cirrus HD-OCT Optic Nerve Head Parameters to Discriminate Normal from Glaucomatous Eyes. Ophthalmology 2011;118:241-248.

50. Mansoori T, Viswanath K, Balakrishna N. Quantification of Retinal Nerve Fiber Layer Thickness in Normal Eyes, Eyes with Ocular Hypertension, and Glaucomatous Eyes with SD-OCT. Ophthalmic Surg Lasers Imaging 2010;41(6):S50-7.

51. Samarawickrama C, Wang JJ, Huynh SC, et al. Ethnic differences in optic nerve head and retinal nerve fibre layer thickness parameters in children. $\mathrm{Br} \mathrm{J}$ Ophthalmol 2010;94(7):871-6.

52. Kim HG, Heo H, Park SW. Comparison of Scanning Laser Polarimetry and Optical Coherence Tomography in Preperimetric Glaucoma. Optom Vis Sci 2011;88:124-129.

53. Landis JR, Koch GG. The measurement of observer agreement for categorical data. Biometrics 1977;33(1):159-74.

54. Korner-Stiefbold U. Age-related macular degeneration (AMD)-therapeutic possibilities and new approaches. Ther Umsch 2001;58(1):28-35.

55. Wierzbowska J, Figurska M, Stankiewicz A, Sierdzinski J. Risk factors in age-related macular degeneration and glaucoma--own observations. Klin Oczna 2008;110(1012):370-4.

56. Margrain TH, Boulton M, Marshall J, Sliney DH. Do blue light filters confer protection against age-related macular degeneration? Prog Retin Eye Res 2004;23(5):523-31.

57. Henderson BA, Grimes KJ. Blue-blocking IOLs: a complete review of the literature. Surv Ophthalmol 2010;55(3):284-9. 


\section{Anhang}

RNFL THICKNESS AVERAGE ANALYSIS

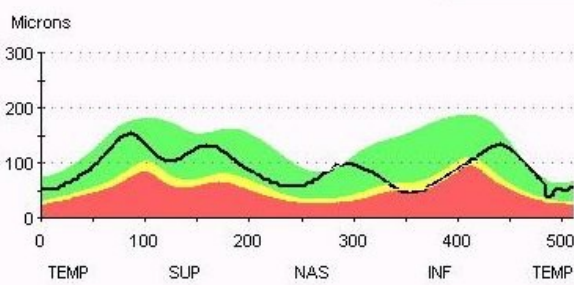

OD
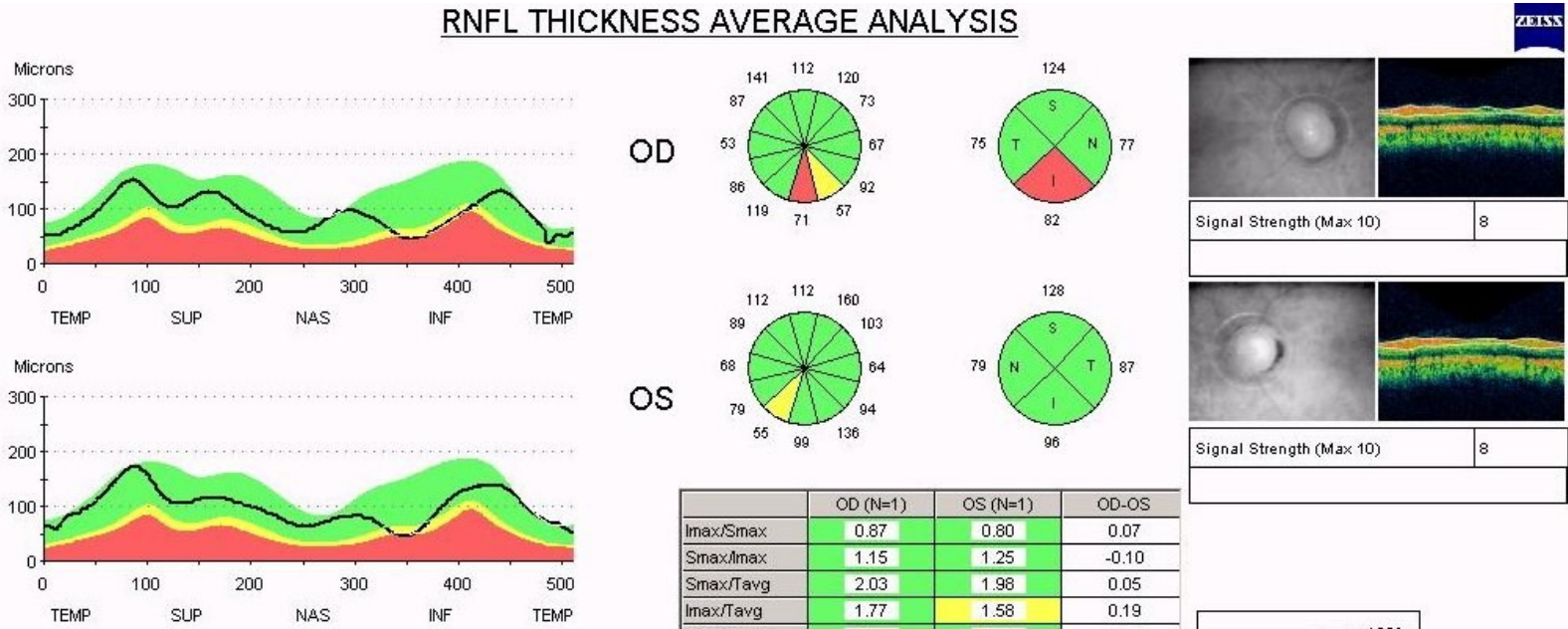

OS
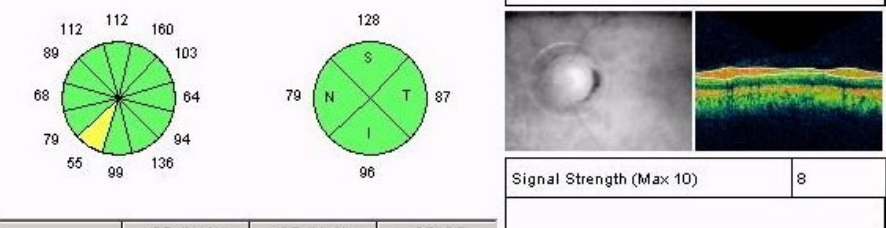

\begin{tabular}{|c|c|c|c|}
\hline & $\mathrm{OD}(\mathrm{N}=1)$ & $O S(N=1)$ & OD-OS \\
\hline Imaxisinax & 0.87 & 0.80 & 0.07 \\
\hline SmaxAlnax & 1.15 & 1.25 & -0.10 \\
\hline Smaxiavg & 2.03 & 1.98 & 0.05 \\
\hline Imax/Tavg & 1.77 & 1.58 & 0.19 \\
\hline SinaxNavg & 1.98 & 2.20 & -0.21 \\
\hline Max-Min & 115.00 & 125.00 & -10.00 \\
\hline Smax & 153.00 & 173.00 & -20.00 \\
\hline Inax & 133.00 & 138.00 & -5.00 \\
\hline Savg & 124.00 & 128.00 & -4.00 \\
\hline lavg & 82.00 & 96.00 & -14.00 \\
\hline Avg.Thickness & 89.75 & 97.46 & -7.71 \\
\hline \multicolumn{4}{|c|}{ Patient/Scan Information } \\
\hline Scan Type & \multicolumn{3}{|c|}{ RNFL Thickness (3.4) } \\
\hline Scan Date & \multicolumn{3}{|l|}{$2 / 5 / 2008$} \\
\hline Scan Length & \multicolumn{3}{|l|}{$10.87 \mathrm{~mm}$} \\
\hline
\end{tabular}
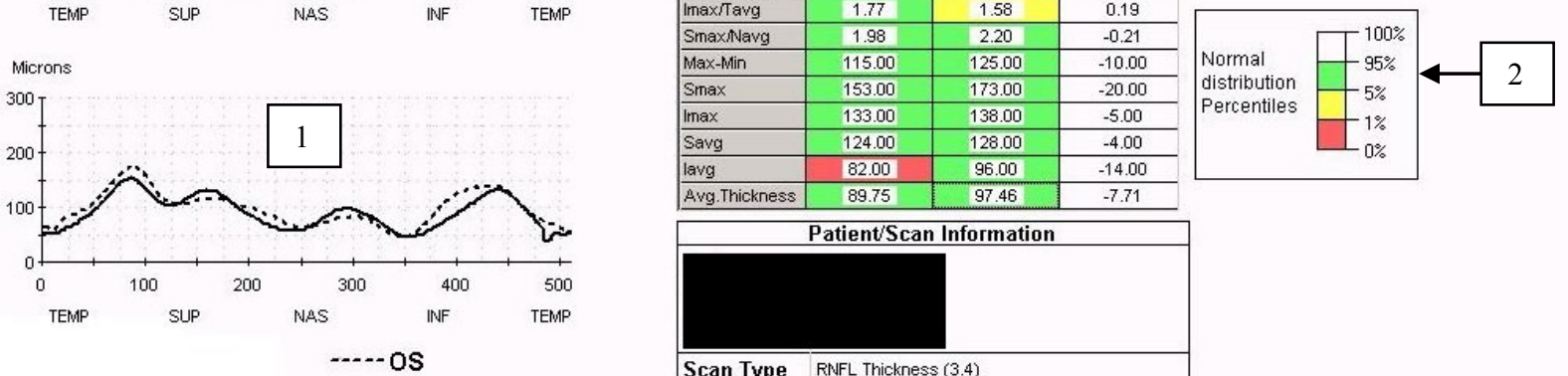

Scan Length $10.87 \mathrm{~mm}$

\section{Abb. 1: Ausdruck einer Nervenfaserschichtdickenmessung mittels Stratus OCT}

Die RNFL-Dickenmessungen werden in TSNIT-Diagrammen (1) graphisch dargestellt und mit einer farbkodierten altersbezogenen normativen Datenbank (2) verglichen. 
Anhang

Tabelle 1: Gruppe I (gesunde Probanden)

\begin{tabular}{|c|c|c|c|c|c|c|c|}
\hline ID & Alter & Refraktion (OD) & Refraktion (OS) & Visus (csc) & Pachymetrie & P-ERG & OCT \\
\hline & & & & & & & \\
\hline $1^{1}$ & 47,4 & I & I & & & $\checkmark$ & I \\
\hline 2 & 55,8 & $+4,5 /-0,25 / 20^{\circ}$ & $+4,75 /-0,75 / 160^{\circ}$ & & & $\checkmark$ & $\checkmark$ \\
\hline $3^{1}$ & 59,6 & & & OD 1,2 OS 1,0 & & $\checkmark$ & 1 \\
\hline 4 & 22,7 & $-2,5 \mathrm{sph}$ & $-1,75 \mathrm{sph}$ & & & $\checkmark$ & $\checkmark$ \\
\hline 5 & 60,2 & $-2,75 /-0,5 / 130^{\circ}$ & $-3,0 /-1,25 / 60^{\circ}$ & OD 0,7 OS 0,5 & & $\checkmark$ & $\checkmark$ \\
\hline 6 & 22,6 & $-1,5 \mathrm{sph}$ & $-1,5 \mathrm{sph}$ & & & $\checkmark$ & $\sqrt{ }$ \\
\hline 7 & 21,8 & S. C. & $-1,0 \mathrm{sph}$ & & & $\checkmark$ & $\sqrt{ }$ \\
\hline 8 & 27,4 & $-0,5 \mathrm{sph}$ & $-0,75 \mathrm{sph}$ & & & $\checkmark$ & $\sqrt{ }$ \\
\hline 9 & 22,4 & S. C. & S. C. & & & $\checkmark$ & $\sqrt{ }$ \\
\hline 10 & 23,6 & $-2,75 \mathrm{sph}$ & $-2,75 \mathrm{sph}$ & & & $\checkmark$ & $\sqrt{ }$ \\
\hline 11 & 23,8 & $-2,75 /-1,25 / 180^{\circ}$ & $-3,5 /-0,75 / 180^{\circ}$ & & & $\checkmark$ & $\checkmark$ \\
\hline 12 & 22,0 & S. C. & S. C. & & & $\checkmark$ & $\checkmark$ \\
\hline 13 & 23,0 & S. C. & $\begin{array}{l}\text { S. C. } \\
\end{array}$ & & & $\checkmark$ & $\checkmark$ \\
\hline 14 & 59,9 & $+1,5 /-6,75 / 15^{\circ}$ & $+0,5 /-0,75 / 88^{\circ}$ add $+0,75$ & bds. 1,0 & & $\checkmark$ & $\checkmark$ \\
\hline 15 & 34,6 & S. C. & $\begin{array}{l}\text { S. C. } \\
\end{array}$ & bds. 1,25 & & $\checkmark$ & $\checkmark$ \\
\hline 16 & 22,7 & $-1,75 \mathrm{sph}$ & $-1,75 \mathrm{sph}$ & & & $\checkmark$ & $\checkmark$ \\
\hline 17 & 58,5 & S. C. & s. C. & & & $\checkmark$ & $\checkmark$ \\
\hline $18^{2}$ & 21,9 & $-6,75 \mathrm{sph}$ & $-7,25 \mathrm{sph}$ & & & $\checkmark$ & $\sqrt{ } \star \Delta$ \\
\hline 19 & 22,5 & S. C. & S. C. & & & $\sqrt{ } \star \star$ & $\checkmark$ \\
\hline $20^{2}$ & 23,6 & s. c. & s. c. & & & $\checkmark$ & $\checkmark$ \\
\hline 21 & 22,0 & $-3,25 \mathrm{sph}$ & $-3,25 \mathrm{sph}$ & & & $\checkmark$ & $\checkmark$ \\
\hline 22 & 21,9 & $+0,25 \mathrm{sph}$ & $+0,5 \mathrm{sph}$ & & & $\checkmark$ & $\checkmark$ \\
\hline 23 & 21,9 & S. C. & S. C. & & & $\checkmark$ & $\checkmark$ \\
\hline $24^{2}$ & 22,3 & $-2,25 \mathrm{sph}$ & $-2,25 \mathrm{sph}$ & & & $\checkmark$ & $\checkmark$ \\
\hline 25 & 23,9 & S. C. & S. C. & & OD $630 \mu \mathrm{m}(-3)$ OS $612 \mu \mathrm{m}(-2)$ & $\checkmark$ & $\checkmark$ \\
\hline 26 & 49,2 & $+2,5 /-0,5 / 71^{\circ}$ add $+1,75$ & $+1,0 /-1,0 / 91^{\circ}$ add $+1,5$ & & & $\checkmark$ & $\checkmark$ \\
\hline 27 & 44,7 & $\begin{array}{l}\text { S. C. } \\
\end{array}$ & S. C. & & & $\checkmark$ & $\checkmark$ \\
\hline 28 & 54,1 & $+0,25 /-0,25 / 78^{\circ}$ add $+1,75$ & cyl $-0,25 / 95^{\circ}$ add $+2,0$ & & & $\checkmark$ & $\checkmark$ \\
\hline 29 & 47,8 & $+0,75 /-0,25 / 20^{\circ}$ add $+0,75$ & $+0,5$ add $+0,75$ & & & $\checkmark$ & $\checkmark$ \\
\hline 30 & 27,9 & $\begin{array}{l}\text { S. C. } \\
\text { S. }\end{array}$ & S. C. & bds. 1,25 & & $\checkmark$ & $\checkmark$ \\
\hline
\end{tabular}

- 48 - 
Tabelle 2: Gruppe II (Kataraktpatienten)

a) Gesund

\begin{tabular}{|l|c|c|c|c|c|c|}
\hline ID & Alter & Refraktion (OD) & Refraktion (OS) & Visus (csc) & Pachymetrie \\
\hline & & & & & & \\
\hline 50 & 53,0 & $+0,75 \mathrm{sph}$ & $+1,0 /-0,25 / 150^{\circ}$ & OD 1,2 OS 0,4 & $/$ & \\
\hline 51 & 60,4 & LB add $+2,00$ & LB add $+2,00$ & & $\checkmark$ & $/$ \\
\hline
\end{tabular}

\section{b) Glaukom-erkrankt}

\begin{tabular}{|l|c|c|c|c|c|c|}
\hline ID & Alter & Refraktion (OD) & Refraktion (OS) & Visus (csc) & Pachymetrie \\
\hline & & & & & & \\
\hline 60 & 68,7 & $-0,75 /-1,0 / 145^{\circ} \mathrm{add}+2,25$ & $-3,25 /-0,75 / 170^{\circ}$ add $+2,5$ & OD 0,8 OS 1,0 & OD $498 \mu \mathrm{m}(+1)$ OS $506 \mu \mathrm{m}(+2)$ & $\checkmark$ \\
\hline 61 & 72,4 & $+6,00 \mathrm{sph}$ & $+5,25 \mathrm{sph}$ & & $/$ \\
\hline
\end{tabular}


Anhang

Tabelle 3: Gruppe III (Glaukompatienten)

\begin{tabular}{|c|c|c|c|c|c|c|c|c|}
\hline ID & Alter & Refraktion (OD) & Refraktion (OS) & Visus (csc) & Pachymetrie & P-ERG & OCT & Diagnose \\
\hline 100 & 74,3 & $\csc$ & $\csc$ & OD 0,8 OS 0,9 & OD $574 \mu \mathrm{m}(-1)$ OS $581 \mu \mathrm{m}(-1)$ & $\bar{\checkmark}$ & $\sqrt{\checkmark \star}$ & POWG (ED '07) \\
\hline 101 & 72,6 & $-7,75 /-0,75 / 25^{\circ}$ & $-6,25 /-4,25 / 180^{\circ}$ & OD 0,6 OS 0,5 & OD $537 \mu \mathrm{m}$ OS $521 \mu \mathrm{m}(+1)$ & $\checkmark$ & $\checkmark$ & POWG (ED '06) \\
\hline 102 & 52,7 & $-2,5 \mathrm{sph}$ & $-1,25 /-0,25 / 10^{\circ}$ & bds. 1,0 & OD $539 \mu \mathrm{m}$ OS $537 \mu \mathrm{m}$ & $\overline{\sqrt{ } \star}$ & $\bar{\checkmark}$ & POWG (ED '06) \\
\hline 103 & 73,5 & S. C. & S. C. & OD 1,0p OS 0,5 & OD $608 \mu \mathrm{m}(-2)$ OS $592 \mu \mathrm{m}(-1)$ & $\checkmark$ & $\sqrt{ } \star$ & POWG (ED '92) \\
\hline 104 & 72,7 & $+3,0 \mathrm{sph}$ & $+4,0 /-0,75 / 100^{\circ}$ & OD 1,0p OS 0,8 & OD $586 \mu \mathrm{m}(-1)$ OS $587 \mu \mathrm{m}(-1)$ & $\sqrt{ }$ & $\checkmark$ & POWG (ED '05) \\
\hline 105 & 68,7 & $-0,75 /-1,0 / 145^{\circ}$ add $+2,25$ & $-3,25 /-0,75 / 170^{\circ}$ add $+2,5$ & OD 0,8 OS 1,0 & OD $498 \mu \mathrm{m}(+1)$ OS $506 \mu \mathrm{m}(+2)$ & $\checkmark$ & $\bar{\checkmark}$ & POWG (ED '02) \\
\hline 106 & 65,3 & $+1,25 /-0,5 / 90^{\circ}$ & $+1,0 \mathrm{sph}$ & OD 0,8 OS $1,0 p$ & OD $536 \mu \mathrm{m}$ OS $535 \mu \mathrm{m}$ & $\checkmark$ & $\sqrt{ } \star$ & POWG (ED '92) \\
\hline 107 & 81,6 & $+1,25 /-1,25 / 95^{\circ}$ & $+3,0 /-2,5 / 88$ & OD 0,7 OS 0,8 & OD $571 \mu \mathrm{m}$ OS $554 \mu \mathrm{m}$ & $\checkmark$ & $\checkmark$ & POWG (ED '03) \\
\hline 108 & 57,0 & $+0,5 /-1,5 / 90^{\circ}$ & $+0,75 /-1,75 / 90^{\circ}$ & OD $0,8 p$ OS 1,0 & OD $572 \mu \mathrm{m}$ OS $580 \mu \mathrm{m}(-1)$ & $\checkmark$ & $\sqrt{ } \star$ & POWG (ED'00) \\
\hline 109 & 75,6 & $+2,0 /-0,5 / 84^{\circ}$ & $+2,5 /-0,75 / 108^{\circ}$ & OD 0,7 OS 0,5 & OD $570 \mu \mathrm{m}(-1)$ OS $553 \mu \mathrm{m}$ & $\checkmark$ & $\sqrt{ } \star$ & POWG (ED '06) \\
\hline 110 & 69,4 & $+1,25 /-0,25 / 75^{\circ}$ & $+1,25 /-0,25 / 85^{\circ}$ & bds. 0,7 & OD $620 \mu \mathrm{m}(-2)$ OS $630 \mu \mathrm{m}(-3)$ & $\checkmark$ & $\checkmark$ & POWG (ED '05) \\
\hline 111 & 70,2 & $-2,75 /-0,25 / 86^{\circ}$ & $-0,25 /-0,25 / 68^{\circ}$ & bds. 0,8 & OD $559 \mu \mathrm{m}$ OS $562 \mu \mathrm{m}$ & $\checkmark$ & $\sqrt{ } \star$ & POWG \\
\hline 112 & 83,7 & $+1,75 /-0,25 / 11^{\circ}$ add $+3,0$ & $+1,5 /-0,25 / 68^{\circ}$ add $+3,0$ & OD $0,8 p$ OS 0,6 & OD $531 \mu \mathrm{m}$ OS $512 \mu \mathrm{m}(+1)$ & $\checkmark$ & $\sqrt{ } \star$ & POWG (ED '05) \\
\hline 113 & 55,4 & $+1,5 /-0,25 / 78^{\circ}$ & $+1,25 /-0,25 / 79^{\circ}$ & OD 1,0 OS 0,8 & OD $609 \mu \mathrm{m}(-2)$ OS $602 \mu \mathrm{m}(-2)$ & $\overline{\sqrt{ } \star}$ & $\overline{\sqrt{ } \star}$ & NDG \\
\hline 114 & 49,0 & $-2,75 /-1,5 / 86^{\circ}$ & $-3,5 /-1,5 / 60^{\circ}$ & bds. 1,0 & OD $541 \mu \mathrm{m}$ OS $538 \mu \mathrm{m}$ & $\sqrt{ } \star$ & $\sqrt{ } \star$ & POWG (ED '09) \\
\hline 115 & 48,9 & $-1,0 \mathrm{sph}$ & $-1,25 \mathrm{sph}$ & bds. 1,0 & OD $579 \mu \mathrm{m}$ OS $586 \mu \mathrm{m}$ & $\sqrt{ } \star$ & $\sqrt{ } \star$ & POWG (ED '07) \\
\hline 116 & 69,4 & $+1,75 /-0,5 / 85^{\circ}$ & $+0,5 /-0,25 / 70^{\circ}$ & OD 1,0 OS 0,9 & OD $521(+1)$ OS $486(+2)$ & $\checkmark$ & $\checkmark$ & POWG (ED '07) \\
\hline 117 & 48,6 & S. C. & S. C. & OD 1,0p OS0,9 & 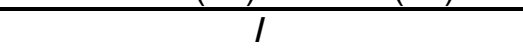 & $\checkmark$ & $\checkmark$ & POWG (ED '06) \\
\hline 118 & 56,8 & $+0,25 /-0,25 / 100^{\circ}$ & $+0,25 /-0,25 / 130^{\circ}$ & bds. $1,0 p$ & OD $575 \mu \mathrm{m}$ OS $578 \mu \mathrm{m}$ & $\checkmark$ & $\sqrt{ } \star$ & POWG (ED '98) \\
\hline 119 & 73,1 & $+1,5 /-0,25 / 78^{\circ}$ & $+1,75 /-0,5 / 91^{\circ}$ & bds. 1,0 & OD $613 \mu \mathrm{m}(-2)$ OS $617 \mu \mathrm{m}(-2)$ & $\checkmark$ & $\sqrt{ } \star$ & NDG (ED '02) \\
\hline
\end{tabular}


Anhang

Fortsetzung von Tabelle 3: Gruppe III (Glaukompatienten)

\begin{tabular}{|c|c|c|c|c|c|c|c|c|}
\hline ID & GF & MD-D & PSD-D & MD-S & PSD-S & IOD-D & IOD-S & Besonderheiten \\
\hline & & {$[\mathrm{dB}]$} & {$[\mathrm{dB}]$} & {$[\mathrm{dB}]$} & {$[\mathrm{dB}]$} & {$[\mathrm{mmHg}]$} & {$[\mathrm{mmHg}]$} & \\
\hline 100 & $\sqrt{ } \star$ & $-3,03$ & 6,8 & $-4,46$ & 9,83 & 14 & 14 & Cat. incip.; CDR OD 0,7 OS 0,6 \\
\hline 101 & $\sqrt{ } \star$ & $-3,99$ & 3,45 & $-2,65$ & 1,87 & 19 & 15 & Cat. incip.; Pap. mit temp. Randsaumatrophie \\
\hline 102 & $\checkmark$ & 0,28 & 2,14 & 0,11 & 2,2 & 16 & 13 & CDR OD 0,7 (bis Lamina cribrosa sichtbar) OS 0,6 \\
\hline 103 & $\sqrt{ } \star \sqrt{4}$ & $-4,45$ & 4 & $-5,38$ & 7,05 & 13 & 14 & Cat. incip.; CDR bds. 0,8 \\
\hline 104 & $\checkmark$ & 1,24 & 2,05 & 0,01 & 2 & 19 & 20 & Cat. incip.; ISNT nicht erfüllt, CDR 0,5 \\
\hline 105 & $\checkmark$ & $-2,28$ & 1,48 & $-0,37$ & 2,11 & 15 & 15 & OD pseudophak, OS Cat. incip.; CDR 0,8 \\
\hline 106 & $\sqrt{ } \sqrt{2 \star}$ & $-4,14$ & 7,28 & $-10,4$ & 13,62 & 16 & 16 & Cat. incip.; Pap. bds. glaukomatös exkaviert, 6h randständig \\
\hline 107 & $\sqrt{ } \star$ & $-1,42$ & 1,67 & $-5,36$ & 12,29 & 25 & 19 & Cat. incip.; CDR OD 0,8 OS 0,6 \\
\hline 108 & $\sqrt{ } \star$ & $-9,12$ & 9,15 & $-0,94$ & 1,74 & 16 & 13 & Cat. incip.; CDR OD 0,8 OS 0,6 \\
\hline 109 & $\sqrt{ } \star$ & $-2,36$ & 3,39 & $-5,51$ & 6,29 & 16 & 15 & Cat. inc.; CDR 0,9 \\
\hline 110 & $\checkmark$ & $-0,78$ & 2,11 & $-0,94$ & 2,22 & 18 & 19 & Cat. incip.; bds. Lam. cribrosa sichtbar; CDR 0,7 \\
\hline 111 & $\sqrt{ } \star$ & $-1,77$ & 1,86 & $-2,41$ & 3,67 & 25 & 24 & Cat. incip.; Fam.-A. pos. \\
\hline 112 & $\sqrt{ } \star$ & $-0,34$ & 1,84 & $-4,82$ & 6,94 & 13 & 16 & CDR OD 0,7 OS 0,8 \\
\hline 113 & $\sqrt{ } \star$ & $-5,14$ & 9,49 & $-13,4$ & 14,05 & 11 & 12 & Cat. incip.; CDR: OD 0,6 OS 0,7 \\
\hline 114 & $\sqrt{ } \star \Delta$ & $-2,11$ & 2,16 & $-1,91$ & 2,21 & 15 & 14 & Cat. incip.; ISNT nicht erfüllt, CDR 0,7-0,8 \\
\hline 115 & $\checkmark$ & 1,99 & 1,7 & 1,57 & 1,64 & 17 & 18 & Pap. zentral tief exkaviert, CDR bds. 0,7 \\
\hline 116 & $\sqrt{ } \star$ & $-3,21$ & 3,41 & $-6,01$ & 5,5 & 20 & 20 & Cat. incip.; CDR OD 0,8 OS 0,9 temp. randständig exkaviert \\
\hline 117 & $\checkmark$ & 0,94 & 1,79 & 1,16 & 1,44 & 14 & 16 & Fam.-A. pos.; CDR OD 0,6 OS 0,6-0,7 \\
\hline 118 & $\sqrt{ } \star \sqrt{2}$ & $-4,59$ & 8,64 & $-3,23$ & 5,73 & 14 & 14 & Opticusatrophie II-III; CDR 0,8 \\
\hline 119 & $\sqrt{ } \star$ & $-5,32$ & 6,14 & $-6,5$ & 7,01 & 15 & 14 & CDR 0,8 \\
\hline
\end{tabular}


Anhang

Tabelle 4: Gruppe IV (Verdachtspatienten)

a) Eher Glaukom-manifest

\begin{tabular}{|c|c|c|c|c|c|c|c|c|}
\hline ID & Alter & Refraktion (OD) & Refraktion (OS) & Visus (csc) & Pachymetrie & P-ERG & OCT & Th. It. Brief \\
\hline & & & & & & & & \\
\hline 200 & 70,7 & $+2,75 /-0,25 / 160^{\circ}$ & $+3,75 /-0,75 / 171^{\circ}$ & bds. 0,9 & OD $563 \mu \mathrm{m}$ OS $527 \mu \mathrm{m}$ & $\checkmark$ & $\checkmark$ & ja \\
\hline 201 & 23,9 & $-6,25 \mathrm{sph}$ & $-5,5 /-0,25 / 180^{\circ}$ & bds. 1,25 & OD $586 \mu \mathrm{m}(-1)$ OS $584 \mu \mathrm{m}(-1)$ & $\checkmark$ & $\checkmark$ & ja \\
\hline 202 & 18,9 & S. C. & S. C. & bds. 0,8 & OD $593 \mu \mathrm{m}(-1)$ OS $587 \mu \mathrm{m}(-1)$ & $\checkmark$ & $\sqrt{\star \star}$ & ja \\
\hline 203 & 25,9 & $-4,0 /-0,5 / 70^{\circ}$ & $-5,5 \mathrm{sph}$ & OD 1,0 OS 1,0 & OD $543 \mu \mathrm{m}$ OS $550 \mu \mathrm{m}$ & $\checkmark$ & $\checkmark$ & ja \\
\hline 204 & 69,4 & $+1,25 /-0,5 / 38$ & $+1,75 /-1,25 / 67$ & bds. 1,0 & OD $542 \mu \mathrm{m}$ OS $507 \mu \mathrm{m}(+1)$ & $\checkmark$ & $\sqrt{\checkmark \star}$ & ja \\
\hline 205 & 32,8 & $-1,5 /-0,5 / 163^{\circ}$ & $-0,75 /-1,0 / 130^{\circ}$ & bds. 1,2 & bds. $+5 \mathrm{mmHg}$ & $\checkmark$ & $\checkmark$ & ja \\
\hline 206 & 37,4 & $-2,25 /-1,0 / 135^{\circ}$ & $-3,5 /-0,8 / 59^{\circ}$ & bds. 1,25 & OD $553 \mu \mathrm{m}$ OS $576 \mu \mathrm{m}$ & $\sqrt{ } \star \star$ & $\checkmark \star$ & ja \\
\hline 207 & 67,7 & $\left({ }^{\prime}-0,5 /-0,5 / 108^{\circ}\right)$ & $('-1,5)$ & OD 0,8 OS 0,7 & OD $557 \mu \mathrm{m}$ OS $547 \mu \mathrm{m}$ & $\checkmark$ & $\sqrt{\checkmark \star}$ & ja \\
\hline 208 & 18,0 & $-4,0 /-0,75 / 14^{\circ}$ & $-4,25 /-1,5 / 177^{\circ}$ & bds. 1,0 & OD $512 \mu \mathrm{m}(+1)$ OS $517 \mu \mathrm{m}(+1)$ & $\checkmark$ & $\checkmark$ & OD Travatan z.N. \\
\hline 209 & 58,5 & $+1,25 /-0,25 / 152^{\circ}$ add $+2,25$ & $+0,5 /-0,25 / 157^{\circ}$ add $+2,5$ & OD 1,0 OS 1,0 & OD $535 \mu \mathrm{m}$ OS $507 \mu \mathrm{m}(+1)$ & $\checkmark$ & $\checkmark$ & ja \\
\hline 210 & 45,2 & $+0,75 /-0,5 / 110^{\circ}$ & $+0,25 \mathrm{sph}$ & OD 0,8 OS 1,0 & OD $578 \mu \mathrm{m}(-1)$ OS $582 \mu \mathrm{m}(-1)$ & $\checkmark$ & $\checkmark$ & ja \\
\hline 211 & 31,6 & $+0,25 /-0,5 / 120^{\circ}$ & $+0,25 /-0,75 / 65^{\circ}$ & bds. 1,0 & OD $558 \mu \mathrm{m}$ OS $562 \mu \mathrm{m}(-1)$ & $\sqrt{ }$ & $\sqrt{ } \star$ & ja \\
\hline 212 & 49,4 & $+1,5 /-0,25 / 132^{\circ}$ & $+1,25 /-0,25 / 132^{\circ}$ & OD 0,8 OS 1,0 & OD $634 \mu \mathrm{m}(-3)$ OS $639 \mu \mathrm{m}(-3)$ & $\bar{v}$ & $\checkmark$ & ja \\
\hline
\end{tabular}

b) Eher kein Glaukom

\begin{tabular}{|c|c|c|c|c|c|c|c|c|}
\hline ID & Alter & Refraktion (OD) & Refraktion (OS) & Visus (csc) & Pachymetrie & P-ERG & OCT & Th. It. Brief \\
\hline 213 & 29,1 & orthokeratologe CL & orthokeratologe CL & bds. 1,2 & OD $558 \mu \mathrm{m}$ OS $549 \mu \mathrm{m}$ & $\checkmark \star$ & $\checkmark$ & nein \\
\hline 214 & 66,8 & $+3,75 /-0,75 / 75^{\circ}$ & $+2,75 \mathrm{sph}$ & bds. 1,0 & OD $570 \mu \mathrm{m}(-1)$ OS $582 \mu \mathrm{m}(-1)$ & $\checkmark$ & $\checkmark$ & nein \\
\hline 215 & 69,6 & $+1,75 /-0,5 / 180^{\circ}$ & $+2,0 /-0,25 / 120^{\circ}$ & bds. 0,7 & OD $608 \mu \mathrm{m}(-2)$ OS $612 \mu \mathrm{m}(-2)$ & $\checkmark$ & $\checkmark$ & nein \\
\hline 216 & 45,9 & $-4,5 /-0,5 / 33^{\circ}$ & $-3,25 /-1,0 / 163^{\circ}$ & OD 1,2 OS 1,0 & bds. $561 \mu \mathrm{m}$ & $\checkmark$ & $\checkmark$ & nein \\
\hline 217 & 21,8 & S. C. & S. C. & bds. 1,0 & OD $525 \mu \mathrm{m}(+1)$ OS $532 \mu \mathrm{m}$ & $\checkmark$ & $\checkmark$ & nein \\
\hline 218 & 37,8 & S. C. & S. C. & bds. 1,0 & OD $509 \mu \mathrm{m}(+1)$ OS $507 \mu \mathrm{m}(1)$ & $\checkmark$ & $\checkmark$ & nein \\
\hline 219 & 40,3 & $-4,75 /-0,75 / 15^{\circ}$ & $-4,75 \mathrm{sph}$ & bds. 1,0 & OD $564 \mu \mathrm{m}(-1)$ OS $571 \mu \mathrm{m}(-1)$ & $\checkmark$ & $\checkmark$ & nein \\
\hline
\end{tabular}


Anhang

Fortsetzung von Tabelle 4: Gruppe IV (Verdachtspatienten)

a) Eher Glaukom-manifest

\begin{tabular}{|l|c|c|c|c|c|c|c|c|}
\hline ID & GF & MD-D & PSD-D & MD-S & PSD-S & IOD-D & IOD-S & Besonderheiten \\
\hline & & {$[\mathrm{dB}]$} & {$[\mathrm{dB}]$} & {$[\mathrm{dB}]$} & {$[\mathrm{dB}]$} & {$[\mathrm{mmHg}]$} & {$[\mathrm{mmH}]$} & \\
\hline 200 & $\checkmark \star$ & $-0,5$ & 2,59 & $-4,06$ & 6,42 & 11 & 9 & Cat. incip. OD>OS; OS Bjerrum-Skotom; CDR OD 0,6 OS 0,4 \\
\hline 201 & $\checkmark$ & 0,4 & 1,41 & 0,41 & 1,39 & 14 & 14 & bds. antiglaukomatöse Th.; farbig; Gonio bds. stark pigment. Synech.; CDR bds. 0,6-0,7 \\
\hline 202 & $\checkmark \star$ & $-4,8$ & 10,57 & $-0,11$ & 1,45 & 22 & 21 & seit 5 Wo. unklare GF-Defekte \\
\hline 203 & $\checkmark$ & $-0,33$ & 1,42 & $-0,04$ & 1,54 & 19 & 19 & bds. antiglaukomatöse Th.; Fam.-A. pos.; Pap. zentral tief exkaviert; CDR OD 0,5 OS 0,6 \\
\hline 204 & $\checkmark$ & 1,23 & 1,91 & $-0,44$ & 2,12 & 11 & 12 & bds. antiglaukomatöse Th.; Cat. incip.; CDR bds. 0,7 \\
\hline 205 & $\checkmark$ & 1,04 & 1,52 & 0,28 & 1,7 & 15 & 16 & bds. antiglaukomatöse Th.; seit 18. Lj. IOD bis 30 mmHg; CDR bds. 0,3 \\
\hline 206 & $\checkmark \star$ & $-1,52$ & 3,81 & $-5,43$ & 5,81 & 11 & 11 & bds. antiglaukomatöse Th.; bds. bg. GF-Ausfälle OS $>$ OD; Pap. bds. flach; CDR 0,6 \\
\hline 207 & $\checkmark \star$ & 0,61 & 1,73 & -4 & 6,75 & 16 & 15 & OS bogenförmiges Skotom; CDR OD 0,5 OS 0,6 \\
\hline 208 & $\checkmark$ & $-2,69$ & 1,63 & $-3,54$ & 2,3 & 13 & 10 & bds. zirk. Goniosynechien; CDR OD 0,8 OS 0,6 \\
\hline 209 & $\checkmark$ & 0,25 & 1,23 & 0,22 & 1,24 & 11 & 14 & OD inf. Randsaum etwas ausgedünnt; ISNT nicht erfüllt; CDR 0,6 \\
\hline 210 & $\checkmark \star$ & $-3,39$ & 4,04 & $-3,67$ & 3,75 & 16 & 17 & CDR OD 0,5 OS 0,6-0,7 \\
\hline 211 & $\checkmark$ & $-2,01$ & 2,21 & $-2,5$ & 2,16 & 20 & 18 & bds. antiglaukomatöse Th.; häufige IOD-Erhöhungen \\
\hline 212 & $\checkmark$ & 0,73 & 1,07 & 0 & 1,67 & 16 & 15 & CDR OD 0,7 OS 0,6 \\
\hline
\end{tabular}

\section{b) Eher kein Glaukom}

\begin{tabular}{|l|c|c|c|c|c|c|c|c|}
\hline ID & GF & MD-D & PSD-D & MD-S & PSD-S & IOD-D & IOD-S & Besonderheiten \\
\hline & & {$[\mathrm{dB}]$} & {$[\mathrm{dB}]$} & {$[\mathrm{dB}]$} & {$[\mathrm{dB}]$} & {$[\mathrm{mmHg}]$} & {$[\mathrm{mmHg}]$} & \\
\hline 213 & $\checkmark$ & 0,7 & 1,63 & 0,6 & 1,45 & 13 & 13 & OS ISNT nicht erfüllt; CDR OD 0,8-0,9 OS 0,7 \\
\hline 214 & $\checkmark$ & $-0,61$ & 2,39 & $-0,22$ & 2,38 & 14 & 13 & pos. Fam.-A.; Cat. incip.; CDR OD 0,6 OS 0,5 \\
\hline 215 & $\checkmark$ & $-1,96$ & 2,64 & $-0,23$ & 1,58 & 15 & 13 & Cat. incip.; CDR OD 0,6 OS 0,7 \\
\hline 216 & $\checkmark$ & 0,89 & 1,46 & 0,21 & 1,52 & 13 & 13 & CDR OD 0,5 OS 0,6 \\
\hline 217 & $\checkmark \star$ & $-3,44$ & 3,54 & $-5,11$ & 4,52 & 13 & 14 & Makropapille, CDR OD 0,6 OS 0,7 \\
\hline 218 & $\checkmark$ & $-0,66$ & 1,54 & $-1,34$ & 1,5 & 14 & 14 & CRD OD 0,4 OS 0,6 \\
\hline 219 & $\checkmark \star$ & $-1,07$ & 5,05 & $-0,46$ & 1,99 & 13 & 14 & Pap. blass, exkav., temp. Atrophie, CDR 0,5 \\
\hline
\end{tabular}

$$
\begin{array}{l|l|l}
<2 \% & <1 \% & <0,5 \%
\end{array}
$$


Anhang

Tabelle 6: P-ERG- und OCT-Ergebnisse der Gruppe I (gesunde Probanden)

\begin{tabular}{|c|c|c|c|c|c|c|c|c|c|c|c|}
\hline ID & Alter & P-ERG $[\mu \mathrm{V}]$ & B-P-ERG $[\mu \mathrm{V}]$ & B-weiß [\%] & OCT & $\varnothing \mathrm{RNFL}[\mu \mathrm{m}]$ & P-ERG [ $\mu \mathrm{V}]$ & B-P-ERG $[\mu \mathrm{V}]$ & B-weiß [\%] & OCT & $\varnothing \mathrm{RNFL}[\mu \mathrm{m}]$ \\
\hline & & \multicolumn{5}{|c|}{ 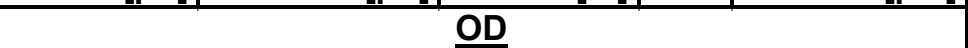 } & \multicolumn{5}{|c|}{ OS } \\
\hline 1 & 47,4 & 3,05 & 0,43 & 14,1 & 1 & 1 & 3,05 & 0,61 & 20,0 & I & 1 \\
\hline 2 & 55,8 & 3,66 & 0,61 & 16,7 & 12 & 110,20 & 3,48 & 1,22 & 35,1 & 12 & 99,39 \\
\hline 3 & 59,6 & 3,66 & 0,00 & 0,0 & 1 & 1 & 3,66 & 0,00 & 0,0 & 1 & 1 \\
\hline 4 & 22,7 & 3,66 & 2,13 & 58,2 & 10 & 94,12 & 3,05 & 2,44 & 80,0 & 12 & 92,40 \\
\hline 5 & 60,2 & 3,66 & 0,00 & 0,0 & 12 & 95,66 & 1,83 & 0,00 & 0,0 & 12 & 83,71 \\
\hline 6 & 22,6 & 4,88 & 2,44 & 50,0 & 11 & 98,33 & 5,49 & 3,35 & 61,0 & 9 & 93,70 \\
\hline 7 & 21,8 & 6,70 & 3,96 & 59,1 & 12 & 112,65 & 7,32 & 3,66 & 50,0 & 12 & 109,33 \\
\hline 8 & 27,4 & 6,40 & 2,87 & 44,8 & 11 & 98,58 & 6,52 & 3,05 & 46,8 & 12 & 98,06 \\
\hline 9 & 22,4 & 5,91 & 2,87 & 48,6 & 12 & 100,08 & 5,79 & 2,74 & 47,3 & 12 & 108,14 \\
\hline 10 & 23,6 & 4,27 & 1,83 & 42,9 & 12 & 94,49 & 4,88 & 3,05 & 62,5 & 12 & 92,15 \\
\hline 11 & 23,8 & 5,61 & 3,96 & 70,6 & 11 & 103,46 & 5,49 & 3,35 & 61,0 & 10 & 92,44 \\
\hline 12 & 22,0 & 2,87 & 2,26 & 78,7 & 12 & 105,94 & 1,83 & 1,83 & 100,0 & 12 & 104,54 \\
\hline 13 & 23,0 & 4,70 & 3,66 & 77,9 & 12 & 101,89 & 4,88 & 4,24 & 86,9 & 12 & 92,38 \\
\hline 14 & 59,9 & 3,66 & 1,22 & 33,3 & 12 & 98,38 & 3,35 & 1,65 & 49,3 & 11 & 85,40 \\
\hline 15 & 34,6 & 7,32 & 4,88 & 66,7 & 12 & 112,54 & 7,32 & 3,05 & 41,7 & 12 & 114,35 \\
\hline 16 & 22,7 & 9,15 & 5,49 & 60,0 & 12 & 89,09 & 5,49 & 3,05 & 55,6 & 12 & 91,03 \\
\hline 17 & 58,5 & 4,88 & 1,22 & 25,0 & 12 & 92,11 & 6,52 & 2,87 & 44,0 & 11 & 87,94 \\
\hline 18 & 21,9 & 4,88 & 2,87 & 58,8 & 6 & 81,82 & 6,40 & 3,05 & 47,7 & 6 & 83,18 \\
\hline 19 & 22,5 & 5,49 & 1,95 & 35,5 & 12 & 110,58 & 6,10 & 3,05 & 50,0 & 12 & 116,67 \\
\hline 20 & 23,6 & 5,49 & 3,48 & 63,4 & 12 & 96,29 & 5,49 & 2,87 & 52,3 & 12 & 91,06 \\
\hline 21 & 22,0 & 9,45 & 5,79 & 61,3 & 12 & 105,20 & 9,76 & 4,57 & 46,8 & 11 & 106,86 \\
\hline 22 & 21,9 & 6,71 & 4,88 & 72,7 & 11 & 90,10 & 6,71 & 4,27 & 63,6 & 12 & 95,34 \\
\hline 23 & 21,9 & 7,93 & 3,66 & 46,2 & 12 & 103,15 & 8,54 & 3,78 & 44,3 & 12 & 109,76 \\
\hline 24 & 22,3 & 6,10 & 3,66 & 60,0 & 12 & 91,70 & 4,88 & 4,75 & 97,3 & 12 & 91,70 \\
\hline 25 & 23,9 & 6,71 & 4,88 & 72,7 & 9 & 84,36 & 6,71 & 3,05 & 45,5 & 10 & 82,58 \\
\hline 26 & 49,2 & 4,39 & 1,65 & 37,6 & 12 & 111,35 & 2,87 & 1,22 & 42,5 & 12 & 112,36 \\
\hline 27 & 44,7 & 6,10 & 2,56 & 42,0 & 12 & 120,97 & 7,32 & 2,44 & 33,3 & 12 & 111,26 \\
\hline 28 & 54,1 & 4,27 & 1,04 & 24,4 & 10 & 98,26 & 2,74 & 0,61 & 22,3 & 11 & 91,99 \\
\hline 29 & 47,8 & 4,57 & 1,83 & 40,0 & 12 & 100,21 & 3,66 & 1,83 & 50,0 & 12 & 95,62 \\
\hline 30 & 27,9 & 4,88 & 3,05 & 62,5 & 12 & 104,84 & 6,10 & 2,26 & 37,0 & 11 & 107,34 \\
\hline$\overline{M W}$ & 33,7 & 5,37 & 2,70 & 47,5 & 11,4 & 100,23 & 5,24 & 2,60 & 49,1 & 11,4 & 97,88 \\
\hline
\end{tabular}

- 54 - 
Tabelle 7: Alterskorrelierte Blau-zu-Weiß-Muster-ERG-Normwerte (90\%-Referenzbereich)

\begin{tabular}{|llll|}
\hline Alter [Jahre] & Untergrenze & Mittelwert & Obergrenze \\
\hline
\end{tabular}

\begin{tabular}{|llll|}
\hline 20 & 38,38 & 62,11 & 85,84 \\
21 & 37,34 & 61,05 & 84,76 \\
22 & 36,31 & 59,99 & 83,67 \\
23 & 35,27 & 58,93 & 82,60 \\
24 & 34,23 & 57,87 & 81,52 \\
\hline
\end{tabular}

\begin{tabular}{|llll|}
\hline 25 & 33,19 & 56,82 & 80,44 \\
26 & 32,14 & 55,76 & 79,37 \\
27 & 31,10 & 54,70 & 78,30 \\
28 & 30,05 & 53,64 & 77,23 \\
29 & 29,00 & 52,58 & 76,16 \\
\hline
\end{tabular}

\begin{tabular}{|llll|}
\hline 30 & 27,95 & 51,52 & 75,10 \\
31 & 26,90 & 50,46 & 74,03 \\
32 & 25,84 & 49,41 & 72,97 \\
33 & 24,78 & 48,35 & 71,91 \\
34 & 23,73 & 47,29 & 70,85 \\
\hline
\end{tabular}

\begin{tabular}{|llll|}
\hline 35 & 22,67 & 46,23 & 69,79 \\
36 & 21,61 & 45,17 & 68,74 \\
37 & 20,54 & 44,11 & 67,68 \\
38 & 19,48 & 43,05 & 66,63 \\
39 & 18,41 & 42,00 & 65,58 \\
\hline
\end{tabular}

\begin{tabular}{|llll|}
\hline 40 & 17,34 & 40,94 & 64,53 \\
41 & 16,27 & 39,88 & 63,49 \\
42 & 15,20 & 38,82 & 62,44 \\
43 & 14,12 & 37,76 & 61,40 \\
44 & 13,05 & 36,70 & 60,36 \\
\hline
\end{tabular}

\begin{tabular}{|llll|}
\hline 45 & 11,97 & 35,65 & 59,32 \\
46 & 10,89 & 34,59 & 58,28 \\
47 & 9,81 & 33,53 & 57,25 \\
48 & 8,73 & 32,47 & 56,21 \\
49 & 7,64 & 31,41 & 55,18 \\
\hline
\end{tabular}

\begin{tabular}{|llll|}
\hline 50 & 6,56 & 30,35 & 54,15 \\
51 & 5,47 & 29,29 & 53,12 \\
52 & 4,38 & 28,24 & 52,09 \\
53 & 3,29 & 27,18 & 51,07 \\
54 & 2,19 & 26,12 & 50,04 \\
\hline
\end{tabular}

\begin{tabular}{|llll|}
\hline 55 & 1,10 & 25,06 & 49,02 \\
56 & 0,00 & 24,00 & 48,00 \\
57 & 0,00 & 22,94 & 46,98 \\
58 & 0,00 & 21,89 & 45,97 \\
59 & 0,00 & 20,83 & 44,95 \\
60 & 0,00 & 19,77 & 43,94 \\
\hline
\end{tabular}


Tabelle 8: P-ERG- und OCT-Ergebnisse der Gruppe II (Kataraktpatienten)

a) Gesund (bds. pseudophak)

\begin{tabular}{|c|c|c|c|c|c|c|c|c|c|c|c|}
\hline ID & Alter & P-ERG $[\mu V]$ & B-P-ERG $[\mu \mathrm{V}]$ & B-weiß [\%] & OCT & $\varnothing \mathrm{RNFL}[\mu \mathrm{m}]$ & P-ERG $[\mu \mathrm{V}]$ & B-P-ERG $[\mu \mathrm{V}]$ & B-weiß [\%] & OCT & $\varnothing \mathrm{RNFL}[\mu \mathrm{m}]$ \\
\hline & & \multicolumn{5}{|c|}{ OD } & \multicolumn{5}{|c|}{ OS } \\
\hline 50 & 53,0 & 3,05 & 1,52 & 49,8 & 1 & I & 2,74 & 1,83 & 66,8 & 1 & 1 \\
\hline 51 & 60,4 & 5,18 & 1,22 & 23,6 & I & I & 6,71 & 2,44 & 36,4 & 1 & I \\
\hline MW & 56,7 & 4,12 & 1,37 & 36,7 & 1 & 1 & 4,73 & 2,14 & 51,6 & 1 & 1 \\
\hline SD & 5,2 & 1,51 & 0,21 & 18,6 & 1 & I & 2,81 & 0,43 & 21,5 & 1 & 1 \\
\hline Max & 60,4 & 5,18 & 1,52 & 49,8 & 1 & 1 & 6,71 & 2,44 & 66,8 & 1 & 1 \\
\hline Min & 53,0 & 3,05 & 1,22 & 23,6 & 1 & 1 & 2,74 & 1,83 & 36,4 & 1 & 1 \\
\hline SW & 7,4 & 2,13 & 0,30 & 26,3 & I & I & 3,97 & 0,61 & 30,4 & I & I \\
\hline
\end{tabular}

b) Glaukom-erkrankt (beide OD pseudophak)

\begin{tabular}{|c|c|c|c|c|c|c|c|c|c|c|c|}
\hline ID & Alter & P-ERG $[\mu V]$ & B-P-ERG $[\mu \mathrm{V}]$ & B-weiß [\%] & OCT & $\varnothing \mathrm{RNFL}[\mu \mathrm{m}]$ & P-ERG $[\mu V]$ & B-P-ERG $[\mu \mathrm{V}]$ & B-weiß [\%] & OCT & Ø RNFL [ $\mu \mathrm{m}]$ \\
\hline & & \multicolumn{5}{|c|}{ OD } & \multicolumn{5}{|c|}{ OS } \\
\hline 60 & 68,7 & 2,13 & 0,00 & 0,0 & 6 & 67 & 3,66 & 1,04 & 28,4 & 12 & 83 \\
\hline 61 & 72,0 & 4,88 & 0,00 & 0,0 & l & I & 1,65 & 0,00 & 0,0 & I & l \\
\hline
\end{tabular}

\begin{tabular}{|l|c|c|c|c|c|c|c|c|c|c|c|}
\hline MW & 70,4 & 3,51 & 0,00 & 0,0 & 6,0 & 67,0 & 2,66 & 0,52 & 14,2 & 12,0 & 83,0 \\
\hline SD & 2,3 & 1,94 & 0,00 & 0,0 & $/$ & $/$ & 1,42 & 0,74 & 20,1 & $/$ & $/$ \\
\hline Max & 72,0 & 4,88 & 0,00 & 0,0 & 6,0 & 67,0 & 3,66 & 1,04 & 28,4 & 12,0 & 83,0 \\
\hline Min & 68,7 & 2,13 & 0,00 & 0,0 & 6,0 & 67,0 & 1,65 & 0,00 & 0,0 & 12,0 & 83,0 \\
\hline SW & 3,3 & 2,75 & 0,00 & 0,0 & 0,0 & 0,0 & 2,01 & 1,04 & 28,4 & 0,0 & 0,0 \\
\hline
\end{tabular}


Anhang

Tabelle 9: P-ERG- und OCT-Ergebnisse der Gruppe III (Glaukompatienten)

\begin{tabular}{|c|c|c|c|c|c|c|c|c|c|c|c|}
\hline ID & Alter & P-ERG $[\mu \mathrm{V}]$ & B-P-ERG $[\mu \mathrm{V}]$ & B-weiß [\%] & OCT & $\varnothing$ RNFL $[\mu \mathrm{m}]$ & P-ERG $[\mu V]$ & B-P-ERG $[\mu \mathrm{V}]$ & B-weiß [\%] & OCT & $\varnothing \mathrm{RNFL}[\mu \mathrm{m}]$ \\
\hline & & \multicolumn{5}{|c|}{ OD } & \multicolumn{5}{|c|}{ OS } \\
\hline 100 & 74,3 & 0,00 & 0,00 & 0,0 & 10 & 68,89 & 3,17 & 0,00 & 0,0 & 12 & 78,95 \\
\hline 101 & 72,6 & 0,00 & 0,00 & 0,0 & 11 & 89,59 & 1,52 & 0,00 & 0,0 & 9 & 86,98 \\
\hline 102 & 52,7 & 0,00 & 0,00 & 0,0 & 11 & 89,43 & 1,52 & 0,00 & 0,0 & 10 & 96,19 \\
\hline 103 & 73,5 & 2,13 & 0,00 & 0,0 & 10 & 74,04 & 2,13 & 0,00 & 0,0 & 9 & 67,02 \\
\hline 104 & 72,7 & 0,61 & 0,00 & 0,0 & 11 & 94,04 & 1,83 & 0,00 & 0,0 & 12 & 90,50 \\
\hline 105 & 68,7 & \multicolumn{5}{|c|}{ Pseudophakie } & 3,66 & 1,04 & 28,4 & 12 & 83,00 \\
\hline 106 & 65,3 & 1,83 & 0,00 & 0,0 & 10 & 82,71 & 2,87 & 0,00 & 0,0 & 9 & 72,72 \\
\hline 107 & 81,6 & 1,83 & 0,00 & 0,0 & 10 & 71,47 & 3,05 & 0,00 & 0,0 & 12 & 86,32 \\
\hline 108 & 57,0 & 1,65 & 0,00 & 0,0 & 0 & 44,24 & 3,66 & 0,00 & 0,0 & 10 & 79,99 \\
\hline 109 & 75,6 & 0,00 & 0,00 & 0,0 & 4 & 54,53 & 0,00 & 0,00 & 0,0 & 6 & 52,62 \\
\hline 110 & 69,4 & 5,49 & 1,22 & 22,2 & 9 & 81,64 & 5,30 & 2,13 & 40,2 & 12 & 76,04 \\
\hline 111 & 70,2 & 2,74 & 0,00 & 0,0 & 7 & 63,18 & 2,13 & 0,00 & 0,0 & 2 & 51,49 \\
\hline 112 & 83,7 & 1,83 & 0,00 & 0,0 & 9 & 67,81 & 1,83 & 0,00 & 0,0 & 5 & 52,06 \\
\hline 113 & 55,4 & 3,66 & 0,00 & 0,0 & 10 & 91,17 & 2,87 & 0,00 & 0,0 & 3 & 50,26 \\
\hline 114 & 49,0 & 2,44 & 0,00 & 0,0 & 9 & 74,26 & 2,26 & 0,00 & 0,0 & 9 & 73,45 \\
\hline 115 & 48,9 & 4,09 & 1,22 & 29,8 & 12 & 79,99 & 2,74 & 0,00 & 0,0 & 12 & 80,17 \\
\hline 116 & 69,4 & 1,83 & 1,65 & 90,2 & 10 & 75,09 & 1,83 & 1,22 & 66,7 & 9 & 78,88 \\
\hline 117 & 48,6 & 5,18 & 1,65 & 31,9 & 12 & 110,37 & 4,27 & 0,73 & 17,1 & 12 & 104,14 \\
\hline 118 & 56,8 & 1,95 & 0,00 & 0,0 & 9 & 71,62 & 4,09 & 0,00 & 0,0 & 8 & 72,22 \\
\hline 119 & 73,1 & 3,35 & 0,00 & 0,0 & 10 & 72,32 & 4,27 & 0,00 & 0,0 & 10 & 79,82 \\
\hline MW & 65,9 & 2,14 & 0,30 & 9,2 & 9,2 & 76,65 & 2,75 & 0,26 & 7,6 & 9,2 & 75,64 \\
\hline SD & 11,0 & 1,66 & 0,61 & 22,3 & 2,9 & 14,91 & 1,24 & 0,58 & 17,8 & 3,0 & 14,94 \\
\hline Max & 83,7 & 5,49 & 1,65 & 90,2 & 12,0 & 110,37 & 5,30 & 2,13 & 66,7 & 12,0 & 104,14 \\
\hline Min & 48,6 & 0,00 & 0,00 & 0,0 & 0,0 & 44,24 & 0,00 & 0,00 & 0,0 & 2,0 & 50,26 \\
\hline SW & 35,1 & 5,49 & 1,65 & 90,2 & 12,0 & 66,13 & 5,30 & 2,13 & 66,7 & 10,0 & 53,88 \\
\hline
\end{tabular}


Anhang

Tabelle 10: P-ERG- und OCT-Ergebnisse der Gruppe IV (Verdachtspatienten)

a) Eher Glaukom-manifest

\begin{tabular}{|c|c|c|c|c|c|c|c|c|c|c|c|}
\hline ID & Alter & P-ERG $[\mu \mathrm{V}]$ & B-P-ERG $[\mu \mathrm{V}]$ & B-weiß [\%] & OCT & $\varnothing \mathrm{RNFL}[\mu \mathrm{m}]$ & P-ERG $[\mu V]$ & B-P-ERG $[\mu \mathrm{V}]$ & B-weiß [\%] & OCT & $\varnothing \mathrm{RNFL}[\mu \mathrm{m}]$ \\
\hline & & \multicolumn{5}{|c|}{ OD } & \multicolumn{5}{|c|}{ OS } \\
\hline 200 & 70,7 & 3,66 & 0,00 & 0 & 9 & 91,38 & 2,44 & 1,22 & 50 & 12 & 108,07 \\
\hline 201 & 23,9 & 5,00 & 2,44 & 48,8 & 9 & 83,15 & 5,18 & 2,44 & 47,1 & 10 & 83,60 \\
\hline 202 & 18,9 & 5,91 & 2,74 & 46,4 & 9 & 76,54 & 4,88 & 2,44 & 50,0 & 9 & 80,65 \\
\hline 203 & 25,9 & 4,88 & 3,66 & 75,0 & 12 & 108,06 & 4,27 & 3,48 & 81,5 & 12 & 98,52 \\
\hline 204 & 69,4 & 4,88 & 0,00 & 0,0 & 8 & 83,50 & 3,05 & 0,00 & 0,0 & 9 & 85,25 \\
\hline 205 & 32,8 & 3,66 & 1,52 & 41,5 & 12 & 101,67 & 3,35 & 1,83 & 54,6 & 12 & 100,70 \\
\hline 206 & 37,4 & 1,04 & 0,00 & 0,0 & 11 & 81,83 & 1,22 & 0,00 & 0,0 & 3 & 56,69 \\
\hline 207 & 67,7 & 5,49 & 0,00 & 0,0 & 11 & 75,34 & 4,27 & 1,22 & 28,6 & 8 & 68,08 \\
\hline 208 & 18,0 & 4,88 & 2,44 & 50,0 & 11 & 92,57 & 4,88 & 2,13 & 43,6 & 10 & 94,14 \\
\hline 209 & 58,5 & 3,35 & 0,00 & 0,0 & 12 & 102,12 & 4,27 & 0,00 & 0,0 & 12 & 99,08 \\
\hline 210 & 45,2 & 10,37 & 1,83 & 17,6 & 12 & 98,50 & 9,15 & 3,05 & 33,3 & 12 & 99,25 \\
\hline 211 & 31,6 & 4,27 & 1,83 & 42,9 & 9 & 79,36 & 4,27 & 2,44 & 57,1 & 7 & 72,34 \\
\hline 212 & 49,4 & 3,05 & 0,73 & 23,9 & 12 & 100,45 & 3,66 & 1,22 & 33,3 & 11 & 91,41 \\
\hline
\end{tabular}

\begin{tabular}{|l|c|c|c|c|c|c|c|c|c|c|c|}
\hline MW & 42,2 & 4,65 & 1,32 & 26,6 & 10,5 & 90,34 & 4,22 & 1,65 & 36,9 & 9,8 & 87,52 \\
\hline SD & 19,3 & 2,13 & 1,28 & 25,6 & 1,5 & 11,04 & 1,84 & 1,16 & 24,8 & 2,7 & 14,93 \\
\hline Max & 70,7 & 10,37 & 3,66 & 75,0 & 12,0 & 108,06 & 9,15 & 3,48 & 81,5 & 12,0 & 108,07 \\
\hline Min & 18,0 & 1,04 & 0,00 & 0,0 & 8,0 & 75,34 & 1,22 & 0,00 & 0,0 & 3,0 & 56,69 \\
\hline SW & 52,7 & 9,33 & 3,66 & 75,0 & 4,0 & 32,72 & 7,93 & 3,48 & 81,5 & 9,0 & 51,38 \\
\hline
\end{tabular}


Anhang

b) Eher kein Glaukom

\begin{tabular}{|c|c|c|c|c|c|c|c|c|c|c|c|}
\hline ID & Alter & P-ERG $[\mu \mathrm{V}]$ & B-P-ERG $[\mu \mathrm{V}]$ & B-weiß [\%] & OCT & $\varnothing \mathrm{RNFL}[\mu \mathrm{m}]$ & P-ERG $[\mu \mathrm{V}]$ & B-P-ERG $[\mu \mathrm{V}]$ & B-weiß [\%] & OCT & $\varnothing \mathrm{RNFL}[\mu \mathrm{m}]$ \\
\hline & & \multicolumn{5}{|c|}{ OD } & \multicolumn{5}{|c|}{ OS } \\
\hline 213 & 29,1 & 5,00 & 3,05 & 61,0 & 10 & 89,75 & 4,70 & 1,22 & 26,0 & 11 & $\overline{97,46}$ \\
\hline 214 & 66,8 & 1,83 & 0,00 & 0,0 & 12 & 112,66 & 0,00 & 0,00 & 0,0 & 11 & 96,32 \\
\hline 215 & 69,6 & 1,83 & 0,00 & 0,0 & 12 & 100,10 & 2,44 & 0,00 & 0,0 & 12 & 91,81 \\
\hline 216 & 45,9 & 7,13 & 3,66 & 51,3 & 12 & 93,06 & 5,91 & 3,05 & 51,6 & 12 & 88,41 \\
\hline 217 & 21,8 & 3,05 & 2,74 & 89,8 & 12 & 97,50 & 3,05 & 2,13 & 69,8 & 12 & 97,75 \\
\hline 218 & 37,8 & 3,96 & 2,13 & 53,8 & 12 & 105,50 & 5,91 & 2,13 & 36,0 & 12 & 105,50 \\
\hline 219 & 40,3 & 5,30 & 2,56 & 48,3 & 8 & 87,97 & 5,18 & 2,44 & 47,1 & 10 & 90,41 \\
\hline MW & 44,5 & 4,01 & 2,02 & 43,5 & 11,1 & 98,08 & 3,88 & 1,57 & 32,9 & 11,4 & 95,38 \\
\hline SD & 18,0 & 1,95 & 1,46 & 32,7 & 1,6 & 8,84 & 2,18 & 1,20 & 26,3 & 0,8 & 5,76 \\
\hline Max & 69,6 & 7,13 & 3,66 & 89,8 & 12,0 & 112,66 & 5,91 & 3,05 & 69,8 & 12,0 & 105,50 \\
\hline Min & 21,8 & 1,83 & 0,00 & 0,0 & 8,0 & 87,97 & 0,00 & 0,00 & 0,0 & 10,0 & 88,41 \\
\hline SW & 47,8 & 5,30 & 3,66 & 89,8 & 4,0 & 24,69 & 5,91 & 3,05 & 69,8 & 2,0 & 17,09 \\
\hline
\end{tabular}




\section{Anhang}

Tabelle 16: Häufigkeitsverteilung der 72 gesunden Studienteilnehmer gemäß RNFL-Messung

\begin{tabular}{|l|c|c|c|c|}
\cline { 2 - 5 } \multicolumn{1}{c|}{} & $\mathrm{n}=$ & $\%$ & Ausgang & $\begin{array}{c}\text { Übereinstimmung } \\
{[\%]}\end{array}$ \\
\hline I) Augen gesunder Probanden & 44 & 61,1 & 44 & 100,0 \\
\hline II) Augen von Glaukompatienten & 5 & 6,9 & 10 & 50,0 \\
\hline III) Augen von Verdachtspatienten & & & & \\
a) "eher Glaukom manifest" & 13 & 18,1 & 18 & 27,8 \\
b) "eher kein Glaukom" & 10 & 13,9 & 10 & 100,0 \\
\hline Gesamt & 72 & 100,0 & 82 & 78,0 \\
\hline
\end{tabular}

Tabelle 17: Häufigkeitsverteilung der 10 erkrankten Studienteilnehmer gemäß RNFL-Messung

\begin{tabular}{|l|c|c|c|c|}
\cline { 2 - 5 } \multicolumn{1}{c|}{} & $\mathrm{n}=$ & $\%$ & Ausgang & $\begin{array}{c}\text { Übereinstimmung } \\
{[\%]}\end{array}$ \\
\hline I) Augen gesunder Probanden & 0 & 0 & 44 & 100,0 \\
\hline II) Augen von Glaukompatienten & 5 & 50,0 & 10 & 50,0 \\
\hline III) Augen von Verdachtspatienten & & & & \\
a) "eher Glaukom manifest" & 5 & 50,0 & 18 & 27,8 \\
b) "eher kein Glaukom" & 0 & 0 & 10 & 100,0 \\
\hline Gesamt & 10 & 100,0 & 82 & 78,0 \\
\hline
\end{tabular}

Tabelle 18: Häufigkeitsverteilung der 66 gesunden Studienteilnehmer gemäß beiden Messungen

\begin{tabular}{|l|c|c|c|c|}
\cline { 2 - 5 } \multicolumn{1}{c|}{} & $\mathrm{n}=$ & $\%$ & Ausgang & $\begin{array}{c}\text { Übereinstimmung } \\
{[\%]}\end{array}$ \\
\hline I) Augen gesunder Probanden & 43 & 65,2 & 44 & 97,7 \\
\hline II) Augen von Glaukompatienten & 2 & 3,0 & 10 & 80,0 \\
\hline III) Augen von Verdachtspatienten & & & & \\
a) "eher Glaukom manifest" & 12 & 18,2 & 18 & 33,3 \\
b) "eher kein Glaukom" & 9 & 13,6 & 10 & 90,0 \\
\hline Gesamt & 66 & 100,0 & 82 & 80,5 \\
\hline
\end{tabular}




\section{Anhang}

Tabelle 19: Häufigkeitsverteilung der 71 gesunden Studienteilnehmer gemäß BWERG-Messung

\begin{tabular}{|l|c|c|c|c|}
\cline { 2 - 5 } \multicolumn{1}{c|}{} & $\mathrm{n}=$ & $\%$ & Ausgang & $\begin{array}{c}\text { Übereinstimmung } \\
{[\%]}\end{array}$ \\
\hline I) Augen gesunder Probanden & 43 & 60,6 & 44 & 97,7 \\
\hline II) Augen von Glaukompatienten & 2 & 4,2 & 10 & 11,1 \\
\hline III) Augen von Verdachtspatienten & & & & \\
a) "eher Glaukom manifest" & 16 & 22,5 & 18 & 33,3 \\
b) "eher kein Glaukom" & 9 & 12,7 & 10 & 90,0 \\
\hline Gesamt & 71 & 100,0 & 82 & 75,6 \\
\hline
\end{tabular}

Tabelle 20: Häufigkeitsverteilung der 11 erkrankten Studienteilnehmer gemäß BWERG-Messung

\begin{tabular}{|l|c|c|c|c|}
\cline { 2 - 5 } \multicolumn{1}{c|}{} & $\mathrm{n}=$ & $\%$ & Ausgang & $\begin{array}{c}\text { Übereinstimmung } \\
{[\%]}\end{array}$ \\
\hline I) Augen gesunder Probanden & 1 & 9,1 & 44 & 97,7 \\
\hline II) Augen von Glaukompatienten & 7 & 63,6 & 10 & 70,0 \\
\hline III) Augen von Verdachtspatienten & & & & \\
a) "eher Glaukom manifest" & 2 & 18,2 & 18 & 11,1 \\
b) "eher kein Glaukom" & 1 & 9,1 & 10 & 90,0 \\
\hline Gesamt & 11 & 100,0 & 82 & 74,4 \\
\hline
\end{tabular}

Tabelle 21: Häufigkeitsverteilung der 5 erkrankten Studienteilnehmer gemäß beiden Messungen

\begin{tabular}{|l|c|c|c|c|}
\cline { 2 - 5 } \multicolumn{1}{c|}{} & $\mathrm{n}=$ & $\%$ & Ausgang & $\begin{array}{c}\text { Übereinstimmung } \\
{[\%]}\end{array}$ \\
\hline I) Augen gesunder Probanden & 0 & 0 & 44 & 100,0 \\
\hline II) Augen von Glaukompatienten & 4 & 80,0 & 10 & 40,0 \\
\hline III) Augen von Verdachtspatienten & & & & 5,6 \\
a) "eher Glaukom manifest" & 1 & 20,0 & 18 & 100,0 \\
b) "eher kein Glaukom" & 0 & 0 & 10 & 100,0 \\
\hline Gesamt & 5 & 100,0 & 82 & \\
\hline
\end{tabular}




\section{Danksagung}

Herrn Prof. Dr. J. Roider danke ich für die zur Verfügung gestellten Untersuchungsmaterialien und -räume sowie die Möglichkeit der Realisierung dieser Arbeit an der Augenklinik des Universitätsklinikums Kiel.

Das Thema wurde mir freundlicherweise von Herrn Dr. C. Kandzia gestellt. Ihm danke ich sehr herzlich für die intensive Unterstützung in der praktischen Umsetzung der Arbeit sowie in der Begleitung der schriftlichen Fertigstellung der Dissertation.

Ein großer Dank gilt auch den Krankenschwestern und Ärzten der Augenklinik, die mich die Untersuchungstechniken lehrten und mir die Gelegenheit zur Durchführung der Arbeit gaben. Insbesondere sei Herrn Dr. F. Treumer gedankt, der alle Probanden mituntersuchte und mich jederzeit freundlich und hilfsbereit unterstützte.

Ebenfalls möchte ich mich bei Herrn Dipl. math. Jochens, dem Statistiker, für die stets unkomplizierte und engagierte Zusammenarbeit bedanken.

Zuletzt danke ich meiner Familie und meinen Freunden von Herzen, dass sie mir das Studium ermöglichten, mich begleiteten und fortwährend motivierten, meinen Weg weiter zu gehen.

Rostock, Februar 2013

Katrin Wolff 


\section{Lebenslauf}

Name, Vorname:

Geburtsdatum/-ort:

Familienstand:

Eltern:

Veröffentlichung

$12 / 12$

\section{Berufstätig} seit $01 / 11$

\section{Schulbildung}

08/91 - 08/95

$09 / 95-06 / 04$

\section{Hochschulausbildung}

10/04 - 10/10

\section{Praktisches Jahr}

$04 / 10-08 / 10$

$12 / 09-04 / 10$

$08 / 09-12 / 09$

\section{Famulaturen}

03/09-04/09

$09 / 08$

08/08

02/08-03/08

$07 / 07-09 / 07$

\section{Pflegepraktika}

09/05

08/05

07/04
Wolff, Katrin

17.03.1985 / 17166 Teterow

ledig

Hubert Wolff

Rosemarie Wolff, geb. Hennek

Wolff K, Treumer F, Jochens A, Roider JB, Kandzia C.

[Screening for Glaucoma by Blue Light-Pattern ERG and OCT - Are the Methods Comparable?]. Klin Monbl Augenheilkd; 229: 1215-1222

Assistenzärztin in der Universitätsaugenklinik Rostock

Grundschule Matgendorf

Europaschule Gymnasium Teterow

Humanmedizin an der Christian-Albrechts-Universität zu Kiel

Chirurgie - Asklepios Westklinikum Hamburg

Ophthalmologie/Orth- und Pleoptik - UKSH, Campus Kiel

Innere Medizin - Friedrich-Ebert-Krankenhaus Neumünster

Kinderchirurgie - Hôpital Sainte-Justine (Montréal)

Allgemeinarztpraxis - B. Schmidt (Kiel)

Innere Medizin - Städtisches Klinikum München-Schwabing

Augenklinik Bellevue/Augenärzte im „Wilhelminenhaus“ Kiel

Ophthalmologie/Orth- u. Pleoptik - UKSH, Campus Kiel

Kinderkardiologie - Universitätsklinikum Rostock

Neurologie - Bundeswehrkrankenhaus Hamburg

Augenheilkunde - UKSH, Campus Lübeck 